SERVIÇO DE PÓS-GRADUAÇÃO DO ICMC-USP Data de Depósito: 17/10/2005

Assinatura:

\title{
Equivalências de contato topológica e bi-Lipschitz de germes de aplicações diferenciáveis ${ }^{1}$
}

\author{
João Carlos Ferreira Costa
}

Orientadora: Profa. Dra. Maria Aparecida Soares Ruas

Tese apresentada ao Instituto de Ciências Matemáticas e de Computação - ICMC-USP, como parte dos requisitos para obtenção do título de Doutor em Ciências - Matemática.

USP - São Carlos

Outubro de 2005

${ }^{1}$ Trabalho realizado com o auxílio financeiro da FAPESP processo: 01/14577-0 
Aluno: João Carlos Ferreira Costa

A Comissão Julgadora:

Profa. Dra. Maria Aparecida Soares Ruas

Prof. Dr. Lev Birbrair

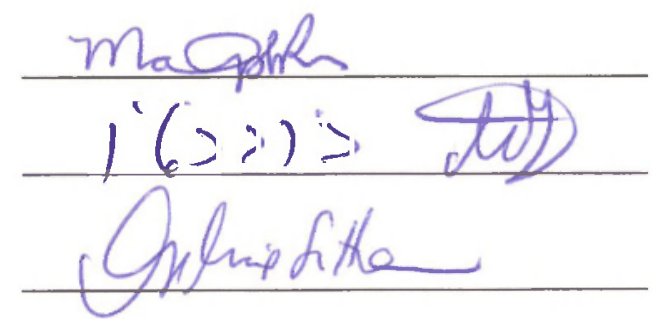

Profa. Dra. Angela Maria Sitta

Prof. Dr. Daniel Cantergiani Panazzolo

Prof. Dr. João Nivaldo Tomazella

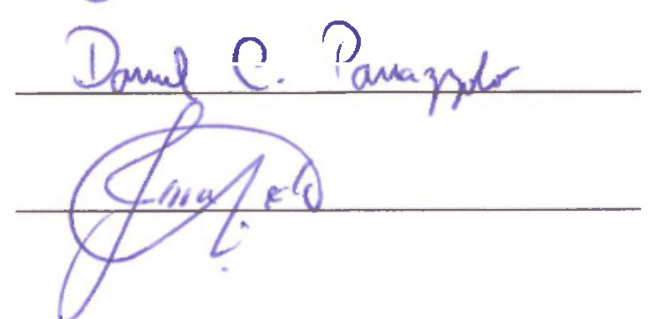


À Sabrina, minha esposa 


\section{Agradecimentos}

Agradeço a Deus pelo dom da vida e pela possibilidade de realizar mais este sonho. À Mãe do cón, por sempre interceder por este seu filho.

À Profa. Dra. Maria Aparecida Soares Ruas, Cidinha, por toda dedicaçã̃o, paciência e amizade durante a sua valiosa orientação. Gostaria de registrar minha admiração por sua imensa responsabilidade, empenho, liderança, carinho e respeito, pelo nosso grupo de Singularidades. Muito obrigado!

Ao Prof. Dr. Lev Birbrair e ao Prof. Dr. Alexandre Fernandes, amigos e colaboradores deste trabalho, agradeço pelas valiosas sugestões, e pela oportunidade de me apresentarem as praias de Fortaleza.

A Profa. Dra. Angela Maria Sitta, por ter me cncaminhado com maestria para o doutorado.

À minha esposa Sabrina, minha Sá, pela paciência, amor e dedicação, nestes momentos em que estive ausente.

Aos meus pais, João e Cidinha, meus primeiros mestres, por todo incentivo e conliança na caminhada até aqui.

$\grave{\Lambda}$ minha irmã, Fernanda, uma pessoa tão especial $\mathrm{em}$ minha vida, por todo seu apoio e torcida.

A todos os meus familiares e amigos, que participaram da minha vida durante estes anos de doutoramento.

Aos amigos da minha turma de doutorado: Lizandro, Elenice, Elíris, Karina, Esdras, Zé Paulo, Roland, Maurício e Silas, pelo companheirismo e momentos de descontração.

A todos os amigos da pós-graduação, da república, do futebol, da minha salinha de estudos e do laboratório de Singularidades, pelo agradável convívio.

Aos professores e amigos do ICMC, pelas conversas, sugestões e amizade.

Aos funcionários do ICMC, pela eficiência e disposição em todos os momentos.

A FAPESP pelo apoio financeiro para a realização deste trabalho. 
..."Leva-me aonde os homens,

Necessitem Tuas palavras,

Necessitem, Tua força de viver

Onde falte a esperança,

Onde tudo seja triste, simplesmente,

Por não saber de Ti."... 


\section{Resumo}

Neste trabalho estudamos a equivalência de contato nas versões topológica e biI.ipschitz.

Para a equivalência de contato topológica (ou $\mathcal{C}^{0}-\mathcal{K}$-equivalência) caracterizamos completamente os germes de funções reais com o invariante chamado função tenda. Além disso, apresentamos uma forma normal para os germes de funções analíticas reais $\mathcal{C}^{0}-\mathcal{K}$ linitas quando a dimensão da fonte é $n=2$. Para germes de aplicações $\left(\mathbb{R}^{n}, 0\right) \rightarrow\left(\mathbb{R}^{p}, 0\right)$, se $n<p$, provamos que todos os germes $\mathcal{C}^{0}-\mathcal{K}$-finitos são $\mathcal{C}^{0}-\mathcal{K}$-equivalentes. Se $n \geq p$, nossos principais resultados são para famílias de germes de aplicações. Com hipóteses de regularidade para a família dos conjuntos dos zeros, obtemos condições suficientes para a $\mathcal{C}^{0}-\mathcal{K}$-trivialidade de famílias de germes $\mathcal{C}^{0}$ - $\mathcal{K}$-finitos. No caso particular de curvas, quando $p=n-1$, mostramos algumas situações em que o número de semi-ramos da curva é um invariante completo para a $\mathcal{C}^{0}$ - $\mathcal{K}$-equivalência.

Introduzimos o conceito de $\mathcal{K}$-bi-Lipschitz equivalência e restringimos este estudo para o caso de funçôes. O principal resultado mostra que o número de classes de $\mathcal{K}$-bi-Lipschitz equivalência dos germes de funçōes polinomiais é finito. 


\begin{abstract}
In this work we study the contact equivalence from the topological and bi-Lipschitz point of view.

We characterize completely the real function-germs with respect to $\mathcal{C}^{0}-\mathcal{K}$-equivalence, defining an invariant called tent function. Furthermore, we present a normal form for $\mathcal{C}^{0}-\mathcal{K}$-finitely determined real analytic function-germs when the source dimension is $n=2$. For map-germs $\left(\mathbb{R}^{n}, 0\right) \rightarrow\left(\mathbb{R}^{p}, 0\right)$, if $n<p$, we prove that all $\mathcal{C}^{0}-\mathcal{K}$-finite germs are $\mathcal{C}^{0}-\mathcal{K}$ equivalent. If $n \geq p$, our main results arc related to families of germs. Based upon regularity conditions on the families of zero-sets, we give sufficient conditions for the $\mathcal{C}^{0}-\mathcal{K}$ triviality of families of $\mathcal{C}^{0}-\mathcal{K}$-finite germs. In the special case of curves $(p=n-1)$, we prove in some cases that the number of half-branches of the curve is a complete invariant for the $\mathcal{C}^{0}$ - $\mathcal{K}$-equivalence.

We introduce the definition of $\mathcal{K}$-bi-Lipschitz equivalence and we study this equivalence relation for functions. Our main result shows that the number of $\mathcal{K}$-bi-Lipschit\% cquivalence classes of polynomial function-germs is finite.
\end{abstract}




\section{Sumário}

Introdução 1

1 Preliminares 4

1.1 Conjuntos algébricos e semialgébricos . . . . . . . . . . 4

1.2 Estratificações e triangulações . . . . . . . . . . . . . . . . . 6

1.3 Conjuntos analíticos, semianalíticos e subanalíticos . . . . . . . . 11

1.4 Estruturas o-minimais . . . . . . . . . . . . . . . . . 12

1.5 Resultados da teoria de singularidades $\ldots \ldots \ldots \ldots \ldots$

2 Equivalência de contato topológica $\left(\mathcal{C}^{0}-\mathcal{K}\right.$-equivalência) 21

2.1 Resultados de Nishimura e o caso $n=p \ldots \ldots \ldots \ldots \ldots . \ldots \ldots$

2.2 Outras propriedades da $\mathcal{C}^{0}-\mathcal{K}$-equivalência $\ldots \ldots \ldots \ldots \ldots$

2.3 Interpretação geométrica . . . . . . . . . . . . . . . . 35

$\begin{array}{llll}3 & \mathcal{C}^{0} \text { - } \mathcal{K} \text {-equivalência de aplicações } & \text { Caso } n<p & 37\end{array}$

$4 \mathcal{C}^{0}$-K-equivalência de funçōes $\quad 41$

4.1 Funções tenda . . . . . . . . . . . . . . . . . . . 42

4.2 Polinômios de 2 variávcis e $\mathcal{K}$-invariantes . . . . . . . . . . . . . 45

4.3 Interpretação geométrica . . . . . . . . . . . . . . . . . . 49

$5 \quad \mathcal{C}^{0}-\mathcal{K}$-equivalência de aplicações $\quad$ Caso $n \geq p \quad 53$

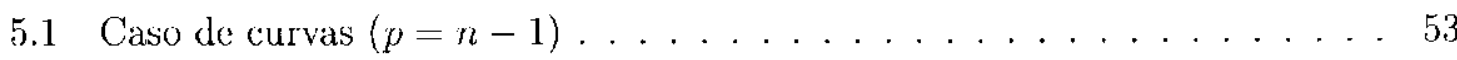

5.2 Famílias de germes de aplicações . . . . . . . . . . . . . . . 57

6 Equivalência de contato bi-Lipschitz ( $\mathcal{K}$-bi-Lipschitz equivalência) $\quad 68$

6.1 Teorema de finitude . . . . . . . . . . . . . . . . . . 76

6.2 Resultados adicionais . . . . . . . . . . . . . . . 77

6.3 Outros invariantes da $\mathcal{K}$-bi-Lipschitz equivalênncia $\ldots \ldots \ldots \ldots 78$

$\begin{array}{lc}\text { Comentários finais } & 80\end{array}$

Referências Bibliográficas $\quad 82$ 


\section{Introdução}

A noção de equivalência de contato, ou $\mathcal{K}$-equivalência, foi introduzida por J. Mather para reduzir o problema da classificação $C^{\infty}$ dos germes de aplicações estáveis para o problema da classificação de $\mathbb{R}$-álgebras (ver [33|, |34|). Esta relação de equivalência foi um importante passo no estudo da $\mathcal{A}$-equivalência destes germes. Na literatura clássica da teoria de singularidades são bem conhecidas as propriedades, invariantes e caracterizaçoes da $\mathcal{K}$-equivalência. Por exemplo, dados dois germes de aplicações $f, g:\left(\mathbb{R}^{n}, 0\right) \rightarrow\left(\mathbb{R}^{p}, 0\right)$, Mather [33] mostrou que a $\mathcal{K}$-equivalência entre $f$ e $g$ pode ser determinada através do tipo de contato dos gráficos com o $\mathbb{R}^{n}$ na origem. Isto é, $f$ e $g$ são $\mathcal{K}$-equivalentes se, e somente se, existe un $\mathcal{C}^{\infty}$-difeomorfismo $H:\left(\mathbb{R}^{n} \times \mathbb{R}^{p}, 0\right) \rightarrow\left(\mathbb{R}^{n} \times \mathbb{R}^{p}, 0\right)$ que deixa $\mathbb{R}^{n} \times\{0\}$ invariante e $I(\operatorname{graf}(f))=\operatorname{graf}(g)$. Este resultado foi depois estendido por J. Montaldi. De fato, Montaldi [39] introduz uma definição de contato puramente geomćtrica: dois pares de subvariedades do $\mathbb{R}^{n},\left(\mathcal{X}_{1}, \mathcal{Y}_{1}\right)$ e $\left(\mathcal{X}_{2}, \mathcal{Y}_{2}\right)$, têm o mesmo tipo de contato na origem se existe um $\mathcal{C}^{\infty}$-difeomorfismo $H: \mathbb{R}^{n} \rightarrow \mathbb{R}^{n}$ tal que $H\left(\mathcal{X}_{1}\right)=\mathcal{X}_{2}$ e $H\left(\mathcal{Y}_{1}\right)=\mathcal{Y}_{2}$. Ele provou que esta definição de contato entre subvariedades ć equivalente à definição de $\mathcal{K}$-equivalência para convenientes germes de aplicações.

No entanto, a classificação dos germes de aplicações módulo difcomorfismos apresenta muita rigidez. Então, é natural pensar em classificações de germes obtidas por meio de relações de equivalência mais fracas. Esta é uma das motivações deste trabalho, que tem por objetivo estudar propriedades e invariantes da equivalência de contato nas versões topológica (via homeomorfismos) e bi-Lipschitz (via homeomorfismos bi-Lipschitz). Para a $\mathcal{K}$-equivalência topológica, ou $\mathcal{C}^{0}-\mathcal{K}$-cquivalência, não existem critćrios ou caracterizações análogos aos da $\mathcal{K}$-equivalência de Mather. $O$ artigo de $T$. Nishimura [45] pode ser visto como o primeiro a considerar o problema de determinar critérios algébricos para a $\mathcal{C}^{0}-\mathcal{K}$ equivalência. Para o caso $n=p$, Nishimura [45] apresenta o único invariante completo conhecido para a $\mathcal{C}^{0}$ - $\mathcal{K}$-equivalência: o valor absoluto do grau. Esta mesma carência de referencias e resultados ocorre também para a $\mathcal{K}$-bi-Lipschitz equivalência. Acreditamos que este trabalho é o primeiro a introduzir o estudo da $\mathcal{K}$-bi-Lipschitz equivalência. Assim, 
procuramos neste trabalho estender as idéias de Nishimura no caso da $\mathcal{C}^{(0}-\mathcal{K}$-equivalôncia e investigar o que ocorre com a $\mathcal{K}$-bi-Lipschitz equivalência, sobretudo no caso de germes de funções reais.

No caso da $\mathcal{C}^{0}-\mathcal{K}$-equivalência, caracterizamos por completo os germes de funções reais com o invariante chamado função tenda (Teorema 4.3). Além disso, apresentamos uma forma normal para os germes de funçõos analíticas reais $\mathcal{C}^{0}-\mathcal{K}$-finitas quando $n=2$ (Teorema 4.8). Para germes de aplicaçốes, quando $n<p$ provamos que todos os germes $\mathcal{C}^{0}-\mathcal{K}$-finitos são equivalcntes (Teorema 3.6). Quando $n>p$, destacamos em nosso estudo o caso de famílias de germes de aplicações. Com hipóteses de regularidade para a família dos conjuntos dos zeros, apresentamos condições suficientes para a $\mathcal{C}^{0}-\mathcal{K}$-trivialidade das famílias de germes $\mathcal{C}^{0}-\mathcal{K}$-finitos (Teorema 5.20 e Teorema 5.12). No caso particular de curvas, $p=n-1$, mostramos algumnas situações em que o número de semi-ramos da curva é umn invariante completo da $\mathcal{C}^{0}-\mathcal{K}$-equivalência.

Para a $\mathcal{K}$-bi-Iipschitz equivalência, o principal resultado (Teorema 6.14) estabelece que existe um número finito de tipos $\mathcal{K}$-bi-Lipschitz dos germes de funções polinomiais reais. Responder sobre a finitude dos tipos $\mathcal{K}$-bi-Lipschitz dos germes de funções polinomiais é urra questão central neste estudo, pois a $\mathcal{K}$-bi-Lipschitz equivalência é intermediária entre a $\mathcal{R}$-bi-Lipschitz equivalência e a equivalência bi-Lipschitz dos conjuntos dos zeros e sabemos que para estas duas últimas relações de equivalência a questão sobre finitude está respondida. De fato, J.-P. Henry e A. Parusinski [25] mostraram que a equivalência bi-Lipschitz (ou $\mathcal{R}$-bi-Lipschitz equivalência) dos germes de funções analíticas reais tem moduli enquanto T. Mostowski e o próprio Parusinski (ver [41],[48],[47]) mostraram que os tipos bi-Lipschitz dos germes de variedade reais analíticas não têm moduli.

Além disso, de maneira análoga às idéias de Mather-Montaldi, procuramos estabelecer uma caracterização geométrica para ambas relações de equivalência (Teorema 4.10 e T'eorema 6.13, respectivamente).

O trabalho possui seis capítulos. O Capítulo 1 está dividido em duas partes. A primeira, define as categorias dos conjuntos que aparecem no texto, cita algumas de suas propriedades e apresenta resultados relacionados às tcorias de estratificação e triangulação. A segunda, refere-se aos resultados básicos da teoria de singularidades relacionados à $\mathcal{K}$-equivalência dos germes de aplicações diferenciáveis.

No Capítulo 2 definimos a $\mathcal{C}^{0}-\mathcal{K}$-equivalência, tratamos da questão da $\mathcal{C}^{0}-\mathcal{K}$-determinação finita e apresentamos os resultados de Nishimura [45]. Dentre estes resultados destacamos o Lema 2.14 que é uma das principais ferramentas para os capítulos seguintes. No final da Seção 2.1, completamos o estudo da $\mathcal{C}^{0}-\mathcal{K}$-equivalência para $n=p$, com o 
Teorema 2.20 e a Proposição 2.21.

O Capítulo 3 trata da $\mathcal{C}^{0}-\mathcal{K}$-equivalência dos germes de aplicações $\left(\mathbb{R}^{n}, 0\right) \rightarrow\left(\mathbb{R}^{p}, 0\right)$, com $n<p$. Neste caso, mostramos que a $\mathcal{K}$-equivalência topológica não distingue germes $\mathcal{C}^{0}-\mathcal{K}$-finitos. Mais precisamente, provamos que quaisquer dois germes $C^{0}-\mathcal{K}$-finitamente determinados $f, g:\left(\mathbb{R}^{n}, 0\right) \rightarrow\left(\mathbb{R}^{p}, 0\right), n<p$, são $C^{0}-\mathcal{K}$-equivalentes.

O Capítulo 4 é dedicado à $\mathcal{C}^{0}-\mathcal{K}$-equivalência dos germes de funções. Apresentamos um invariante completo, que chamamos de função tenda. Em particular, no caso de germes de funções analíticas com $n=2$, o invariante é muito simples: uma sequência finita de elementos iguais a 1 ou -1 . Provamos ainda que todos estes invariantes admitem uma realização polinomial e estabelecemos urna forma normal para os germes de funções analíticas $C^{0}-\mathcal{K}$-finitamente determinadas. Estes resultados encontram-se em [1]. Na última seção do capítulo, apresentamos uma interpretação geométrica para a $\mathcal{C}^{0}$ - $\mathcal{K}$-equivalência.

Xo Capítulo 5 estudamos a $\mathcal{C}^{0}-\mathcal{K}$-equivalência dos germes $\left(\mathbb{R}^{n}, 0\right) \rightarrow\left(\mathbb{R}^{p}, 0\right)$, para $n \geq p$. Procuramos destacar o que acontece quando $p \neq 1$, pois os germes de funções são tratados separadamente no Capítulo 4. No caso $n>p \neq 1$, nossos resultados ainda são incompletos. Em particular, nos conceritramos no que acontece quando $p=n-1, n>2$. Este caso o de especial interesse pois os germes de aplicações $f:\left(\mathbb{R}^{n}, 0\right) \rightarrow\left(\mathbb{R}^{n-1}, 0\right)$ definem germes de curvas, $X=f^{-1}(0)$, o que torna o estudo da $C^{0}-\mathcal{K}$-equivalência mais tratável. Em algumas situações, provamos que o número de semi-ramos de uma curva $X=f^{-1}(0)$ é um invariante completo da $C^{0}-\mathcal{K}$-equivalência. Alguns resultados deste capítulo são obtidos através de argumentos apresentados no Capítulo 4. A parte principal deste capítulo é a Seção 5.2, que trata de famílias de germes de aplicações. Para famílias, obtivemos resultados mais precisos na tentativa de caracterizar a $\mathcal{C}^{0}$-K -equivalência. Com hipóteses de regularidade para a família dos conjuntos dos zeros, apresentamos condições suficientes para a $\mathcal{C}^{0}-\mathcal{K}$-trivialidade de famílias de germes $\mathcal{C}^{0}-\mathcal{K}$-finitos.

Finalmente, no Capítulo 6 , introduzimos o estudo da $\mathcal{K}$-bi-Lipschitz equivalência. Mostramos um teorema de finitude para os tipos $\mathcal{K}$-bi-Lipschitz dos germes de funções polinomiais. Usando mais uma vez argumentos geométricos, definimos a $\mathcal{K}$ - $\mathcal{M}$-bi-Lipschitz cquivalência e provamos que esta nova relação de equivalência caracteriza a $\mathcal{K}$-bi-Lipschitz equivalência dos germes de funções. Os principais resultados do capítulo encontram-se err [9].

Terminamos este trabalho com alguns Comentários finais, logo após o Capítulo 6 . 


\section{Capítulo 1}

\section{Preliminares}

Na primeira parte deste capítulo definimos as seguintes categorias de conjuntos: algébricos, semialgébricos, analíticos, semianalíticos e subanalíticos. Em seguida, apresentamos uma breve introdução sobre estruturas o-minimais. Os resultados descritos nas Seções $1.1,1.2,1.3$ e 1.4 podem ser encontrados nas referências [29], [11], [5] e [14].

\subsection{Conjuntos algébricos e semialgébricos}

Definição 1.1. Um conjunto $X \subset \mathbb{R}^{n}$ é algébrico se existem funções polinomiais $f_{i}: \mathbb{R}^{n} \rightarrow \mathbb{R}, i=1, \ldots, k$ tais que $X=\left\{x \in \mathbb{R}^{n} \mid f_{1}(x)=\ldots=f_{k}(x)=0\right\}$.

Como nossos objetos são reais, poderíamos simplesmente dizer que $X$ é algébrico se existe uma função polinomial $f: \mathbb{R}^{n} \rightarrow \mathbb{R}$, tal que $X=\left\{x \in \mathbb{R}^{n} \mid f(x)=0\right\}$. Basta considerar $f=f_{1}^{2}+\ldots+\int_{k}^{2}$.

Exemplo 1.2. $\Lambda$ esfera padrão unitária $S^{n-1}=\left\{\left(x_{1}, \ldots, x_{n}\right) \in \mathbb{R}^{n} \mid x_{1}^{2}+\ldots+x_{n}^{2}=1\right\}$ é un conjunto algébrico. De fato, considerando $f\left(x_{1}, \ldots, x_{n}\right)=\sum_{i=1}^{n} x_{i}^{2}-1$, claramente $f^{-1}(0)=S^{n-1}$.

Proposição 1.3. Sejam $X, Y$ conjuntos algébricos em $\mathbb{R}^{n}$. Então, $X \cup Y$ e $X \cap Y$ são conjuntos algébricos.

Proposição 1.4. Sejam $Y \subset \mathbb{R}^{p}$ um conjunto algébrico e $F: \mathbb{R}^{n} \rightarrow \mathbb{R}^{p}$ uma uplicação polinomial. Entäo, $F^{-1}(Y) \subset \mathbb{R}^{n}$ é um conjunto algébrico.

A Proposição 1.4 nos induz a questionar se a imagem de um conjunto algébrico por uma aplicação polinomial é um conjunto algćbrico. Isso nem sempre ocorre. Observe o contra-exemplo a seguir. 
Exemplo 1.5. Considere o círculo padrão unitário $S^{1}$ em $\mathbb{R}^{2}$ e $\pi: \mathbb{R}^{2} \rightarrow \mathbb{R}$ a projeção canônica $\pi(x, y)=x$. Pelo Exemplo 1.2, $S^{1}$ é um conjunto algébrico, mas $\pi\left(S^{1}\right)=[-1,1]$ não é algébrico.

Este contra-cxcmplo motiva a definição de uma classe mais geral que a dos conjuntos algébricos, a saber a classe dos conjuntos semialgébricos.

Definição 1.6. Um conjunto $X \subset \mathbb{R}^{n}$ é semialgébrico básico se existem funções polinomiais $f, g_{1}, \ldots, g_{k}: \mathbb{R}^{n} \rightarrow \mathbb{R}$ tais que

$$
X=\left\{x \in \mathbb{R}^{n} \mid f(x)=0\right\} \cap\left(\bigcap_{i=1}^{k}\left\{x \in \mathbb{R}^{n} \mid g_{i}(x)>0\right\}\right) .
$$

Um conjunto semialgébrico é a reunião finita de conjuntos semialgébricos básicos. Logo, se $X \subset \mathbb{R}^{n}$ é semialgébrico, existem funções polinomiais $f_{i}, g_{i j}$ definidas em $\mathbb{R}^{n}$, tais que

$$
X=\bigcup_{i=1}^{k}\left\{x \in \mathbb{R}^{n} \mid f_{i}(x)=0\right\} \cap\left(\bigcap_{j=1}^{s_{i}}\left\{x \in \mathbb{R}^{n} \mid g_{i j}(x)>0\right\}\right) .
$$

\section{Observaçōes.}

a) Os conjuntos algébricos são, claramente, conjuntos semialgébricos.

b) O conjunto $X=\left\{(x, y) \in \mathbb{R}^{2} \mid x^{2}+y^{2} \leq 1\right\}$ é semialgébrico, mas não é algébrico.

c) Em $\mathbb{R}$, um conjunto semialgébrico é a reunião finita de intervalos abertos, fcchados, semiabertos e pontos.

d) Se $X, Y \subset \mathbb{R}^{n}$ são conjuntos semialgébricos, então $X \cup Y, X-Y, \mathbb{R}^{n}-X, \mathbb{R}^{n}-Y$ e $X \cap Y$ são também semialgébricos.

c) Se $X \subset \mathbb{R}^{n}$ e $Y \subset \mathbb{R}^{p}$ são conjuntos semialgébricos, então $X \times Y \subset \mathbb{R}^{n} \times \mathbb{R}^{p}$ é um conjunto semialgébrico.

Proposição 1.7. Se $X \subset \mathbb{R}^{n}$ é um conjunto semialyébrico, então o fecho de $X$, denotado por $\bar{X}$, é um conjunto semialgébrico.

Definição 1.8. Seja $X \subset \mathbb{R}^{n}$. Dizemos que a função $F: X \rightarrow \mathbb{R}$ é semialgébrica se o gráfico de $F, \operatorname{graf}(F)=\{(x, y) \in X \times \mathbb{R} \mid y=F(x)\}$, é um conjunto semialgébrico.

A proposição a seguir decorre de um teorema estrutural para conjuntos semialgébricos (ver Teorema da Decomposição Cilíndrica [5]). 
Proposição 1.9. Se $X \subset \mathbb{R}^{n}$ é um conjunto semialgébrico, então $X$ tem um número finito de componentes conexas e cada uma destas componentes é ainda um conjunto semialgébrico.

Teorema 1.10. (Tarski-Seidenberg (5)) A imagem de um conjunto semialgébrico $X \subset \mathbb{R}^{n}$ por uma projeção $\pi: \mathbb{R}^{n} \rightarrow \mathbb{R}^{n-1}$ é um conjunto semialgébrico.

Corolário 1.11. A imagem de um conjunto semialgébrico por uma aplicação polinomial é um conjunto semialgébrico.

\subsection{Estratificações e triangulações}

A teoria de estratificação é fundamental na construção da trivialização topológica de família de variedades on família de aplicações. $\Lambda$ noção chave na teoria de estratificação são as condições de regularidade entre os estratos. Embora muitas condições de regularidade sejam conhecidas, citamos no texto apenas as condições de regularidade de Whitney e a (c)-regularidade de Bekka. Podemos encontrar mais informações sobre regularidade no artigo de Trotman |64|. L'm tipo de estratificação que será usadá neste trabalho, no Capítulo 6, é uma estratificação semialgébrica finita dada por A. Parusinski [47]. Esta estratificação dá a constância do tipo Lipschitz ao longo de cada estrato, o qual é mais forte do que a equisingularidade dada pela estratificação do Whitney.

Definição 1.12. Uma $C^{k}$-estratificação de um conjunto $X \subset \mathbb{R}^{n}$ é uma partição $X=\bigcup_{i-1}^{k} \mathcal{X}_{i}$ satisfazendo as condiçôes:

i) Cada $\mathcal{X}_{i}$ é subvariedade diferenciável de classe $C^{k}$, a qual chamamos de estrato.

ii) Os estratos são disjuntos, isto é, $\mathcal{X}_{i} \cap \mathcal{X}_{j}=\emptyset$, para todo $i, j$ com $i \neq j$.

iii) (Condição de Fronteira) Para todo par de estratos $\mathcal{X}_{i}, \mathcal{X}_{j}$, se $\mathcal{X}_{i} \cap \overline{\mathcal{X}_{j}} \neq 0$ entãa $\mathcal{X}_{i} \subset \overline{\mathcal{X}_{j}}$.

Teorema 1.13. (Lojasiewicz [29]) Todo conjunto semialgébrico admite uma $C^{k}$-estratificação.

Exemplo 1.14. Seja $X=\left\{(x, y) \in \mathbb{R}^{2} \mid x y=0\right\}$. O conjunto $X$ admite a seguinte estratificas:ão: $\mathcal{X}_{1}=\{(x, y) \mid x>0\}, \mathcal{X}_{2}=\{(x, y) \mid x<0\}, \mathcal{X}_{3}=\{(x, y) \mid y>0\}, \mathcal{X}_{4}=$ $\{(x, y) \mid y<0\}$ e $\mathcal{X}_{5}=\{(0,0)\}$ 


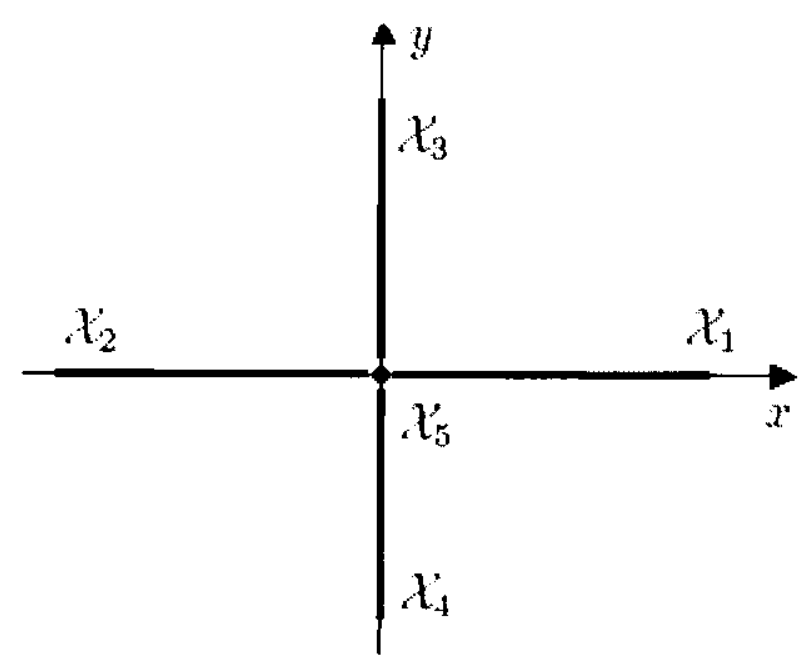

Figura 1.1: Estratificação dos eixos cartesianos

Exemplo 1.15. O guarda-chuva de Whitney $X=\left\{(x, y, z) \in \mathbb{R}^{3} \mid x^{2}-z y^{2}=0\right\}$ admite, por exemplo, uma estratificaşäo dada por um estrato de dimensãa 1 (o eixo-z) e dois outros estratos de dimensão 2 que forman o complementar do eixo-z $\mathrm{cm} X$.

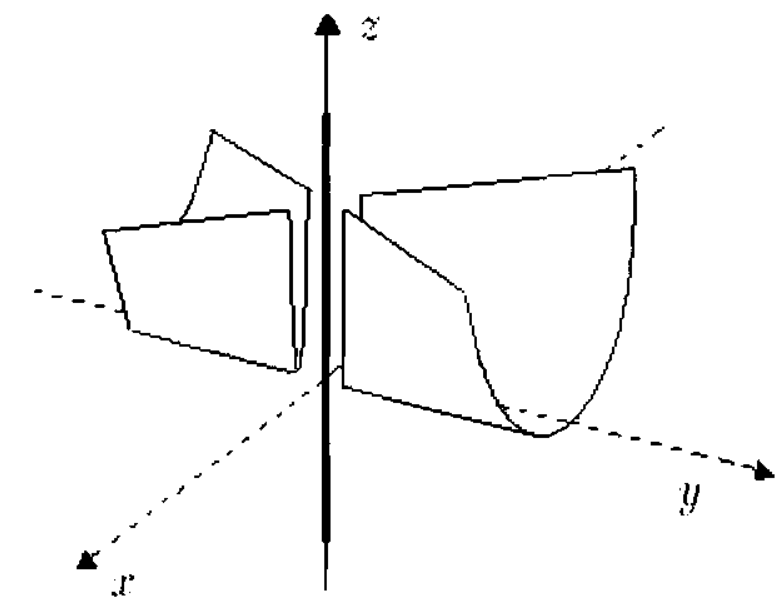

Figura 1.2: Estratificação do guarda-chuva de Whitney

O Exemplo 1.15 mostra que o tipo topológico dos pontos ao longo de um mesmo estrato pode não ser constante. Para resolver este tipo de problema, Whitney cria as condições de regularidade entre os estratos.

Sejann $\mathcal{X}$ e $\mathcal{Y}$ subvariedades do $\mathbb{R}^{n}$ de dimensões $r$ e $s$, respectivamente e $s \leq r$. Dizemos que o par $(\mathcal{X}, \mathcal{Y})$ satisfaz a condiça ${ }^{\prime}(a)$ de Whitney em um ponto $y \in \mathcal{Y}$ se para qualquer sequência de pontos $\left(x_{i}\right) \subset \mathcal{X}$ convergindo para $y$ tal que os espaços tangentes a $\mathcal{X}$ em $x_{i}, T_{x_{i}} \mathcal{X}$, convergem para algum $r$-plano $\tau\left(\subset \mathbb{R}^{n}\right)$, temos que $T_{y} \mathcal{Y} \subset \tau$. 
Observe que no Exemplo 1.15, o estrato de dimensão 2 não satisfaz a condição de (a)-regularidade sobre o eixo- $z$ na origem.

Dizemos que o par $(\mathcal{X}, \mathcal{Y})$ satisfaz a condição $(b)$ de: Whitney em um ponto $y \in \mathcal{Y}$ se dadas as sequências $\left(x_{i}\right) \subset \mathcal{X}$ convergindo para $y$ e $\left(y_{i}\right) \subset \mathcal{Y}$ convergindo para $y\left(x_{i} \neq y_{i}\right)$ de forma que os espaços tangentes $T_{x_{i}} \mathcal{X}$ convergem para algum r-plano $\tau\left(\subset \mathbb{R}^{n}\right)$ e as secantes $\widehat{x_{i} y_{i}}$ ligando $x_{i}$ a $y_{i}$ convergem para alguma reta $l\left(\subset \mathbb{R}^{n}\right)$, temos que $l \subset \tau$.

A condição (b) implica na condição $(a)$. Assim, no Exemplo 1.15 também não temos a (b)-regularidade na origem para o par de estratos (complementar do eixo- $z$ em $X$, eixo- $z$ ).

Dizemos que $(\mathcal{X}, \mathcal{Y})$ satisfaz a condição $(a)$ (resp. $(b))$ se satisfaz a condição $(a)$ (resp. (b)) para todo ponto $y \in \mathcal{Y}$.

Definição 1.16. Uma estratificação $\Xi$ de um conjunto $X \subset \mathbb{R}^{n}$ é Whitney regular se as condições $(a)$ e (b) são satisfeitas para todo par de estratos $(\mathcal{X}, \mathcal{Y})$ de $\Xi$.

Exemplo 1.17. Seja $X=\left\{(x, y, z) \in \mathbb{R}^{3} \mid x^{2}-z y^{2}=0\right\}$. Considere a seguinte estratificação de $X: \mathcal{X}_{1}=\{(0,0,0)\}, \mathcal{X}_{2}=\{(0,0, z) \mid z>0\}, \mathcal{X}_{3}=\{(0,0, z) \mid z<0\}$ e dois outros estratos de dimensão $2, \mathcal{X}_{4}$ e $\mathcal{X}_{\overline{5}}$, que formam o complementar do eixo- $z$ em $X$. Então, com esta estratificação, $X$ é Whitney regular.

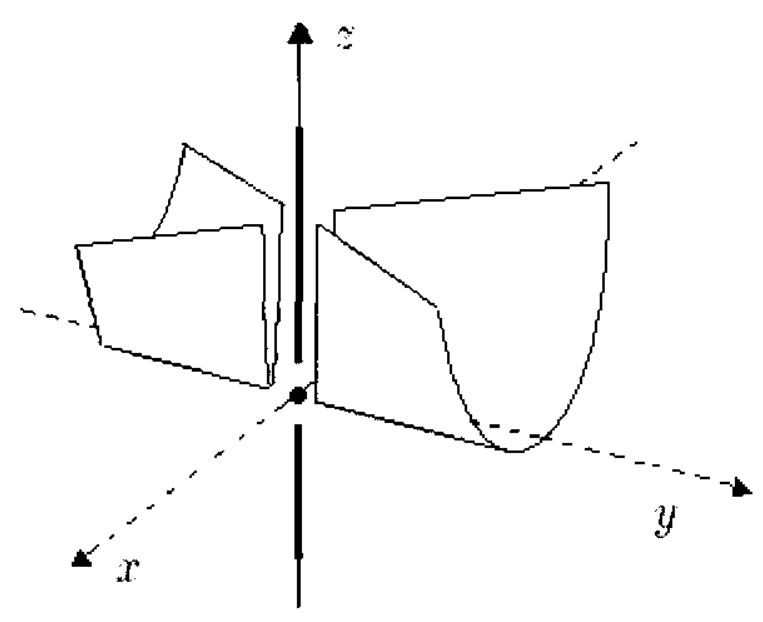

Figura 1.3: Estratificação Whitney regular do guarda-chuva de Whitney

Teorema 1.18. ([24]) Todo conjunto semialgébrico $X \subset \mathbb{R}^{n}$ admite uma estratificação de Whitney tendo um número finito de estratos semialgébricos.

Sejam $\Xi_{1}$ e $\Xi_{2}$ estratificaçốs de Whitney dos conjuntos $X_{1} \subset \mathbb{R}^{n}$ e $X_{2} \subset \mathbb{R}^{m}$, respectivamente. Uma aplicação contínua $f: X_{1} \rightarrow X_{2}$ é uma aplicação estratificada se as seguintes condições são satisfeitas: 
i) $f$ aplica estrato em estrato;

ii) Se $\mathcal{X}$ é um estrato de $\Xi_{1}$ aplicado por $f$ num estrato $\mathcal{X}^{\prime}$ de $\Xi_{2}$, então $f_{\left.\right|_{\mathcal{X}^{\prime}}}: \mathcal{X} \rightarrow \mathcal{X}^{\prime}$ é uma submersão.

Sejam $\mathcal{X}$ e $\mathcal{Y}$ subvariedades do $\mathbb{R}^{n}$ e seja $f: U \rightarrow \mathbb{R}^{m}$ uma aplicação suave definida em uma vizinhança $U$ de $\mathcal{X} \cup \mathcal{Y}$ em $\mathbb{R}^{n}$. Suponha que as restriçôes

$$
f_{\left.\right|_{\mathcal{X}}}: \mathcal{X} \rightarrow \mathbb{R}^{m} \text { e } f_{\mid \mathcal{Y}}: \mathcal{Y} \rightarrow \mathbb{R}^{m}
$$

tenham posto constante. Então, dizemos que $\mathcal{X}$ é I'hom regular sobre $\mathcal{Y}$ relativo a $f$ (ou o $\operatorname{par}(\mathcal{X}, \mathcal{Y})$ satisfaz a condiçäo $\left.a_{f}\right)$ se para qualquer sequência $\left(x_{i}\right) \subset \mathcal{X}$ convergindo para $y$ tal que a sequência dos planos ker $\mathrm{d}\left(f_{!_{\mathcal{X}}}\right)_{x_{i}}$ converge para um plano $k$, temos que ker $\mathrm{d}\left(f_{\left.\right|_{y}}\right)_{y} \subset k$, onde ker $\mathrm{d}\left(f_{\left.\right|_{\mathcal{X}}}\right)_{x}$ denota o kernel da diferencial $\mathrm{d}\left(f_{\left.\right|_{\mathcal{X}}}\right)_{x}: T_{\mathrm{x}} \mathcal{X} \rightarrow T_{f(x)} \mathbb{R}^{m}$ de $f_{\mid \mathcal{X}}$ em $x$.

Sejam $X_{1} \subset \mathbb{R}^{n}, X_{2} \subset \mathbb{R}^{m}$ e $\Xi_{1}, \Xi_{2}$ estratificações de Whitney de $X_{1}$ e $X_{2}$, respectivamente. Dizemos que $f: X_{1} \rightarrow X_{2}$ é uma aplicaçấo de Thom se $f$ ć estratificada c se para qualquer par de estratos de $\Xi_{1}$ a condição de regularidade de Thom (condição $a_{f}$ ) é satisfeita.

Teorema 1.19. (Segundo Lema de Isotopia de Thom, [36]) Sejam $f: X_{1} \rightarrow X_{2}$ uma aplicação própria de Thom e $g: X_{2} \rightarrow V$ uma aplicação própria estratificada, com respeito às estratificações $\Xi_{1}, \Xi_{2} e\{V\}, V$ uma variedade conexa. Então, para quaisquer dois pontos $p, q \in V$, as aplicações restritas $f_{i_{E_{p}}}: E_{p} \rightarrow F_{p}$ e $f_{\left.\right|_{r_{q}}}: E_{q} \rightarrow F_{q}$ são topologicamente equivalentes, onde $E_{p}=(g \circ f)^{-1}(p)$ e $F_{p}=g^{-1}(p)$. Isto é, existem homeomorfismos $h_{1}: E_{p} \rightarrow E_{q}$ e $h_{2}: F_{p} \rightarrow F_{q}$ tais que $\left(f_{\left.\right|_{E_{q}}}\right) \circ h_{1}=h_{2} \circ\left(f_{\left.\right|_{E_{p}}}\right)$.

Além das condições (a) e (b) de Whitney, destacamıs a (c)-regularidade introduzida por K. Bekka (|3|). A condição (c) será usada em nossos resultados do Capítulo 5.

Definição 1.20. Sejam $\mathcal{X}, \mathcal{Y}$ subvariedades do $\mathbb{R}^{n} \operatorname{com} \mathcal{Y} \subset \overline{\mathcal{X}}$ e $\rho: \mathbb{R}^{n} \rightarrow \mathbb{R}$ uma função não-negativa tal que $\rho^{-1}(0)=\mathcal{Y}$. $O$ par $(\mathcal{X}, \mathcal{Y})$ é $(\mathrm{c})$-regular em $y_{0} \in \mathcal{Y}$ com respeito a função $\rho$ se, dada uma sequêneia $\left(x_{i}\right) \subset \mathcal{X}$ convergindo para yo tal que a sequência dos planos $\left\{\operatorname{ker}\left(\mathrm{d} \rho\left(x_{i}\right)\right) \cap T_{x_{i}} \mathcal{X}\right\}$ converge para um plano $\tau$, então $T_{y_{0}} \mathcal{Y} \subset \tau$. ( par $(\mathcal{X}, \mathcal{Y})$ é (c)-regular com respeito a função $\rho$ se ele é (c)-regular para qualquer $y_{0} \in \mathcal{Y}$ com respeito a função $\rho$.

A condição de (c)-regularidade implica na condição (a) de Whitncy. 
Scjam $\left(T_{\mathcal{Y}}, \pi, \rho\right)$ uma vizinhança tubular de $\mathcal{Y}$ junto com uma projeção $\pi: T_{\mathcal{Y}} \rightarrow \mathcal{Y}$, associada a uma função não-negativa $\rho$ tal que $\rho^{-1}(0)=\mathcal{Y}$ e $\operatorname{grad} \rho(x) \in \operatorname{ker}(\mathrm{d} \pi(x))$, onde grad representa o gradiente.

Definição 1.21. O par $(\mathcal{X}, \mathcal{Y})$ satisfaz a condição $(\mathrm{m})$ se existe um nímero real positivo $\varepsilon>0$ tal que

$$
\begin{aligned}
(\pi, \rho)_{\mid \mathcal{X} \cap T_{\mathcal{Y}}^{\beta}}: \mathcal{X} \cap T_{\mathcal{Y}}^{\mathcal{\varepsilon}} & \rightarrow \mathcal{Y} \times[0, \varepsilon) \\
x & \mapsto(\pi(x), \rho(x))
\end{aligned}
$$

é uma submersĩo, onde $T_{\mathcal{Y}}^{\varepsilon}:-\left\{x \in T_{\mathcal{Y}} \mid \rho(x)<\varepsilon\right\}$.

A seguinte caracterização da (c)-regularidade é também devida à Bckka (ver [4]).

Proposição 1.22. O par $(\mathcal{X}, \mathcal{Y})$ é (c)-regular em $y_{0} \in \mathcal{Y}$ com respeito a função $\rho$ se, $e$ somente se, o par $(\mathcal{X}, \mathcal{Y})$ é $(a)$-regular em $y_{0} \in \mathcal{Y}$ e satisfaz a condição $(\mathrm{m})$.

Uma estratificação $\Xi$ é (c)-regular com respeito a função $\rho$ se para qualquer par de estratos $(\mathcal{X}, \mathcal{Y})$ de $\Xi$, temos que $(\mathcal{X}, \mathcal{Y})$ é $(c)$-regular com respeito a função $\rho$.

Na segunda parte desta Seção 1.2, introduzimos o conceito de triangulação. Lembramos que cm Topologia Geral ou Algébrica, conjuntos que admitem estratificaçõos o triangulações são interessantes, pois apresentam boas propriedades métricas e geométricas.

Definição 1.23. Seja $e_{1}, \ldots, e_{n+1}$ a base canônica de $\mathbb{R}^{n+1}$. Denominaremos de nsimplexo padrão o conjunto $\Delta_{n}=\left\{\sum_{i=1}^{n+1} \lambda_{i} \epsilon_{i} \mid \sum_{i=1}^{n+1} \lambda_{i}=1,0 \leq \lambda_{i} \leq 1\right\}$. A fronteira de um n-simplexo padrão é dada por,

$$
\partial \Delta_{n}=\left\{\sum_{i=1}^{n+1} \lambda_{i} e_{i} \mid \sum_{i=1}^{n+1} \lambda_{i}=1, \text { e } \exists i \operatorname{com} \lambda_{i}=0\right\} .
$$

Definiçāo 1.24. Um conjunto $X \subset \mathbb{R}^{n+1}$ é um complexo simplicial padrão se existe um $n$-simplexo padrão $\Delta_{n}$, tal que $X=\bigcup_{i=1}^{k} X_{i}$ e, para cada $i$, existe um conjunto de indices $I_{i} \subset\{1, \ldots, n+1\}$, de sorte que

$$
X_{i}=\left\{\sum_{j \in I_{i}} \lambda_{j} e_{j} \mid \sum_{j \in I_{i}} \lambda_{i}=1,0 \leq \lambda_{j} \leq 1\right\} \subset \underbrace{\partial \ldots \partial}_{p_{i} \text { vezes }} \Delta_{n}
$$

para algum $p_{i}$ inteiro positivo.

Definiçāo 1.25. Um conjunto $X \subset \mathbb{R}^{n}$ é triangulável se existe um complexo simplicial padrão $Y \subset \mathbb{R}^{k}$ e uma aplicação $h: X \rightarrow Y$, tal que h é um homeomorfismo. $A$ aplicação $h$ é chamada de triangulação de $X$. A triangulação $h$ é dita semialgébrica se $h$ é uma aplicação semialgébrica (isto é, graf $(h)$ é um conjunto semialgébrico). 
Teorema 1.26. (Teorema de 'Triangulação de Lojasieuicz (29)) Todo conjunto semialgébrico compacto admite uma triangulação semialgébrica.

Teorema 1.27. (Lema de Seleşão da Curva [37)) Sejam $U \subset \mathbb{R}^{m}$ um conjunto algébrico e $V \subset \mathbb{R}^{n}$ um conjunto semialgébrico. Se $U \cap V$ contém pontos arbitrariamente próximos da origem (isto é, $0 \in \overline{U \cap V}$ ) então, existe uma curva analitica real $\gamma:[0, \varepsilon) \rightarrow \mathbb{R}^{m}$ tal que $\gamma(0)=0$ e $\gamma(t) \in U \cap V$, para todo $t>0$.

\subsection{Conjuntos analíticos, semianalíticos e subanalíticos}

As informações que introduzimos para conjuntos semialgébricos podem ser estabelecidas para outros conjuntos, num sentido bem mais geral, como é o caso dos conjuntos semianalíticos e subanalíticos.

Definição 1.28. Um conjunto $X \subset \mathbb{R}^{n}$ é analítico se para todo $x \in X$, existe uma vizinhança $U$ de $x$ em $\mathbb{R}^{n}$ e urna função analítica $f: U \rightarrow \mathbb{R}$, tal que $X \cap U=f^{-1}(0)$.

Todo conjunto algébrico é analítico, porêm nem todo conjunto analítico ê algébrico. Por exemplo, $f^{-1}(0)$ onde $f(x, y)=y-\ln (x)$.

Definição 1.29. Um conjunto $X \subset \mathbb{R}^{n}$ é scmianalítico básico se para todo $x \in X$, existe uma vizinhança $U$ de $x$ em $\mathbb{R}^{n}$ e funçöes analiticas $f, g_{1}, \ldots, g_{k}: U \rightarrow \mathbb{R}$, tais que

$$
X \cap U=\left\{x \in \mathbb{R}^{n} \mid f(x)=0\right\} \cap\left(\bigcap_{i=1}^{k}\left\{x \in \mathbb{R}^{n} \mid g_{i}(x)>0\right\}\right) .
$$

Definição 1.30. Um conjunto semianalítico é uma reunião finita de conjuntos semianalíticos básicos.

A semelhança que cxiste cntre a definição de conjunto semianalítico e a definição dada na Seção 1.1 para conjunto semialgébrico, justifica o fato de que muitos resultados válidos para os conjuntos semialgébricos também valham para os conjuntos semianalíticos, mesmo estes últimos tendo uma definição de caráter local. Porém, nem todos resultados são válidos como, por exemplo, o Teorema 1.10 de Tarski-Seidenberg. Isto nos motiva a definição de uma categoria muito mais geral que semianalíticos, a saber, os conjuntos subanalíticos.

Definição 1.31. Um conjunto $X \subset \mathbb{R}^{n}$ é subanalítico se existe um conjunto $\tilde{X} \subset \mathbb{R}^{m}$, $m \geq n$, semianalitico, tal que a projeção $\pi: \mathbb{R}^{m} \rightarrow \mathbb{R}^{n}$ restrita a $\tilde{X}$ é uma aplicação própria $e X=\pi(\tilde{X})$. 
Com esta definição o Teorema de Tarski-Seidenberg torna-se verdadeiro.

Podemos citar como propriedades dos conjuntos subanalíticos:

a) Todo conjunto semianalítico é subanalítico.

b) Se $X, Y$ são conjuntos subanalíticos então $X \cup Y, \mathbb{R}^{n}-X$ (Teorema de Gabrielov $[20 \mid), \mathbb{R}^{n}-Y$ (Teorema de Gabrielov [20]), $X \cap Y$ e $X-Y$ são também subanalíticos.

c) Se $X \subset \mathbb{R}$ ć um conjunto subanalítico, então $X$ é reunião finita de intervalos abertos, fechados, semiabertos e pontos.

\subsection{Estruturas o-minimais}

O estudo das estruturas o-minimais iniciou-se por volta de 1994, com Khovanski e van den Dries, entre outros. A principal característica das estruturas o-minimais ć que não ocorrem "fenômenos ruins" nestas estruturas. Por exemplo, considere o caso patológico do gráfico da função real $f(x)=\operatorname{sen}\left(\frac{1}{x}\right)$, para $x>0$. É conhecido que o fecho do gráfico de $f$ é conexo mas não é conexo por caminhos. As estruturas o-minimais admitem "dlesenvolver uma topologia bem comportada" de modo que tais fenômenos ruins não aconteçam. $O$ modelo para as estruturas o-minimais é a classe dos conjuntos scmialgébricos. Como já vimos, a classe destes conjuntos é "estável" por muitas construções, como por cxemplo, projeções, fecho, componentes conexas, ctc. c, por outro lado, a topologia dos conjuntos semialgébricos é muito simples, sem patologias. Assim, as estruturas o-minimais podem ser vistas como um tratamento axiomático da geometria semialgébrica. Para maiores detalhes sobre estruturas o-minimais ver [14].

Definiçāo 1.32. Uma estrutura expandindo um corpo real fechado $R$ (ou por simplicidade, $\mathbb{R})$ é uma coleção $\mathcal{S}=\left(\mathcal{S}_{n}\right)_{n \in \mathbb{N}}$, onde cada $\mathcal{S}_{n}$ é um conjunto de subconjuntos do espaço afim $R^{n}$, satisfazendo os seguintes axiomas:

i) Todos subconjuntos algébricos de $R^{n}$ estão em $\mathcal{S}_{n}$.

ii) Para todo $n, \mathcal{S}_{n}$ é uma álgebra finita, isto é, se $A, B \in \mathcal{S}_{n}$ então $A \cap B, A \cup B, A^{c}$ (complementar de $A$ ), $B^{c}$ (complementar de $B$ ) pertencem a $\mathcal{S}_{n}$

iii) Se $A \in \mathcal{S}_{n}$ e $B \in \mathcal{S}_{m}$ então $A \times B \in \mathcal{S}_{n+m}$.

iv) Se $\pi: R^{n+1} \rightarrow R^{n}$ é a projeção nas $n$ primeiras coordenadas e $A \in \mathcal{S}_{n+1}$ então $\pi(A) \in \mathcal{S}_{n}$ (propriedade de Tarski) 
Os elementos de $\mathcal{S}_{n}$ são chamados de subconjuntos definíveis de $R^{n}$. A estrutura $\mathcal{S}$ chamada o-minimal sé, além das condições acima satisfaz também:

v) Os elementos de $\mathcal{S}_{1}$ são precisamente as uniões finitas de pontos e intervalos.

Uma função $f$ é chamada definível em $\mathcal{S}$ se o $\operatorname{graf}(f)$ é um conjunto definível em $\mathcal{S}$.

Exemplo 1.33. Todo subconjunto semialgébrico de $\mathbb{R}^{n}$ é definivel e o conjunto de todos os conjuntos semialgébricos é uma estrutura o-minimal.

O teorema a seguir apresenta boas consequências topológicas que aparecom a partir de um conjunto definível.

Teorema 1.34. (van den Dries [15]) Seja X um conjunto definivel em uma estrutura o-minimal $\mathcal{S}$. Então,

i) X é triangulável e o homeomorfismo da triangulação é definivel (isto é, o seu gráfico é um conjunto definivel).

ii) Para todo k, $X$ admite uma $C^{k}$-estratificação.

Teorema 1.35. (Lema de Seleção da Curvva (14l) Seja A um subconjunto definível em $R^{n}$ $e b \in \bar{A}$. Então, existe uma aplicação continua definível $\gamma:[0,1) \rightarrow R^{n}$ tal que $\gamma(0)=b$ $e \gamma((0,1)) \subset A$

\subsection{Resultados da teoria de singularidades}

Antes de iniciarmos a segunda parte do Capítulo 1, que trata dos resultados clássicos da teoria de singularidades, vamos definir algumas notações e enunciar um teorema de transversalidade para varicdades diferenciáveis.

Dado $\varepsilon>0$, definimos

$D_{\varepsilon}^{n}=\left\{\left(x_{1}, \ldots, x_{n}\right) \in\left(\mathbb{R}^{n}, 0\right) \mid x_{1}^{2}+\ldots+x_{n}^{2} \leq \varepsilon\right\}$,

$B_{\varepsilon}^{n}=\left\{\left(x_{1}, \ldots, x_{n}\right) \in\left(\mathbb{R}^{n}, 0\right) \mid x_{1}^{2}+\ldots+x_{n}^{2}<\varepsilon\right\}$,

$S_{\varepsilon}^{n-1}=\left\{\left(x_{1}, \ldots, x_{n}\right) \in\left(\mathbb{R}^{n}, 0\right) \mid x_{1}^{2}+\ldots+x_{n}^{2}=\varepsilon\right\}$. A esfera padrão unitária, conforme já citada antes, será indicada apenas por $S^{n-1}$.

Teorema 1.36. (Lema de Transversalidade de Thom [23]) Seja $f: \mathbb{R}^{n} \times \mathbb{R}^{s} \rightarrow \mathbb{R}^{p}$ uma familia de aplicacöes transversais às variedades $\mathcal{X}_{1}, \ldots, \mathcal{X}_{t}$ de $\mathbb{R}^{p}$. Então, existe um conjunto denso de parâmetros s para os quais $f_{s}: \mathbb{R}^{n} \rightarrow \mathbb{R}^{p}, f_{s}(x)=f(x, s)$, é transversal a $\mathcal{X}_{1}, \ldots, \mathcal{X}_{t}$ 
Os aspectos básicos da teoria de singularidades dos germes de aplicações diferenciáveis estão, atualmente, bem difundidos. As definições e resultados desta seção estão, em sua maioria $\mathrm{em}[23]$ e [65].

Nosso objetivo é o estudo de propriedades locais de aplicações diferenciáveis de classe $\mathcal{C}^{\infty}, \quad f: \mathbb{R}^{n} \rightarrow \mathbb{R}^{p}$. Por esta razão, trabalhamos com germes de aplicação em um ponto $a \in \mathbb{R}^{n}$, isto é, classes de equivalências de aplicações que coincidem em alguma vizinhança de $a$. Os pontos $a \in \mathbb{R}^{n}$ e $f(a) \in \mathbb{R}^{p}$ são chamados, respectivamente, fonte e meta do germe. Notação: $f:\left(\mathbb{R}^{n}, a\right) \rightarrow\left(\mathbb{R}^{p}, f(a)\right)$. Sem perda de generalidade, vamos assumir $a=0$.

Utilizando convenientes sistemas de coordenadas, consideraremos simplesmente

$$
f=\left(f_{1}, \ldots, f_{p}\right):\left(\mathbb{R}^{n}, 0\right) \longrightarrow\left(\mathbb{R}^{p}, f(0)\right),
$$

como sendo um germe de aplicação diferenciável de classe $C^{\infty}$. Para germes que aparecem no decorrer do texto e não são de classe $C^{\infty}$, faremos menção explícita da sua classse de diferenciabilidade.

O conjunto de todos os germes de aplicação $C^{\infty}, f:\left(\mathbb{R}^{n}, 0\right) \longrightarrow\left(\mathbb{R}^{p}, f(0)\right)$ scrá denotado por $\mathcal{E}_{n, p}$. Quando $p=1$, indicaremos apenas por $\mathcal{E}_{n}$. O anel $\mathcal{E}_{n}$ é local e tem como único ideal maximal $m_{n}=\left\{f:\left(\mathbb{R}^{n}, 0\right) \rightarrow \mathbb{R} \mid f(0)=0\right\}=\left\langle x_{1}, \ldots, x_{n}\right\rangle$. A classe de equivalência das aplicações cujas derivadas na origem coincidem até a ordem $r$ no desenvolvimento de Taylor omitindo-se o termo constante, é denominada $r$-jato de $f$ na origem, e a notação usada é $j^{r} f(0)$. O conjunto de todos os $r$-jatos ć denotado por $J^{r}(n, p)$. Um resultado elementar nos mostra que $j^{r} f(0)=0 \Leftrightarrow f \in m_{n}^{r+1}$.

Denotaremos a $\mathbb{R}$-álgebra local de $f$ por $Q(f)=\mathcal{E}_{n} /\langle f\rangle$, onde $\langle f\rangle=\left\langle f_{1}, \ldots, f_{p}\right\rangle$ é o ideal de $\mathcal{E}_{n}$ gerado pelas funções coordenadas de $f$. O conjunto singular de $f$, formado pelos pontos não submersivos de $f$, será denotado por $\Sigma f$.

Seja $T \mathbb{R}^{n}$ o fibrado tangente a $\mathbb{R}^{n}$.

Um campo de vetorcs ao longo de $f:\left(\mathbb{R}^{n}, 0\right) \rightarrow\left(\mathbb{R}^{p}, 0\right)$ é um germe $\xi:\left(\mathbb{R}^{n}, 0\right) \rightarrow \mathrm{T} \mathbb{R}^{p}$ tal que $\pi_{p} \circ \xi=f$, como no diagrama a seguir.

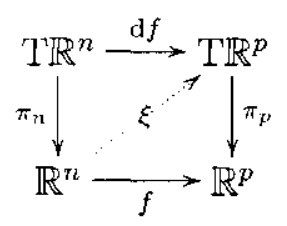

Seja $\theta_{f}$ o conjunto de todos os campos de vetores ao longo de $f$. Em particular, quando consideramos $f$ como a aplicação identidade do $\mathbb{R}^{n}$, indicaremos o conjunto dos campos ao longo da identidade por $\theta_{n}$. 
Considere a aplicação

$$
\begin{aligned}
t f: \theta_{n} & \longrightarrow \theta_{f} \\
\phi & \mapsto \mathrm{d} f \circ \phi,
\end{aligned}
$$

onde $\mathrm{d} f$ é a diferencial de $f$.

Com a identificação de $T \mathbb{R}^{n}$ com $\mathbb{R}^{n} \times \mathbb{R}^{n}$, podemos identilicicar $\theta_{f}$ com $\mathcal{E}_{n, p}$. Assim, $\theta_{f}$ é um $\mathcal{E}_{n}$-módulo livre de posto $p$, e $\theta_{n}$ é um $\mathcal{E}_{n}$-módulo livre de posto $n$. Com isso, a aplicação tf é um homomorfismo entre $\mathcal{E}_{n}$-módulos livres.

Vários grupos agem em $\mathcal{E}_{n, p}$. Quando dois clementos $f, g \in \mathcal{E}_{n, p}$ estão cm uma mesma órbita segundo a ação de um grupo $G$, dizemos que $f$ e $g$ são $G$-equivalentes e denotamos por $f \stackrel{G}{\sim} g$.

As relações de equivalência de nosso interesse são aquelas definidas pelas ações dos seguintes grupos (de Mather):

Definição 1.37. Seja $\int:\left(\mathbb{R}^{n}, 0\right) \rightarrow\left(\mathbb{R}^{p}, 0\right)$. Definimos:

$\mathcal{R}=$ grupo dos yermes de difeomorfismos $h:\left(\mathbb{R}^{n}, 0\right) \rightarrow\left(\mathbb{R}^{n}, 0\right)$ cuja açäo em $f$ é a composição à direita, isto é, $f \circ h^{-1}$.

$\mathcal{L}=$ grupo dos germes de difeomorfismos $k:\left(\mathbb{R}^{p}, 0\right) \rightarrow\left(\mathbb{R}^{p}, 0\right)$ cuja ação em $f$ é a composição à esquerda, isto é, $k \circ f$.

$\mathcal{A}=\mathcal{R} \times \mathcal{L}$ grupo das mudanşas de coordenadas na fonte c na meta. $A$ ação em $f$ é dada por $k \circ \int \circ h^{-1}$.

$\mathcal{C}=$ grupo dos germes de difeomorfismos $H:\left(\mathbb{R}^{n} \times \mathbb{R}^{p}, 0\right) \rightarrow\left(\mathbb{R}^{n} \times \mathbb{R}^{p}, 0\right)$ tais que $H(x, y)=(x, 0(x, y))$ e $H(x, 0)=(x, 0)$. Se $\theta_{x}$ é um difeomorfismo de $\mathbb{R}^{p}$, dependendo de $x \in \mathbb{R}^{n}$, sua ação tranısforma $f$ na aplicação

$$
x \mapsto \theta_{x}(f(x)) .
$$

Equivalentemente, $H$ age no gráfico de $f$ em $\mathbb{R}^{n} \times \mathbb{R}^{p}$.

$\mathcal{K}=\mathcal{R} \cdot \mathcal{C}$ (produto semi-direto). É o grupo formado pelos germes de difeomorfismos $H:\left(\mathbb{R}^{n} \times \mathbb{R}^{p}, 0\right) \rightarrow\left(\mathbb{R}^{n} \times \mathbb{R}^{p}, 0\right)$ que süo escritos na forma $H(x, y)=(h(x), \theta(x, y))$, com $h \in \mathcal{R}$ e $H(x, 0)=(h(x), 0)$. O diagrama comutativo abaixo descreve uma $\mathcal{K}$-equivalência entre os germes $f$ e $g:\left(\mathbb{R}^{n}, 0\right) \rightarrow\left(\mathbb{R}^{p}, 0\right)$ :

$$
\begin{array}{ccccc}
\left(\mathbb{R}^{n}, 0\right) & \stackrel{(i d, f)}{\longrightarrow} & \left(\mathbb{R}^{n} \times \mathbb{R}^{p}, 0\right) & \stackrel{\pi_{n}}{\longrightarrow} & \left(\mathbb{R}^{n}, 0\right) \\
h \downarrow & H \downarrow & & h \downarrow \\
\left(\mathbb{R}^{n}, 0\right) & \stackrel{(i d, g)}{\longrightarrow} & \left(\mathbb{R}^{n} \times \mathbb{R}^{p}, 0\right) & \stackrel{\pi_{n}}{\longrightarrow} & \left(\mathbb{R}^{n}, 0\right)
\end{array}
$$


onde id $:\left(\mathbb{R}^{n}, 0\right) \rightarrow\left(\mathbb{R}^{n}, 0\right)$ é a aplicação identidade do $\mathbb{R}^{n}$ e $\pi_{n}:\left(\mathbb{R}^{n} \times \mathbb{R}^{p}, 0\right) \rightarrow\left(\mathbb{R}^{n}, 0\right)$ a projeção canônica. Note que $H$ age no gráfico da $f$, aplicando-o no gráfico da $g$.

Notação. $f \stackrel{\mathcal{K}}{\sim} g$.

O grupo $\mathcal{K}$ é chamado grupo de contato. O grupo $\mathcal{C}$ é subgrupo normal de $\mathcal{K}$ e os grupos $\mathcal{R}, \mathcal{L}$ e $\mathcal{A}$ podem ser identificados com subgrupos de $\mathcal{K}$.

Embora os grupos de Mather não sejam grupos de Lie e $\mathcal{E}_{n, p}$ não seja variedade, é possível seguir o modelo finito de grupos de Lic agindo em variedades e definir objetos que são chamados espaços tangentes à órbita de um germe $f$, com respeito a um destes grupos. Como temos especial interesse pelo grupo de contato $\mathcal{K}$, definiremos espaço tangente $\mathrm{c}$ codimensão apcnas para $\mathcal{K}$. Para os outros grupos podemos ver [23], por exemplo.

Definição 1.38. Seja $f:\left(\mathbb{R}^{n}, 0\right) \rightarrow\left(\mathbb{R}^{p}, 0\right)$. O espaço tangente ao grupo $\mathcal{K} \mathrm{em} f$ é definido por

$$
T_{f} \mathcal{K}:=t f\left(m_{n} \theta_{n}\right)+f^{\star}\left(m_{p}\right) \theta_{f}
$$

onde $f^{\star}\left(m_{p}\right) \theta_{f}$ é o módulo formado por $p$ cópias do ideal gerudo pelas funçôes coordenadas de $f=\left(f_{1}, \ldots, f_{p}\right)$. Quando não fixamos a origem, definimos o espaço tangente estendido de $\mathcal{K}$ em f por

$$
T_{f} \mathcal{K}_{e}:=t f\left(\theta_{n}\right)+f^{\star}\left(m_{p}\right) \theta_{f}
$$

$\Lambda \mathcal{K}$-codimensão de $f$ é definida como a dimensão (como $\mathbb{R}$-espaço vetorial) do quo-

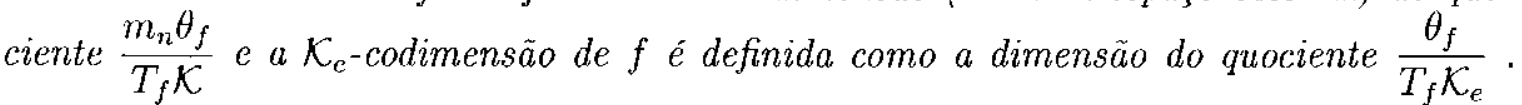

A classe dos germes que estudamos é a daqueles que podem scr representados pclo scu polinômio de Taylor de alguma ordem. A linguagem básica para isto envolve o conceito de determinação finita. Muitos trabalhos são dedicados à caracterização da determinação finita e outros buscam encontrar estimativas para a ordem de determinação, com respeito às várias relações de equivalência. Como estamos interessados particularmente no grupo $\mathcal{K}$, introduzimos o conceito de germes $\mathcal{K}$-finitamente determinados. Para os outros grupos de Mather a definição é análoga. A referência básica para estc assunto é [65]].

Definição 1.39. Um germe $f:\left(\mathbb{R}^{n}, 0\right) \longrightarrow\left(\mathbb{R}^{p}, 0\right)$ é $r-\mathcal{K}$-determinado se para todo germe $g:\left(\mathbb{R}^{n}, 0\right) \longrightarrow\left(\mathbb{R}^{p}, 0\right)$ com $j^{r} f(0)=j^{r} g(0)$ então $f$ é $\mathcal{K}$-equivalente a $g$.

Se $f$ é $r$ - K-determinado para algum $r<\infty$, então $f$ é $\mathcal{K}$-finitamente determinado ou $\mathcal{K}$-finito e r (o menor possivel) é o grau de determinação de $f$. 
A propricdade de $\mathcal{K}$-determinação finita é aberta, ou seja, se consideramos $f:\left(\mathbb{R}^{n+1}, 0\right) \rightarrow\left(\mathbb{R}^{p}, 0\right)$ com $f(0, t)=0$ e $f(x, 0)=f_{0}(x)$ um germe $\mathcal{K}$-finito, cntão $f_{t}:\left(\mathbb{R}^{n}, 0\right) \rightarrow\left(\mathbb{R}^{p}, 0\right)$ é $\mathcal{K}$-finito para todo $t$ suficientemente pequeno.

Os teoremas de determinação permitem que classificações de germes possam ser realizadas. A seguir reproduzimos alguns dos principais critérios e estimativas para a $\mathcal{K}$-determinação finita dos germes de aplicaçõos diferenciáveis.

Teorema 1.40. (Critério Infinitesimal para Determinação Finita [65]) São equivalentes:

a) $f$ é $\mathcal{K}$-finitamente determinado;

b) para algum $r, T_{f} \mathcal{K} \supseteq m_{n}^{r} \theta_{f}$

c) para algum $r, T_{f} \mathcal{K}+m_{n}^{r+1} \theta_{f} \supseteq m_{n}^{r} \theta_{f}$

d) a K-codimensão de f́ é finita;

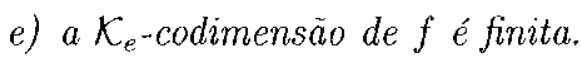

Mais precisamente,

i) sef ér-K-determinado então, $T_{f} \mathcal{K} \supseteq m_{n}^{r+1} \theta_{f}$

ii) se $T_{f} \mathcal{K} \supseteq m_{n}^{r+1} \theta_{f}$, então $f e ́(r+1)-\mathcal{K}$-determinado;

iii) se a $\mathcal{K}$-codimensão de $f$ éd, entäo $T_{f} \mathcal{K} \supseteq m_{n}^{d+1} \theta_{f}$.

A proposição a seguir relaciona a $\mathcal{K}$-determinação finita de um germe nas categorias: $C^{\infty}$ real, analítica real e analítica complexa (holomorfa).

Proposição 1.41. (Gaffney [21)) Se f é um germe de aplicação analítico real, então são equivalentes:

a) $f$ é $\mathcal{K}$-finilamente determinado como germe de aplicação $C^{\infty}$;

b) $f$ é $\mathcal{K}$-finitamenté determinado como germe analitico real;

c) a complexificação de $f$ é $\mathcal{K}$-finitamente determinada como germe de aplicação holomorfa.

As noções seguintes são de J.C. Tougeron [62|. Se $W_{r} \subset J^{r}(n, p)$ são conjuntos algébricos, com $W_{r+1} \subset \pi^{-1} W_{r}$, o conjunto $W$ dos germes de aplicações $f$ tais que $j^{r} f(0) \in W_{r}$, para todo $r$, é chamado de pró-algébrico. A codimensão de $W_{r}$ é menor ou igual a codimensão de $W_{r+1}$. A codimensão de $W$ é definida pelo limite das codimensões de $W_{r}$ 
quando $r \rightarrow \infty$. Dizemos que uma propriedade $\mathrm{P}$ dos germes de aplicações ocorre em geral se P ocorre exceto para um conjunto pró-algébrico de codimensão infinita.

Suponha $W$ pró-algébrico. Então, a codimensão de $W$ é infinita se, e somente se, para todo $z \in J^{r}(n, p)$, cxiste $f \notin W$ tal que $j^{r} f(0)=z$.

O teorema a seguir é um resultado devido a Mather e Tougeron (ver [62], [63]). O argumento de Mather aparece em [10]. O teorema pode também ser encontrado em [65] e [12].

Teorema 1.42. Germes de aplicação säo $\mathcal{K}$-finitamente determinados em geral.

$\Lambda$ demonstração deste tcorema usa o fato de que os germes que não são $\mathcal{K}$-finitos formam um conjunto pró-algébrico.

Os resultados a seguir apresentam caracterizações e invariantes conhecidos para a $\mathcal{K}$-equivalência

Teorema 1.43. (Mather [34]) Sejam $f, g:\left(\mathbb{R}^{n}, 0\right) \longrightarrow\left(\mathbb{R}^{p}, 0\right)$ germes $\mathcal{K}$-finitamente determinados. Então, $f$ é $\mathcal{K}$-equivalente a g se, e somente se, as $\mathbb{R}$-álgebras locais $Q(f)$ $e Q(g)$ são isomorfas.

Teorema 1.44. (Mather (34) Sejam $f, g:\left(\mathbb{R}^{n}, 0\right) \longrightarrow\left(\mathbb{R}^{p}, 0\right)$ germes estáveis. Então, $f$ é $\mathcal{A}$-equivalente a g se, e somente se, eles são $\mathcal{K}$-equivalentes.

A proposição seguinte apresenta uma caracterização algébrica da $\mathcal{K}$-equivalência de Mather.

Proposição 1.45. (Mather [3!]]) Sejam $f, g:\left(\mathbb{R}^{n}, 0\right) \longrightarrow\left(\mathbb{R}^{p}, 0\right)$. São equivalentes:

a) $f$ eg são $\mathcal{C}$-equivalentes;

b) os ideais das funçôes coordenadas de $f$ e g são iguais, isto é,

$$
\langle f\rangle=\left\langle f_{1}, \ldots, f_{p}\right\rangle=\left\langle g_{1}, \ldots, g_{p}\right\rangle=\langle g\rangle
$$

c) existe uma matriz inversivel $M, p \times p$, com coeficientes en $\mathcal{E}_{n}$, tal que $f=M \cdot g$.

Corolário 1.46. (Mather (39|) Sejam $f, g:\left(\mathbb{R}^{n}, 0\right) \longrightarrow\left(\mathbb{R}^{p}, 0\right)$. Säo equivalentes:

a) $f$ e g são $\mathcal{K}$-equivalentes;

b) existe um germe de difeomorfismo $h:\left(\mathbb{R}^{n}, 0\right) \longrightarrow\left(\mathbb{R}^{n}, 0\right)$ tal que $f$ e $g \circ h$ são $\mathcal{C}$-equivalentes; 
c) os ideais da.s funçótes coordenadas de $f$ e goh são iguais, isto ê,

$$
\langle f\rangle=\left\langle f_{1}, \ldots, f_{p}\right\rangle=\left\langle(g \circ h)_{1}, \ldots,(g \circ h)_{n}\right\rangle=\langle g \circ h\rangle ;
$$

d) existe uma matriz inversivel $M, p \times p$, com coeficientes em $\mathcal{E}_{n}$, tal que $f=M \cdot g \circ h$.

Observação. (O) conjunto dos pares $(M, h)$ no corolário anterior, define naturalınente um subgrupo próprio do grupo de contato $\mathcal{K}$. Mais precisamente, para cadla par $(M, h)$, podemos considerar o difeomorfismo $H(x, y)=(h(x), M(x) \cdot y)$ e portanto, neste caso, $\theta_{x}(y)$ é linear para todo $x$. Embora os grupos em questão sejam distintos, o Corolário 1.46 mostra que as órbitas são as mesmas.

Exemplo 1.47. Os germes $f(x, y)=\left(x-y, x^{3}+y^{3}+3 x^{2} y+3 x y^{2}-y^{2}+x y\right) e g(x, y)=$ $\left(x, y^{3}\right)$ são $\mathcal{K}$-equivalentes. De fato, considere $M=\left(\begin{array}{ll}1 & 0 \\ y & 1\end{array}\right)$ e $h(x, y)=(x-y, x+y)$. É fácil ver que $M$ é uma matriz $2 \times 2$ inversivel, pois $\operatorname{det} M(0) \neq 0, h$ é um difeomorfismo do $\mathbb{R}^{2}$ e $f=M \cdot g \circ h$.

Exemplo 1.48. Os germes $f(x)=\left(x^{2}, x^{3}\right)$ e $g(x)=\left(x^{2}, 0\right)$ são $\mathcal{K}$-erquivalentes, pois considerando $h(x)=x$ temos que $\langle f\rangle=\left\langle x^{2}\right\rangle=\langle g\rangle=\langle g \circ h\rangle$.

Outro conceito importante em teoria de singularidades é o conceito de desdobramento (para maiores detalhes ver [32], |65|). Em geral, a teoria de desdobramentos está relacionada com as aplicaçôes da teoria de singularidades.

Definiçāo 1.49. Seja $f:\left(\mathbb{R}^{n}, 0\right) \rightarrow\left(\mathbb{R}^{p}, 0\right)$. Um desdobramento de $f$ com $r$ parâmetros é um germe

$$
\begin{aligned}
F:\left(\mathbb{R}^{n} \times \mathbb{R}^{r}, 0\right) & \rightarrow\left(\mathbb{R}^{\mu} \times \mathbb{R}^{r}, 0\right) \\
(x, u) & \mapsto(\bar{f}(x, u), u)
\end{aligned}
$$

onde $\tilde{f}:\left(\mathbb{R}^{n} \times \mathbb{R}^{r}, 0\right) \rightarrow\left(\mathbb{R}^{p}, 0\right)$ é tal que $\tilde{f}(x, 0)=f(x)$. O germe $\tilde{f}$ é chamado de deformação de $f$.

Sejam $f \in \mathcal{E}_{n, p}$ e $W \subset \mathcal{E}_{n, p}$ um espaço vetorial real de dimensão $r$. Suponha que $\mathcal{E}_{n, p} \cong T_{f} \mathcal{K} \oplus W$ e sejam $\phi_{1}, \ldots, \phi_{r}$ uma base para $W$. Eintão,

$$
F(x, u)=f(x)+\sum_{i=1}^{r} u_{i} \phi_{i}, \quad \text { com } u=\left(u_{1}, \ldots, u_{r}\right)
$$

é um desdobramento de $\int$ com um número mínimo de parâmetros. Isto é consequência do teorema fundamental da teoria de desdobramentos $(\mid 32],[65])$. 
Para apresentarmos a caracterização geométrica da $\mathcal{K}$-equivalência introduzida por Montaldi [39], considere $\mathcal{X}_{i}$ e $\mathcal{Y}_{i}, i=1,2$, subvariedades do $\mathbb{R}^{n}$, com dim $\mathcal{X}_{1}$ - $\operatorname{dim} \mathcal{X}_{2}$ e $\operatorname{dim} \mathcal{Y}_{1}=\operatorname{dim} \mathcal{Y}_{2}$

Definição 1.50. Dizemos que o par $\left(\mathcal{X}_{1}, \mathcal{Y}_{1}\right)$ em $y_{1} \in \mathcal{X}_{1} \cap \mathcal{Y}_{1}$ tem o mesmo tipo de contato que o par $\left(\mathcal{X}_{2}, \mathcal{Y}_{2}\right)$ em $y_{2} \in \mathcal{X}_{2} \cap \mathcal{Y}_{2}$ se existe um germe de difeomorfismo $H:\left(\mathbb{R}^{n}, y_{1}\right) \longrightarrow\left(\mathbb{R}^{n}, y_{2}\right)$ tal que $H\left(\mathcal{X}_{1}\right)=\mathcal{X}_{2}$ e $I\left(\mathcal{Y}_{1}\right)=\mathcal{Y}_{2}$.

Notação: $K\left(\mathcal{X}_{1}, \mathcal{Y}_{1}, y_{1}\right)=K\left(\mathcal{X}_{2}, \mathcal{Y}_{2}, y_{2}\right)$.

Teorema 1.51. (Montaldi [39]) Sejam $g_{i}:\left(\mathcal{X}_{i}, 0\right) \longrightarrow\left(\mathbb{R}^{n}, 0\right)$ germes de imersões e $f_{i}:\left(\mathbb{R}^{n}, 0\right) \longrightarrow\left(\mathbb{R}^{p}, 0\right)$ germes de submersões com $f_{i}^{-1}(0)=\mathcal{Y}_{i}, i=1$, 2. Então, os germes $f_{1} \circ g_{1}$ e $f_{2} \circ g_{2}$ säo $\mathcal{K}$-equivalentes se, e somente se, $K\left(\mathcal{X}_{1}, \mathcal{Y}_{1}, 0\right)=K\left(\mathcal{X}_{2}, \mathcal{Y}_{2}, 0\right)$.

Com este resultado Montaldi [39] generaliza a idéia geométrica de contato proposta por Mather [33]:

Corolário 1.52. (Mather [39]) Sejam $f, g:\left(\mathbb{R}^{n}, 0\right) \longrightarrow\left(\mathbb{R}^{n}, 0\right)$. Entâo, os germes $f$ e $g$ são $\mathcal{K}$-equivalentes se, e somente se, graf $(f)$ e o graf $(g)$ têm o mesmo contato com o $\mathbb{R}^{n}$ na origem (isto é, $\left.K\left(\operatorname{graf}(f), \mathbb{R}^{n}, 0\right)=K\left(\operatorname{graf}(g), \mathbb{R}^{n}, 0\right)\right)$.

Montaldi também mostra que a álgebra local de contato

$$
Q(\mathcal{X}, \mathcal{Y}, y)=\frac{\mathcal{E}_{n}}{I(\mathcal{X})+I(\mathcal{Y})}
$$

onde $I(\mathcal{X})$ (resp. $I(\mathcal{Y}))$ ć o ideal de $\mathcal{E}_{n}$ dos germes que se anulam em $\mathcal{X}$ (resp. em $\left.\mathcal{Y}\right)$, um invariante completo do tipo de contato entre pares de variedades, quando o contato é do tipo finito, isto é,

$$
K\left(\mathcal{X}_{1}, \mathcal{Y}_{1}, y_{1}\right)=K\left(\mathcal{X}_{2}, \mathcal{Y}_{2}, y_{2}\right) \Leftrightarrow Q\left(\mathcal{X}_{1}, \mathcal{Y}_{1}, y_{1}\right) \text { ○ } Q\left(\mathcal{X}_{2}, \mathcal{Y}_{2}, y_{2}\right)
$$

são isomorfos induzidos (ver [39]). 


\section{Capítulo 2}

\section{Equivalência de contato topológica $\left(\mathcal{C}^{0}-\mathcal{K}\right.$-equivalência)}

Conforme vimos no capítulo anterior, os Teoremas 1.43 e 1.44 fornecem invariantes para a $\mathcal{K}$-equivalência. Entretanto, existe moduli para as $\mathcal{K}$-órbitas. Assim, parece natural introduzir a versão topológica da $\mathcal{K}$-equivalência e também verificar versões topológicas destes invariantes. Outra motivação para o estudo da $\mathcal{C}^{0}-\mathcal{K}$-equivalência é a sua relação com a $\mathcal{C}^{1}$ - $\mathcal{V}$-equivalência e a $\mathcal{C}^{0}$ - $\mathcal{A}$-cquivalência, conforme veremos na Seção 2.1 .

Definição 2.1. Dizemos que dois germes $f, g:\left(\mathbb{R}^{n}, 0\right) \longrightarrow\left(\mathbb{R}^{p}, 0\right)$ são topologicamente $\mathcal{K}$-equivalentes (ou $\mathcal{C}^{0}$-K-equivalentes) se existem germes de homeomorfismos $h:\left(\mathbb{R}^{n}, 0\right) \longrightarrow\left(\mathbb{R}^{n}, 0\right)$ e $H:\left(\mathbb{R}^{n} \times \mathbb{R}^{p}, 0\right) \longrightarrow\left(\mathbb{R}^{n} \times \mathbb{R}^{p}, 0\right)$ satisfazendo a propriedade $H\left(\mathbb{R}^{n} \times\{0\}\right)=\mathbb{R}^{n} \times\{0\}$ e tais que os seguintes diagramas comutam

$$
\begin{aligned}
& \left(\mathbb{R}^{n}, 0\right) \stackrel{(i d, f)}{\longrightarrow}\left(\mathbb{R}^{n} \times \mathbb{R}^{p}, 0\right) \stackrel{\pi_{n}}{\longrightarrow}\left(\mathbb{R}^{n}, 0\right) \\
& h \downarrow \quad H \downarrow \quad h \downarrow \\
& \left(\mathbb{R}^{n}, 0\right) \stackrel{\left(i d_{, g}\right)}{\longrightarrow}\left(\mathbb{R}^{n} \times \mathbb{R}^{p}, 0\right) \stackrel{\pi_{n}}{\longrightarrow}\left(\mathbb{R}^{n}, 0\right)
\end{aligned}
$$

onde id $:\left(\mathbb{R}^{n}, 0\right) \rightarrow\left(\mathbb{R}^{n}, 0\right)$ é a aplicação identidade do $\mathbb{R}^{n}$ e $\pi_{n}:\left(\mathbb{R}^{n} \times \mathbb{R}^{p}, 0\right) \rightarrow\left(\mathbb{R}^{n}, 0\right)$ a proję̧ão canônica.

Em outras palavras, $H$ é dado por $H(x, y)=(h(x), \theta(x, y))$, com $\theta(x, 0)=0 . \quad O$ homeomorfismo $H$ aplica o gráfico de $f$ no gráfico de $g$, enquanto h atua em $\mathbb{R}^{n}$.

Em particular, definimos a $\mathcal{C}^{0}$-C-equivalência quando $h=i d$.

Notaçào. $f \stackrel{\mathcal{L}^{0}-\mathcal{K}}{\sim} g$.

Note que esta relação de equivalência é a versão topológica da $\mathcal{K}$-equivalência de Mather dada na Definição 1.37. Basta substituirmos difeomorfismos por homeomorfismos. Procedendo da mesma maneira, podemos obter as outras versões topológicas para a 
Definição 1.37, a saber: a $\mathcal{C}^{0}-\mathcal{R}, \mathcal{C}^{0}-\mathcal{L}$ e $\mathcal{C}^{0}-\mathcal{A}$ equivalências. A $\mathcal{C}^{0}$ - $\mathcal{A}$-equivalência é apresentadia muitas vezes na literatura como equivalência topológica (ver [44], por exemplo).

Exemplo 2.2. Os germes de funçôes em uma variável real $f(x)=x^{3}$ e $g(x)=x$ são $\mathcal{C}^{0}-\mathcal{K}$-equivalentes. De fato, basta considerar os homeomorfismos $h=i d:(\mathbb{R}, 0) \longrightarrow(\mathbb{R}, 0)$ e $H:(\mathbb{R} \times \mathbb{R},(0,0)) \longrightarrow(\mathbb{R} \times \mathbb{R},(0,0))$ dado por $H(x, y)=\left(x, y^{3}\right)$. Na verdade estes germes são $\mathcal{C}^{0}-\mathcal{C}$-equivalentes. Observe que $f$ e g não são $\mathcal{K}$-equivalentes (Teorema 1.43 ).

Do mesmo modo como antes, continuamos interessados na classe dos germes que podem ser representados pelo seu polinômio de Taylor de alguma ordem. Assim, introduzimos o conceito de $\mathcal{C}^{0}-\mathcal{K}$-determinação finita. No caso complexo, Wall [65] provor que germes $\mathcal{C}^{0}-\mathcal{K}$-finitos são também $\mathcal{K}$-finitos. Mas a classe dos germes $\mathcal{C}^{0}-\mathcal{K}$-finitos contém estritamente a classe dos germes $\mathcal{K}$-finitos, no caso real. Por isso, em muitos resultados adotamos a $\mathcal{C}^{0}-\mathcal{K}$-determinação finita para classificar nossos objetos. Os aspectos básicos da teoria de singularidades que dizem respeito ao estudo da $\mathcal{C}^{0}-\mathcal{K}$-determinação são bem conhecidos e para maiores detalhes citamos como referência [65].

Definição 2.3. Um germe $f:\left(\mathbb{R}^{n}, 0\right) \longrightarrow\left(\mathbb{R}^{p}, 0\right)$ é $r-\mathcal{C}^{0}-\mathcal{K}$-determinado se para todo germe $g:\left(\mathbb{R}^{n}, 0\right) \longrightarrow\left(\mathbb{R}^{p}, 0\right)$ com $j^{r} f(0)=j^{r} g(0)$ então f é $\mathcal{C}^{0}-\mathcal{K}$-equivalente a $g$.

$S e f$ é $r-\mathcal{C}^{0}-\mathcal{K}$-determinado para algum $r$, então $f$ é $\mathcal{C}^{0}-\mathcal{K}$-finitamente determinado ou $\mathcal{C}^{0}-\mathcal{K}$-finito er (o menor possível) é o grau de determinação de $f$.

O teorema a seguir é devido a du Plessis e apresenta uma estimativa para o grau de $\mathcal{C}^{0}-\mathcal{K}$-determinação.

Teorema 2.4. ([65]) Sejam $f$ um germe de aplicação e I um ideal eliptico em $\mathcal{E}_{n}$ (isto é, $\left.I \supset m_{n}^{\infty}\right)$ satisfazendo

$$
\operatorname{Im} m_{n}^{r} \theta_{f} \subset I T_{f} \mathcal{K}
$$

Então, $f$ ér $-\mathcal{C}^{0}-\mathcal{K}$-determinado.

Outra propriedade interessante dos germes $\mathcal{C}^{0}-\mathcal{K}$-finitos é a seguinte:

Proposição 2.5. ([57]) Seja $f:\left(\mathbb{R}^{n}, 0\right) \rightarrow\left(\mathbb{R}^{p}, 0\right), n>p$, um germe $r-\mathcal{C}^{0}$-K -determinado. Então, existe um germe $g$, com $j^{r} g(0)=j^{r} f(0), \mathcal{K}$-finito e $\mathcal{C}^{0}-\mathcal{K}$-equivalente a $f$.

Como consequência da proposição acima, segue que uma variedade real definida por um germe $\mathcal{C}^{0}$-K-finito é topologicamente equivalente a uma variedade definida por um germe 
$\mathcal{K}$-finito, ou seja, existe um homeomorfismo $h:\left(\mathbb{R}^{n}, 0\right) \rightarrow\left(\mathbb{R}^{n}, 0\right)$ tal que $h\left(f^{-1}(0)\right)=$ $g^{-1}(0)$

Um critério geométrico para a $\mathcal{C}^{0}-\mathcal{K}$-determinação finita de germes de aplicação analítica real é dado por:

Proposição 2.6. (l65]) O germe $f e^{0} \mathcal{C}^{0}-\mathcal{K}$-finitamente determinado se, e somente se, $\left(f^{\cdots 1}(0) \cap \Sigma f\right)-\{0\}=\emptyset$ como germes.

Observação. A implicaçăo

$$
\mathcal{C}^{(1)} \text { - K-finitamente determinado } \Rightarrow\left(f^{-1}(0) \cap \Sigma \int\right)-\{0\}=\emptyset
$$

é sempre verdadeira, mesmo o germe não sendo analítico.

Ao contrário do que ocorre com o grupo $\mathcal{K}$, a propriedade de $\mathcal{C}^{(1)} \mathcal{K}$-determinação finita não é neccssariamente aberta, isto é, deformações arbitrariamente próximas de um germe $\mathcal{C}^{0}-\mathcal{K}$-finitamente determinado podem não ser $\mathcal{C}^{0}$ - $\mathcal{K}$-finitannente determinadas. Por exemplo, considere o germe $f:\left(\mathbb{R}^{2}, 0\right) \rightarrow(\mathbb{R}, 0)$ dado por

$$
f(x, y)=\left(x^{2}+y^{2}\right)^{2}
$$

Como $f^{-1}(0)=\{0\},\left(f^{-1}(0) \cap \Sigma f\right)-\{0\}=\emptyset \mathrm{e}$, portanto, $\mathcal{C}^{0}$ - $\mathcal{K}$-finitamente determinado. Mas a deformação de $f$ dada por $f_{\varepsilon}(x, y)=\left(x^{2}+y^{2}-\varepsilon x\right)^{2}$ não é $\mathcal{C}^{0}$ - $\mathcal{K}$-finitamente determinada para $\varepsilon$ próximo de zero, pois $\left(f_{\varepsilon}^{-1}(0) \cap \Sigma f_{\varepsilon}\right)-\{0\} \neq \emptyset$.

Dada $f:\left(\mathbb{R}^{n}, 0\right) \longrightarrow\left(\mathbb{R}^{p}, 0\right)$, seja $N_{\mathcal{C}} f=|f(x)|^{2}, N_{\mathcal{R}} f=\sum_{j} M_{j}^{2}$, onde os $M_{j}$ são os geradores do ideal de $\mathcal{E}_{n}$ gerado pelos $p \times p$-menores da matriz jacobiana de $f \mathrm{e}$ $N_{\mathcal{K}} f=N_{\mathcal{C}} f+N_{\mathcal{R}} f$. Dizemos que $N_{\mathcal{K}} f$ salisfaz uma condiçào de Lojasicuicz se existem constantes $c>0$ e $\alpha>0$ tais que $N_{\mathcal{K}} f(x) \geq c|x|^{\alpha}$.

Proposiçāo 2.7. ([65]) $N_{\mathcal{K}} f(x)$ satisfuz uma condição de Lojasieuicz se, e somente se, $f$ é $\mathcal{C}^{0}-\mathcal{K}$-finitamente determinado.

O seguinte lema relaciona a existência de uma condição de Lojasiewicz para $\phi=\sum_{i} \phi_{i}^{2}$ com as condições para que o ideal gerado pelas $\phi_{i}$ 's scja elíptico.

Lema 2.8. ([65]) Seja $I=\left\langle\phi_{1}, \ldots, \phi_{t}\right\rangle$ um ideal finitumente gerado de $\mathcal{E}_{n}$. Então, as seguintes condições säo equivalentes:

a) $I \supset m_{n}^{\infty}(I$ é elíptico)

b) (Condiçäo de Lojasiewicz) Existe uma função $f$, definida em uma vizinhança $U$ de 0 , com germe em $I$, tal que $|f(x)| \geq c|x|^{\alpha}$ em $U$ para algum $c>0, \alpha>0$. 
c) Alguma desigualdade $\sum_{i=1}^{t}\left(\phi_{i}(x)\right)^{2} \geq c|x|^{\alpha}$ ocorre em alguma vizinhança de 0 .

Além disso, se as $\phi_{i}$ são analíticas, as condições acima são equinulentes a

d) O é ponto isolado no conjunto $\left\{x \in\left(\mathbb{R}^{n}, 0\right) \mid \phi_{1}(x)=\ldots=\phi_{t}(x)=0\right\}$.

Teorema 2.9. ([65]) São equivalentes:

a) $f$ é $\mathcal{C}^{0}-\mathcal{K}$-finitamente determinado;

b) $T_{f} \mathcal{K} \supseteq m_{n}^{\infty} \theta_{f}$;

c) o ideal $I_{\mathcal{K}}(f)=J_{f}+f^{\star}\left(m_{p}\right) \theta_{f}$ é elíptico, onde $J_{f}$ é o ideal de $\mathcal{E}_{n}$ gerado pelos $p \times p$-menores da matriz jacobiana de $f$.

Alguns invariantes conhecidos para a $\mathcal{K}$-equivalência, como a $\mathcal{K}$-codimensão c os símbolos de Boardman (ver [23]), não são invariantes para a $\mathcal{C}^{0}-\mathcal{K}$-equivalĉncia. Além disso, outros possíveis candidatos como, por exemplo, $\mathbb{R}$-álgebra local, a multiplicidade e a $\mathcal{C}^{0}-\mathcal{K}$-determinação finita, também não são invariantes para a $\mathcal{C}^{0}-\mathcal{K}$-equivalência. Veja os exemplos a seguir.

Exemplo 2.10. Considere os germes $f(x)=x$ e $g(x)=x^{3}$. Vimos no Exemplo 2.2 que $f$ e g são $\mathcal{C}^{0}-\mathcal{K}$-equivalentes. Claramente as $\mathbb{R}$-álgebras locais $Q(f)$ e $Q(g)$ não são isomorfas, pois apresentam dimensões diferentes como $\mathbb{R}$-espaços vetoriais. Além disso, estes germes têm multiplicidades diferentes e pela Definição 1.38, as $\mathcal{K}$-codimensões também são diferentes. Logo estes objetos não são invariantes para a $\mathcal{C}^{0}-\mathcal{K}$-equivalência.

Exemplo 2.11. Considere $f(x, y)=x y$ e $g(x, y)=x y^{3}$. Estes germes são $\mathcal{C}^{0}$ - $\mathcal{A}$-equivalentes (e portanto, $\mathcal{C}^{0}-\mathcal{K}$-equivalentes). Além disso, $f$ é $\mathcal{C}^{0}-\mathcal{K}$-finito mas o mesmo não ocorre com g. Portanto, a $\mathcal{C}^{0}-\mathcal{K}$-determinação finita não é urm invariante para a $\mathcal{C}^{0}-\mathcal{K}$ equivalência.

Exemplo 2.12. O símbolo de Boardman também não é um invariante da $\mathcal{C}^{0}-\mathcal{K}$-equivalência. De fato, novamente considere os germes $f(x)=x$ e $g(x)=x^{3}$. Estes germes têm símbolo de Boardman distintos, a saber $(0 ; 0)$ e $(1,1 ; 0)$, respectivamente (ver [23], $p$. 180).

Exemplo 2.13. A propriedade do germe ser não singular, não é um invariante para a $\mathcal{C}^{0}-\mathcal{K}$-equivalência. De fato, considere os germes $f, g:\left(\mathbb{R}^{3}, 0\right) \rightarrow\left(\mathbb{R}^{2}, 0\right)$, dados por

$$
f(x, y, z)=(x, y) \text { e } g(x, y, z)=\left(x, y^{3}\right) .
$$


O germe $f$ é näo singular, $f$ eg são $\mathcal{C}^{0}-\mathcal{K}$-equivalentes, mas g é singular.

No caso complexo, esta propriedade é um invariante para a $\mathcal{C}^{0}$-A-equivalência (ver 144]).

Além da dificuldade de encontrar invariantes, outro problema ć a falta de caracterizações para a $\mathcal{C}^{0}-\mathcal{K}$-equivalência. Por excmplo, suponha que estivéssemos interessados em uma versão topológica para Proposição $1.45, a) \Leftrightarrow c)$. Entrão, se considerarmos novamente os germes $\mathcal{C}^{0}$ - $\mathcal{C}$-equivalentes $f(x)=x$ e $g(x)=x^{3}$, precisaríamos dar condiçôes a umn germe de matriz $M, 1 \times 1$, satisfazendo algo do tipo

$$
f=M \cdot g
$$

Assim, se de forma mais natural, assumirmos $M$ inversível com entradas contínuas, deveríamos ter $M(0) \neq 0$ e poderíamos escrever $M(x)=a(x)+c$, onde $a$ é uma função contínua com $a(0)=0$ e $c$ uma constante real não nula. Ficaríamos então com

$$
x^{3}=(a(x)+c) x \Leftrightarrow x\left(x^{2}-c\right)=a(x) x .
$$

Fazendo $x$ tender a 0 , segue da expressão (2.1) que

$$
0 \neq-c=\lim _{x \rightarrow 0} \frac{x\left(x^{2}-c\right)}{x}=\lim _{x \rightarrow 0} a(x)=0,
$$

o que o um absurdo.

Portanto, supondo $M$ inversível com entradas contínuas, não obtemos uma versão topológica da Proposição 1.45 .

Como podemos notar, os resultados com as propriedades e invariantes para a $\mathcal{C}^{0}-\mathcal{K}$ equivalência são incompletos. Na verdade não cxistcrm caracterizações algébricas ou geométricas. Além disso, o único invariante completo que conhecemos para $\mathcal{C}^{0}-\mathcal{K}$-equivalência co valor absoluto do grau, introduzido por Nishimura em [45], para o caso $n=p$.

\subsection{Resultados de Nishimura e o caso $n=p$}

Para obter o valor absoluto do grau como invariante, Nishimura utiliza o Lema 2.14 a seguir. Este lema fornece uma condição suficiente para a $\mathcal{C}^{0}$ - $\mathcal{K}$-equivalência e ć umas das principais ferramentas deste nosso trabalho.

Lema 2.14. (Nishimura /45]) Sejam $f, g: U \longrightarrow \mathbb{R}^{p}$ aplicaçôes contínuas onde $U$ é uma vizinhança da origem em $\mathbb{R}^{n}$. Suponha que exista uma fumília de aplicações contínuas $F_{t}: U \longrightarrow \mathbb{R}^{p}, t \in[0,1]$, tal que as seguintes condições são satisfeitas 
i) $F_{0}=f$ e $F_{1}=g$ ou $g=\left(g_{1}, \ldots, g_{p-1},-g_{p}\right)$;

ii) $F_{t}^{-1}(0)=f^{1}(0)$, para todo $t \in[0,1]$;

iii) Para qualquer $t \in[0,1]$, o vetor $F_{t}(x)$ nãu pertence ao conjunto $\left\{\alpha F_{0}(x) \mid \alpha<0\right\}$, para qualquer $x \in U-f^{-1}(0)$.

Então, $F_{t}$ é $\mathcal{C}^{0}-\mathcal{K}$-equivalente a $F_{t}^{\prime}$, quaisquer que sejam $t, t^{\prime} \in[0,1]$. Em particular, $f$ é $\mathcal{C}^{0}-\mathcal{K}$-equivalente a $g$.

Prova. Fixe $t \in[0,1]$ qualquer. Vamos mostrar que $F_{t} \dot{c} \mathcal{C}^{0}-\mathcal{K}$-equivalente a $f=F_{0}$. De fato, considere uma família de homeomorfismos $\tilde{h}_{x}: \mathbb{R}^{p} \longrightarrow \mathbb{R}^{p}$ definida por

$$
\tilde{h}_{x}(y)=\alpha_{x}(\|y\|) \cdot y
$$

dependendo continuamente da variável $x$ de $U$, onde $\alpha_{x}: \mathbb{R}_{+} \longrightarrow \mathbb{R}$ é uma função contínua dependendo de $\|y\|(\neq 0)$ conforme apresentaremos a seguir.

Note que ao compararmos $\left\|F_{0}(x)\right\|$ com $\left\|F_{t}(x)\right\|$ para $x \in U$ temos três possibilidades:

I.) $\left\|F_{0}(x)\right\|=\left\|F_{t}(x)\right\|$.

Neste caso tome $\alpha_{x}(\|y\|)=1$ para todo $y \in \mathbb{R}^{p}-\{0\}$.

Observe que o caso $x \in F_{0}^{-1}(0)=F_{t}^{-1}(0)$ está incluído aqui.

II.) $0<\left\|F_{t}(x)\right\|<\left\|F_{0}(x)\right\|$.

Neste caso tome $\alpha_{x}$ definida por:

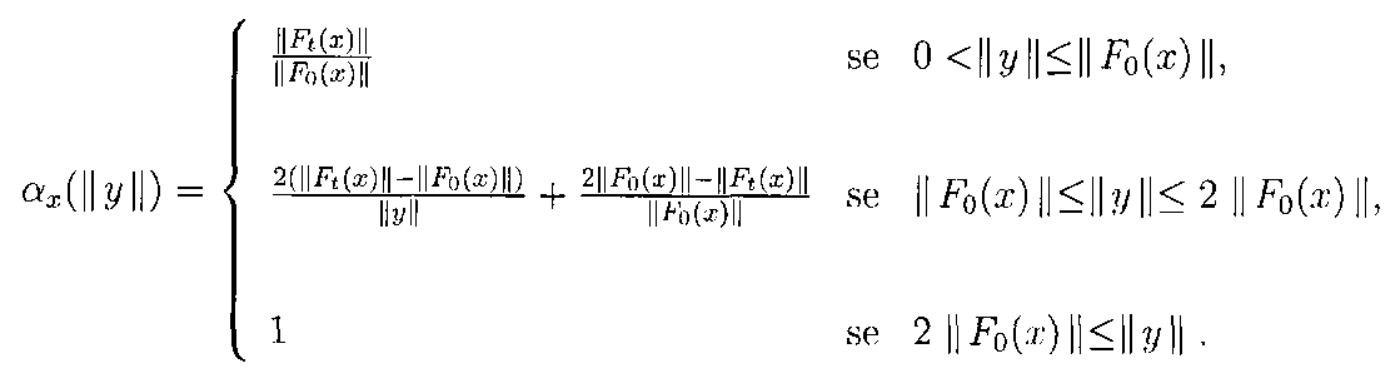

III.) $0<\left\|F_{0}(x)\right\|<\left\|F_{t}(x)\right\|$.

Neste caso tome $\alpha_{x}$ definida por:

$\alpha_{x}(\|y\|)= \begin{cases}\frac{\left\|F_{t}(x)\right\|}{\left\|F_{0}(x)\right\|} & \text { se } 0<\|y\| \leq\left\|F_{0}(x)\right\|, \\ \frac{2\left\|F_{t}(x)\right\|\left(\left\|F_{t}(x)\right\|-\left\|F_{0}(x)\right\|\right)}{2\left\|F_{t}(x)\right\|-\cdots F_{0}(x) \|} \frac{1}{\|y\|}+\frac{\left\|F_{i}(x)\right\|}{2\left\|F_{t}(x)\right\|-\left\|F_{0}^{\prime}(x)\right\|} & \text { se }\left\|F_{0}(x)\right\| \leq\|y\| \leq 2\left\|F_{t}(x)\right\|, \\ 1 & \text { se } 2\left\|F_{t}(x)\right\| \leq\|y\| .\end{cases}$ 
Se definimos a função $\alpha_{x}$ como acima, então $\tilde{h}_{x}$ é um homeomorfismo para qualquer $x \in U$. Além disso, $\tilde{h}_{x}$ depende continuamente de $x \in \mathbb{R}^{n}$.

Construa outra família de homcomorfismos $\bar{h}_{x}: \mathbb{R}^{p} \longrightarrow \mathbb{R}^{p}$ dependendo continuamente da variável $x \in U$. De acordo com a relação entre $\tilde{h}_{x}\left(F_{0}(x)\right)$ e $F_{l}(x)$ para $x \in U$, temos dois casos.

I.) $\tilde{h}_{x}\left(F_{0}(x)\right)=F_{t}(x)$.

Neste caso defina $\bar{h}_{x}$ como a aplicação identidade.

II.) $\tilde{h}_{x}\left(F_{0}(x)\right) \neq F_{t}(x)$.

Neste caso, observe que $\left\|\tilde{h}_{x}\left(F_{0}(x)\right)\right\|=\left\|F_{t}(x)\right\|>0$, pela construção de $\tilde{h}_{x}$.

Pela condição iii) da hipótese sobre a família $F_{t}$ e pela construção de $\tilde{h}_{x}$, segue que os dois vetores $\tilde{h}_{x}\left(F_{0}(x)\right)$ e $F_{t}(x)$ de $\mathbb{R}^{p}$ são linearmente independentes sobre $\mathbb{R}$. Considere o espaço vetorial

$$
V_{x}=\mathbb{R} \tilde{h}_{x}\left(F_{0}(x)\right) \oplus \mathbb{R} F_{t}(x) .
$$

Seja $\theta_{x}$ o ângulo entre $\tilde{h}_{x}\left(F_{0}(x)\right)$ e $F_{t}(x)$, o qual é unicamente determinado no intervalo aberto $(0, \pi)$. Usando este ângulo $\theta_{x}$, construímos uma função ângulo $\Theta_{x}: \mathbb{R}_{+} \cup\{0\} \rightarrow \mathbb{R}$ da seguinte forma.

$$
\Theta_{x}(s)= \begin{cases}\theta_{x} & \text { se } 0 \leq s \leq\left\|F_{t}(x)\right\|, \\ -\frac{\theta_{x}}{\left\|F_{t}(x)\right\|} s+2 \theta_{x} & \text { se }\left\|F_{t}(x)\right\| \leq s \leq 2\left\|F_{t}(x)\right\|, \\ 0 & \text { se } 2\left\|F_{t}(x)\right\| \leq s .\end{cases}
$$

Decomponha $\mathbb{R}^{p}$ na soma direta

$$
\mathbb{R}^{p}=V_{x} \oplus \tilde{V}_{: x}
$$

Considere no espaço vetorial bi-dimensional $V_{x}$ a orientação padrão induzida pelo $\mathbb{R}^{2}$ através do isomorfismo linear $L: \mathbb{R}^{2} \rightarrow V_{x}$ tal que $L\left(\overrightarrow{e_{1}}\right)=\tilde{h}_{x}\left(F_{0}(x)\right)$ e $L\left(\overrightarrow{e_{2}}\right)=F_{t}(x)$, onde $L\left(\overrightarrow{e_{1}}\right)$ e $L\left(\overrightarrow{e_{2}}\right)$ são os vetores da basc canônica de $\mathbb{R}^{2}$. Usando a função ângulo $\Theta_{x}$, construímos a aplicação $\bar{h}_{x}: V_{x} \oplus \tilde{V}_{x}$ como segue:

$$
\bar{h}_{x}\left(y_{1}, y_{2}\right)=\left(\phi_{\Theta_{x}\left(\| y_{1} \mid\right)}\left(y_{1}\right), y_{2}\right),
$$

onde $\phi_{\left.\Theta_{x}:|| y_{1}||\right)}$ é a aplicação rotação de ângulo $\Theta_{x}\left(\left\|y_{1}\right\|\right)$,

$$
\phi_{\Theta_{x}\left(\left\|y_{y}\right\|\right)}=\left(\begin{array}{rr}
\cos \left(\Theta_{x}\left(\left\|y_{1}\right\|\right)\right) & -\sin \left(\Theta_{x}\left(\left\|y_{1}\right\|\right)\right) \\
\sin \left(\Theta_{x}\left(\left\|y_{1}\right\|\right)\right) & \cos \left(\Theta_{x}\left(\left\|y_{1}\right\|\right)\right)
\end{array}\right) .
$$


$\bar{h}_{x}$ é um homeomorfismo para qualquer $x \in U$ e depende continuamente de $x$.

Portanto, $\ddot{h}_{x} \circ \tilde{h}_{x}\left(F_{0}(x)\right)=F_{t}(x)$ para qualquer $x \in U$. Defina então a aplicação $H: U \times \mathbb{R}^{p} \longrightarrow U \times \mathbb{R}^{p}$ por $H(x, y)=\left(x, \ddot{h}_{x} \circ \tilde{h}_{x}(y)\right)$.

Por construçẫo, $H$ é bijetiva e contínua. Pelo Teorema da Invariância do Domínio segue que $H$ é um homeomorfismo. Assim, o par $(i d, H)$ satisfaz as condições da Definição 2.1 fornecendo a $\mathcal{C}^{0}-\mathcal{K}$-equivalência entre $F_{0}=\int$ e $F_{t}$, para o $t$ fixo desde o início. Portanto, $F_{t}$ e $F_{t}^{\prime}$ são $\mathcal{C}^{0}-\mathcal{K}$-equivalentes, quaisquer que sejam $t, t^{\prime} \in[0,1]$.

Como $g$ e $\vec{g}=\left(g_{1}, \ldots, g_{p-1},-g_{p}\right)$ são trivialmente $\mathcal{L}$-equivalentes, então podemos concluir que $f$ e $g$ são $\mathcal{C}^{0}-\mathcal{K}$-equivalentes.

Definição 2.15. Dizemos que dois germes $f, g:\left(\mathbb{R}^{n}, 0\right) \longrightarrow\left(\mathbb{R}^{p}, 0\right)$ são topologicamente $\mathcal{V}$-equivalentes (ou $\mathcal{C}^{0}$ - $\mathcal{-}$-equivalentes) se existe um germe de homeomorfismo $h:\left(\mathbb{R}^{n}, 0\right) \longrightarrow\left(\mathbb{R}^{n}, 0\right)$ tal que $h\left(f^{-1}(0)\right)=g^{-1}(0)$.

É fácil ver que $\mathcal{C}^{0}-\mathcal{K}$-equivalência implica $\mathcal{C}^{0}-\mathcal{V}$-equivalência. Segue do Lema 2.14 o seguinte corolário:

Corolário 2.16. (Nishimura [45]) Sejam $f, g:\left(\mathbb{R}^{n}, 0\right) \longrightarrow(\mathbb{R}, 0)(n \geq 2)$ germes de funçōes $\mathcal{C}^{0}-\mathcal{K}$-finitamente determinados. Então, $f$ eg são $\mathcal{C}^{0}-\mathcal{K}$-equivalentes se, e somente se, $f$ e $g$ são $\mathcal{C}^{0}-\mathcal{V}$-equivalentes.

Observação. Por cxemplo, sc considerarmos $f(x, y)=x+y$ e $g(x, y)=(x+y)^{2}$, cmbora estes germes tenham o mesmo conjunto de zeros, o Corolário 2.16 não diz se eles são $\mathcal{C}^{0}-\mathcal{K}$-equivalentes, pois $g$ não é $\mathcal{C}^{0}-\mathcal{K}$-finito. No Capítulo 4 apresentamos um invariante pclo qual podemos ver que estes germes não são $\mathcal{C}^{0}-\mathcal{K}$-equivalentes.

Outro ponto interessante que motiva o estudo da $\mathcal{C}^{0}-\mathcal{K}$-equivalência é a sua relação com a $\mathcal{C}^{0}-\mathcal{V}$-equivalência. Pelo Corolário 2.16 temos que, no caso de funçõos reais $\mathcal{C}^{0}-\mathcal{K}$ finitamente determinadas, estas definiçôes são equivalentes. Mas isto não vale, em geral, para aplicações. De fato, considere os germes $f(x, y)=(x, y)$ e $g(x, y)=\left(x^{2}-y^{2}, 2 x y\right)$ Estes germes são $\mathcal{C}^{0}-\mathcal{K}$-finitamente determinados pois $f^{-1}(0)=g^{-1}(0)=\{0\}$ e também $\mathcal{C}^{0}-\mathcal{V}$-equivalentes, mas não são $\mathcal{C}^{0}-\mathcal{K}$-equivalentess (será fácil ver isto usando o Teorema 2.17 a seguir).

Para germes de funções complexas, existe uma relação entre $\mathcal{C}^{0}-\mathcal{V}$-equivalência e $\mathcal{C}^{0}$ - $\mathcal{A}$-equivalência (ver [56]) mas, no caso de funções reais, temos vários contra-exemplos de que essas relações não são equivalentes (ver [27]). Assim, como a $\mathcal{C}^{0}$-K-equivalência é 
intermediária entre a $\mathcal{C}^{0}-\mathcal{A}$-equivalência e a $\mathcal{C}^{0}$ - $\mathcal{V}$-equivalência, é razoável tentar investigála. Uma outra razão é que, como sabemos que no caso complexo, $\mathcal{V}$-equivalência (isto é, substituindo homeomorfismo por difcomorfismo na Definição 2.15̃) implica $\mathcal{K}$-equivalência, para germes de aplicações $\mathcal{K}$-finitos com $n>p$ (ver [31]), não podemos esperar esta implicação no caso real. Então, no caso real, versões topológicas desta implicação poderiam ser investigadas.

Para o caso especial $n=p$, se um germe $\int$ é $\mathcal{C}^{0}$ - $\mathcal{K}$-finito então $f^{-1}(0)=\{0\}$, como germe. Sendo assim, é possível definir o grau da aplicação $f:\left(\mathbb{R}^{n}, 0\right) \rightarrow\left(\mathbb{R}^{n}, 0\right)$. Conforme veremos no tcorema a seguir, o valor absoluto do grau é um invariante completo para a $\mathcal{C}^{0}-\mathcal{K}$-equivalência.

Teorema 2.17. (Nishimura /45/) Sejam $f, g:\left(\mathbb{R}^{n}, 0\right) \longrightarrow\left(\mathbb{R}^{n}, 0\right)$ germes $\mathcal{C}^{0}-\mathcal{K}$-finitamente determinados e $n \neq 4$. Então,

$$
f \text { eg são } \mathcal{C}^{0}-\mathcal{K} \text {-equivalentes se, e somenle se, }|\operatorname{grau}(\mathrm{f})|=|\operatorname{grau}(\mathrm{g})| .
$$

O grau citado acima pode ser calculado como

$$
|\operatorname{grau}(\mathrm{f})|=\operatorname{dim}_{\mathbb{R}} \mathrm{Q}(\mathrm{f})-2 \operatorname{dim}_{\mathbb{R}} \mathrm{I},
$$

onde $I$ é um ideal de $Q(f)$ maximal com respeito a propriedade $I^{2}=0$.

Esta fórmula algébrica para o grau de um germe de aplicação $\mathcal{C}^{\infty}$ pode ser encontrada em [16]. Assim, usando a fórmula de Eisenbud-Levine [16], podemos recscrever o T'eorema 2.17 da seguinte forma:

Corolário 2.18. Sejam $f, g:\left(\mathbb{R}^{n}, 0\right) \longrightarrow\left(\mathbb{R}^{n}, 0\right)$ germes $\mathcal{C}^{0}-\mathcal{K}$-finitamente determinados e $n \neq 4$. Enlão, $\int$ eg são $\mathcal{C}^{0}-\mathcal{K}$-equivalentes se, e somenle se,

$$
\operatorname{dim}_{\mathbb{R}} Q(f)-2 \operatorname{dim}_{\mathbb{R}} I(f)=\operatorname{dim}_{\mathbb{R}} Q(g)-2 \operatorname{dim}_{\mathbb{R}} I(g),
$$

onde $I(h)$ é um ideal maximal da $\mathbb{R}$-álgebra local $Q(h)$ com respeito à propriedade $I^{2}=0$.

Por excmplo, quando $n=p=1, f(x)=x^{k}$ e $g(x)=x^{l}$ são $\mathcal{C}^{0}-\mathcal{K}$-equivalentes se, $\mathrm{e}$ somente se, $k$ e $l$ têm a mesma paridade.

Observação. A perspectiva da validade da prova de Perelman [49] para a Conjectura de Poincaré, pode eliminar a restrição $n \neq 4$ no Teorema 2.17. No artigo [45], Nishimura sugere que uma prova alternativa que inclua o caso $n=4$ talvez seja possível. Mas como o argumento para isto não parece fácil de ser provado, ele prefere omitir este caso.

A proposição a seguir garante que os germes $\mathcal{C}^{0}-\mathcal{K}$-equivalentes têm desdobramentos $\mathcal{C}^{0}-\mathcal{K}$-equivalentes. 
Proposição 2.19. (Nishimura [45]) Sejam $f, g:\left(\mathbb{R}^{n}, 0\right) \rightarrow\left(\mathbb{R}^{p}, 0\right)$ germes de aplicações continuas e sejam $F, G:\left(\mathbb{R}^{n} \times \mathbb{R}^{r}, 0\right) \rightarrow\left(\mathbb{R}^{p} \times \mathbb{R}^{r}, 0\right)$ desdobramentos de $f$ e g, respectivamente. Se $f$ eg são $\mathcal{C}^{0}-\mathcal{K}$-equivalentes então $F$ e $G$ são $\mathcal{C}^{0}-\mathcal{K}$-equivalentes.

A proposição acima também decorre do Lema 2.14 .

Para finalizar esta seção, completamos o nosso estudo da $\mathcal{C}^{0}-\mathcal{K}$-equivalência para o caso $n=p$, apresentando dois novos resultados: o Teorema 2.20 e a Proposição 2.21, conforme veremos a seguir.

Teorema 2.20. Seja $F_{t}:\left(\mathbb{R}^{n}, 0\right) \rightarrow\left(\mathbb{R}^{p}, 0\right), t \in[0,1]$, uma familia de germes $\mathcal{K}$-finitos, tais que $\operatorname{dim}_{\mathbb{R}} Q\left(F_{t}\right)$ é constante, para todo $t \in[0,1]$. Então, para quaisquer $t, t^{\prime} \in[0,1]$, $F_{l}$ e $F_{t^{\prime}}$ são $\mathcal{C}^{0}-\mathcal{K}$-equivalentes.

Prova. Observe que podemos supor $p=n$, pois para $p<n$ a dimensão da álgebra $Q\left(F_{t}\right)$ é sempre infinita e, então, o resultado não faz sentido, e para o caso $p>n$ segue do Teorema 3.6 que todos os germes são equivalentes.

Considerc cntão uma família $F_{\iota}:\left(\mathbb{R}^{n}, 0\right) \rightarrow\left(\mathbb{R}^{n}, 0\right), t \in[0,1]$, tal que $\operatorname{dim}_{\mathbb{R}} Q\left(F_{t}\right)$ é constante. Complexificando, obtemos $\left(F_{\mathbb{C}}\right)_{t}:\left(\mathbb{C}^{n}, 0\right) \rightarrow\left(\mathbb{C}^{n}, 0\right)$ e ainda

$$
\operatorname{dim}_{\mathbb{C}} Q\left(\left(F_{\mathbb{C}}\right)_{t}\right)=\text { constante, }
$$

pois $\operatorname{dim}_{\mathbb{C}} Q\left(\left(F_{\mathfrak{C}}\right)_{t}\right)=\operatorname{dim}_{\mathbb{R}} Q\left(F_{t}\right)$ (ver [2]). Mas, nos complexos, a dimensão da álgebra local coincide com a definição do grau de $\left(F_{\mathbb{C}}\right)_{t}$.

Afirmaçăo 1. Existe uma vizinhança $V$ de $0 \mathrm{em} \mathbb{C}^{n}$ tal que $\left(F_{\mathbb{C}}\right)_{t}^{-1}(0) \cap V=\{0\}$.

De fatto, suponha que não. Ou seja, suporha que exista uma sequência $\left(x_{n}, t_{n}\right) \in \mathbb{C}^{n} \times \mathbb{C}$ convergindo para $(0,0)$, tal que $\left(F_{\widetilde{C}}\right)_{t_{n}}\left(x_{n}\right)=0$. Daí, pelo Lema de Seleção da Curva, existe una curva $\gamma(s)=(x(s), t(s))$ passando por estes pontos, tal que $\gamma(0)=(0,0) \mathrm{c}$ $\left(F_{\mathbb{C}}\right)_{\ell(s)}(x(s))=0$. Mas, isso contraria a semi-continuidade superior e a constância da $\operatorname{dim}_{\mathbb{C}} Q\left(\left(F_{\mathbb{C}}\right)_{t}\right)$. Assim a Afirmação 1 está demonstrada.

Agora, considere a vizinhança $V_{0}=V \cap\left(\mathbb{R}^{n} \times\{0\}\right)$ em $\left(\mathbb{R}^{n}, 0\right)$.

Afirmação 2. $F_{t}^{-1}(0) \cap V_{0}=\{0\}$.

De fato, pois caso contrário, pelo mesmo argumento anterior, existiria uma curva em $\left(\mathbb{R}^{n}, 0\right)$ (que também é uma curva em $\left(\mathbb{C}^{n}, 0\right)$ ) e isto geraria um absurdo, conforme já vimos. Portanto, a Afirmação 2 está demonstrada.

Deste modo, como existe uma vizinhança $V_{0}$ de 0 em $\mathbb{R}^{n}$ tal que $F_{t}^{-1}(0) \cap V_{0}=\{0\}$, temos que o grau $\left(F_{t}\right)$ é constante. Pelo Teorema 2.17, $F_{t}$ e $F_{t^{\prime}}$ são $\mathcal{C}^{(0}$-K-equivalentes, quaisquer que sejam $t, t^{\prime} \in[0,1]$. 
De um modo geral, citamos no início deste capítulo que a $\mathcal{C}^{0}-\mathcal{K}$-deterrninação finita não é necessariamente uma propriedade aberta. Entretanto, no caso particular de germes analíticos tais que a dimensão da fonte é a mesma que a da meta $(n=p)$, temos o seguinte resultado:

Proposição 2.21. Sejam $f:\left(\mathbb{R}^{n}, 0\right) \rightarrow\left(\mathbb{R}^{n}, 0\right)$ um germe analítico $\mathcal{C}^{0}-\mathcal{K}$-finito e $F^{\top}:\left(\mathbb{R}^{n} \times \mathbb{R}, 0\right) \rightarrow\left(\mathbb{R}^{p}, 0\right)$ uma deformação analítica de $\int$. Pondo $F_{t}(x)=F(x, t)$ então para pequenos valores de $t, F_{t} e^{0} \mathcal{C}^{0} \mathcal{K}$-finito

Prova. Seja $A=\left\{(x, t) \in \mathbb{R}^{n} \times \mathbb{R} \mid x \in \Sigma F_{t}\right\}$.

Seja $\pi: A \cap F^{-1}(0) \rightarrow \mathbb{R}$ a projeção no segundo fator. Então $A \cap F^{-1}(0)$ é uma variedade analítica e $(0,0) \in \pi^{-1}(0)$ é um ponto isolado por hipótese. Assim, $\pi$ é uma aplicação finita na vizinhança de $(0,0)$ e o resultado segue.

\subsection{Outras propriedades da $\mathcal{C}^{0}-\mathcal{K}$-equivalência}

Nas seções anteriores, citamos os principais resultados conhecidos na literatura, sobre a $\mathcal{C}^{0}-\mathcal{K}$-equivalência. Nesta seção, apresentamos novos resultados e destacamos algurnas propriedades que diferenciam a $\mathcal{C}^{0}-\mathcal{K}$-equivalência da $\mathcal{K}$-equivalência e também da $\mathcal{C}^{0}$ - $\mathcal{A}$ equivalência.

O primeiro resultado, Proposição 2.22 , utiliza o conceito de função controle. Estas funções aparecerão também no Capítulo 5.

Una função controle $\rho: \mathbb{R}^{n} \rightarrow \mathbb{R}$ é uma função não-negativa tal que

i) $\rho(0) \neq 0$ ou

ii) $\rho(0)=0$ e $\rho$ satisfaz uma condição de Lojasiewcz, ou seja, existem constantes $c>0$ e $\alpha>0$ tais que $\rho(x) \geq c|x|^{\alpha}$.

Por exemplo, a função

$$
\rho(x)=x_{1}^{2}+\ldots+x_{n}^{2}
$$

é uma função controle, onde $x=\left(x_{1}, \ldots, x_{n}\right) \in \mathbb{R}^{n}$.

Proposição 2.22. Seja $f:\left(\mathbb{R}^{n}, 0\right) \rightarrow\left(\mathbb{R}^{p}, 0\right)$ e para cada $i=1, \ldots$, p considere funções controle $\rho_{i}: \mathbb{R}^{n} \rightarrow \mathbb{R}$, tais que $\rho=\left(\rho_{1}, \ldots, \rho_{p}\right): \mathbb{R}^{n} \rightarrow \mathbb{R}^{p}$. Então, $f$ e $f \cdot \rho=$ $\left(f_{1} \rho_{1}, \ldots, f_{p} \rho_{p}\right)$ são $\mathcal{C}^{0}-\mathcal{K}$-equivalentes. 
Prova. Observe que $f^{-1}(0)=(f \cdot \rho)^{-1}(0)$. Considere a homotopia

$$
F(x, t)=(1-t) f(x)+t(f \cdot \rho)(x), \quad x \in \mathbb{R}^{n}, \quad t \in[0,1] .
$$

Ou seja, para cada $i=1, \ldots, p$

$$
F_{i}(x, t)=(1-t) f_{i}(x)+t\left(f_{i} \rho_{i}\right)(x)=\left(1-t+t \rho_{i}(x)\right) f_{i}(x,) .
$$

Assim, $F_{t}:\left(\mathbb{R}^{n}, 0\right) \rightarrow\left(\mathbb{R}^{p}, 0\right)$ satisfaz as três condições do Lema 2.14. Logo, $f$ e $f \cdot \rho$ são $\mathcal{C}^{0}$ - $\mathcal{K}$-equivalentes.

Proposição 2.23. Seja $f=\left(f_{1}, \ldots, f_{p}\right):\left(\mathbb{R}^{n}, 0\right) \rightarrow\left(\mathbb{R}^{p}, 0\right)$ um germe analítico $\mathcal{C}^{0}-\mathcal{K}$ finito. Então, para um conjunto aberto e denso de parâmetros $\alpha=\left(\alpha_{1}, \ldots, \alpha_{p}\right) \in \mathbb{R}^{p}$, o germe de função

$$
f_{\alpha}(x)=\alpha_{1} f_{1}(x)+\ldots+\alpha_{p} f_{p}(x)
$$

$e^{0}-\mathcal{K}$-finito.

Prova. Considere a seguinte função:

$$
\begin{aligned}
H:\left(\mathbb{R}^{n}-\{0\}\right) \times\left(\mathbb{R}^{p}-\{0\}\right) & \rightarrow \mathbb{R} \\
\left(x, \alpha_{1}, \ldots, \alpha_{p}\right) & \mapsto \sum_{i=1}^{p} \alpha_{i} f_{i}(x) .
\end{aligned}
$$

Afirmamos que 0 é valor regular de $H$. De fato, suponha que 0 scja um valor crítico de $H$. Seja $q=\left(x_{0}, \alpha_{1}^{o}, \ldots, \alpha_{p}^{o}\right) \in\left(\mathbb{R}^{n}-\{0\}\right) \times\left(\mathbb{R}^{p}-\{0\}\right)$ um ponto crítico de $H$ tal que $H(q)=0$. Ent,ão,

$$
\left(\begin{array}{llll}
\frac{\partial H}{\partial x}(q) & \frac{\partial H}{\partial \alpha_{1}}(q) & \ldots & \frac{\partial H}{\partial \alpha_{p}}(q)
\end{array}\right)=\left(\begin{array}{llll}
0 & 0 & \ldots & 0
\end{array}\right)
$$

ou seja,

$$
\left(\begin{array}{llll}
\sum_{i=1}^{p} \alpha_{i}^{o} \operatorname{grad} f_{i}\left(x_{0}\right) & f_{1}\left(x_{0}\right) & \ldots & f_{p}\left(x_{0}\right)
\end{array}\right)=\left(\begin{array}{llll}
0 & 0 & \ldots & 0
\end{array}\right) .
$$

Então, $x_{0} \in f^{-1}(0)$ e os vetores $\operatorname{grad} f_{i}\left(x_{0}\right), i=1, \ldots, p$, são linearmente dependentes pois os $\alpha_{i}^{o}$ não são todos nulos. Mas isto é um absurdo pois nos pontos $x \in \mathbb{R}^{n}$ tais que $x \in f^{-1}(0)$, devemos ter os vetores grad $f_{i}(x), i=1, \ldots, p$, linearmente indepondentes, já que por hipótese $f^{-1}(0) \cap \Sigma f=\{0\}$.

Portanto 0 é valor regular de $H$. Pelo Teorema 1.36 (Lema de Transversalidade de Thom) temos que a aplicação

$$
F=H_{\mid\left(\alpha_{1}, \ldots, \alpha_{p}\right)}: \mathbb{R}^{n}-\{0\} \rightarrow \mathbb{R}
$$


tem 0 como valor regular para quase todo $\alpha_{1}, \ldots, \alpha_{p}$. Daí, $F^{-1}(0) \cap \Sigma F=\emptyset$.

Agora, se incluímos o ponto 0 na definição de $F$, obtemos a função $f_{\alpha}$ e temos que 0 é o único ponto singular de $f_{\alpha}$. Portanto, $f_{\alpha}$ é $\mathcal{C}^{0}-\mathcal{K}$-finito, como queríamos.

Corolário 2.24. Todo germe $f=\left(f_{1}, \ldots, f_{p}\right):\left(\mathbb{R}^{n}, 0\right) \rightarrow\left(\mathbb{R}^{p}, 0\right) \mathcal{C}^{0}-\mathcal{K}$-finitamente delerminado é $\mathcal{C}^{\prime \prime}-\mathcal{K}$-equivalente a u'm germe $g=\left(g_{1}, \ldots, g_{p}\right)$ tal que as funções coordenadas $g_{i}:\left(\mathbb{R}^{n}, 0\right) \rightarrow(\mathbb{R}, 0)$ são $\mathcal{C}^{0}-\mathcal{K}$-finitas.

Prova. Pela Proposição 2.23, existe um conjunto aberto e denso de parâmetros $\alpha \in \mathbb{R}^{p}$ tais que $f_{\alpha x} e^{0}-\mathcal{K}$-finito. Escolha $p$ vetores linearmente independentes

$$
\alpha^{1}=\left(\alpha_{1}^{1}, \ldots, \alpha_{p}^{1}\right), \ldots, \alpha^{p}=\left(\alpha_{1}^{p}, \ldots, \alpha_{p}^{p}\right)
$$

formando uma base para $\mathbb{R}^{p}$.

Defina $g=\left(g_{1}, \ldots, g_{p}\right):\left(\mathbb{R}^{n}, 0\right) \rightarrow\left(\mathbb{R}^{p}, 0\right)$ do seguinte modo:

$$
g_{i}=\alpha_{1}^{i} f_{1}+\ldots+\alpha_{p}^{i} f_{p}
$$

Pela Proposiçãa 2.23, $g_{i}$ i $\mathcal{C}^{0}-\mathcal{K}$-finito, para todo $i=1, \ldots, p$. Além disso, pela construção de $g$, temos que $f$ e $g$ são $\mathcal{C}^{0}-\mathcal{K}$-equivalentes.

Considere o subconjunto

$$
W=\left\{\alpha \in \mathbb{R}^{p} \mid f_{\alpha}:\left(\mathbb{R}^{n}, 0\right) \rightarrow(\mathbb{R}, 0) \text { não é } \mathcal{C}^{0}-\mathcal{K} \text {-finito }\right\} \subset \mathbb{R}^{p}
$$

onde $f, \alpha$ e $f_{\alpha}$ são como na Proposição 2.23. No complementar de $W$, estão os valores $\alpha \in \mathbb{R}^{p}$ tais que $f_{\alpha} \dot{e} \mathcal{C}^{0}-\mathcal{K}$-finito. Com isso, conseguimos particionar o conjunto dos germes $\mathcal{C}^{0}-\mathcal{K}$-finitos em um número finito de classes, com a propriedade que em cada classe todos os clementos são $\mathcal{C}^{0}-\mathcal{K}$-equivalentes (ver [44]). Cada uma destas classes é representada por um germe chamado elemento genérico. Ao contrário do que ocorre com a $\mathcal{K}$-equivalência, no caso topológico o fato dos germes serem $\mathcal{C}^{0}-\mathcal{K}$-equivalentes não implica que os elementos genéricos associados a eles sejam também $\mathcal{C}^{0}-\mathcal{K}$-equivalentes. De fato, considere os germes

$$
f(x, y)=\left(x^{2}-y^{2}, x^{4}+y^{4}\right) \text { e } g(x, y)=\left(x^{4}-y^{4}, x^{2}+y^{2}\right)
$$

De acordo com o Teorema 2.17 , eles são $\mathcal{C}^{0}-\mathcal{K}$-equivalentes pois ambos têm o mesmo grau. Podemos associar aos germes $f$ e $g$ os seguintes elementos genéricos

$$
f_{\alpha}(x, y)=\alpha_{1}\left(x^{2}-y^{2}\right)+\alpha_{2}\left(x^{4}+y^{4}\right), \quad \alpha_{1} \neq 0 \Rightarrow f_{\alpha} \stackrel{\mathcal{C}^{0}-\mathcal{K}}{\sim} x^{2}-y^{2}
$$




$$
g_{\beta}(x, y)=\beta_{1}\left(x^{4}-y^{4}\right)+\beta_{2}\left(x^{2}+y^{2}\right), \quad \beta_{2} \neq 0 \Rightarrow g_{\beta} \stackrel{\mathcal{C}^{0} \mathcal{K}}{\sim} x^{2}+y^{2} .
$$

Observe que $f_{\alpha}$ e $g_{\beta}$ não são $\mathcal{C}^{0}$ - $\mathcal{K}$-equivalentes. No entanto, se multiplicarmos as aplicaçốs $f$ e $g$ por convenientes controles, podemos obter elementos genéricos associados às novas aplicações, que são $\mathcal{C}^{0}-\mathcal{K}$-equivalentes. Observe que no caso dos germes $f$ e $g$ citados acima, se consideramos os controles $\rho=\left(\rho_{1}, \rho_{2}\right)$, com $\rho_{1}(x, y)=x^{2}+y^{2}$ e $\rho_{2}(x, y)=1$; e $\bar{\rho}=\left(\bar{\rho}_{1}, \bar{\rho}_{2}\right)$, com $\bar{\rho}_{1}(x, y)=1$ e $\bar{\rho}_{2}(x, y)=x^{2}+y^{2}$, segue pela Proposição 2.23 que,

$$
f=\left(f_{1}, f_{2}\right) \stackrel{\mathcal{C}^{0}-\mathcal{K}}{\sim}\left(f_{1} \rho_{1}, f_{2} \rho_{2}\right)=f \cdot \rho=\tilde{f}
$$

$$
g=\left(g_{1}, g_{2}\right) \stackrel{\mathcal{C}^{0} \mathcal{X}}{\sim}\left(g_{1} \bar{\rho}_{1}, g_{2} \bar{\rho}_{2}\right)=g \cdot \bar{\rho}=\tilde{g} .
$$

Os germes

$$
\tilde{f}(x, y)=\left(\left(x^{2}-y^{2}\right)\left(x^{2}+y^{2}\right), x^{4}+y^{4}\right) \text { e } \tilde{g}(x, y)=\left(x^{4}-y^{4},\left(x^{2}+y^{2}\right)^{2}\right)
$$

são agora $\mathcal{C}^{0}-\mathcal{K}$-equivalentes. O mesmo acontece com $\tilde{f}_{\alpha}$ e $\tilde{f}_{\beta}$, isto é, $\tilde{f}_{\alpha} \stackrel{\mathcal{L}^{0}-\mathcal{K}}{\sim} \tilde{f}_{\beta}$. Mais ainda, existe uma família

$$
F(x, t)=(1-t) \tilde{f}(x)+t \tilde{g}(x)
$$

tal que para qualquer $t \in[0,1]$,

$$
\tilde{f} \stackrel{C^{0}-\mathcal{K}}{\sim} F_{t} \stackrel{C^{0}-\mathcal{K}}{\sim} \tilde{g}
$$

Esta análise permite concluir que se for possível multiplicar germes de aplicaçôes por convenientes controles, podemos restabelecer para a $\mathcal{C}^{0}-\mathcal{K}$-equivalência o mesmo tipo do propriedade preservada pelo grupo $\mathcal{K}$, para os elementos genéricos. Este tipo de argumento pode ser um caminho na tentativa de obter resultados topológicos, análogos ao Corolário 1.46. Mas, por enquanto, ainda não obtivemos resultados nesta linha.

Finalizamos esta seção observando que no caso dos germes de aplicações polinomiais $\left(\mathbb{R}^{n}, 0\right) \longrightarrow\left(\mathbb{R}^{p}, 0\right)$ de grau $\leq k, \mathcal{C}^{0}$ - $\mathcal{K}$-finitos, Nishimura [44] provou que o número das $\mathcal{C}^{0}-\mathcal{K}$-órbitas é finito. Seu argumento utiliza a caracterização geométrica da $\mathcal{C}^{0}-\mathcal{K}$ determinação finita (Proposição 2.6) e a versão local do segundo lema de isotopia de Thom (Teorema 1.19). $\Lambda$ finitude das $\mathcal{C}^{0}-\mathcal{K}$-órbitas deixa mais tratável o estudo da $\mathcal{C}^{0}-\mathcal{K}$ classificação dos germes de aplicações polinomiais.

Vale ressaltar que no caso da $\mathcal{C}^{0}-\mathcal{A}$-equivalência (ou equivalência topológica), I. Nakai [42] apresenta exemplos de famílias de germes de aplicações polinomiais de $\mathbb{R}^{n}$ em $\mathbb{R}^{p}$ de grau $k$ com $n, p, k \geq 3$ ou $n \geq 3, p \geq 2 \mathrm{e} k \geq 4$, as quais tôm infinitas $\mathcal{C}^{0}$ - $\mathcal{A}$-órbitas. Mesmo 
restringindo a análise aos germes $\mathcal{C}^{0}-\mathcal{K}$-finitos, observamos que o número de $\mathcal{C}^{0}$ - $\mathcal{A}$-órbitas continua infinito (ver Nishimura [44]).

\subsection{Interpretação geométrica}

J. Montaldi ([39]) estabeleceu uma interpretação geométrica natural para a $\mathcal{K}$-equivalência de Mather, através do contato entre subvariedades. Como já vimos no Capítulo 1 , Teorema 1.51 , dois pares de subvariedades do $\mathbb{R}^{n},\left(\mathcal{X}_{1}, \mathcal{Y}_{1}\right)$ e $\left(\mathcal{X}_{2}, \mathcal{Y}_{2}\right)$, têm o mesmo tipo de contato na origem se, $\mathrm{c}$ somente se, as aplicações $f_{1} \circ g_{1}$ e $f_{2} \circ g_{2}$ são $\mathcal{K}$-equivalentes, onde $g_{i}:\left(\mathcal{X}_{i}, 0\right) \rightarrow\left(\mathbb{R}^{n}, 0\right)$ são germes de imersões e $f_{i}:\left(\mathbb{R}^{n}, 0\right) \rightarrow\left(\mathbb{R}^{p}, 0\right)$ germes de submersões, com $\mathcal{Y}_{i}=f_{i}^{-1}(0), i=1,2$. Em particular, dois germes de aplicaçõos $f, g:\left(\mathbb{R}^{n}, 0\right) \rightarrow\left(\mathbb{R}^{p}, 0\right)$ são $\mathcal{K}$-equivalentes se, e somente se, seus gráficos têm o mesmo contato com o $\mathbb{R}^{n}$ na origem.

Agora, nosso objetivo é tentar estabelecer resultados análogos aos citados acima, para a $\mathcal{C}^{0}$-K-equivalência. Para isto, introduzimos o conceito de mesmo tipo de contato topológico.

Sejam $\mathcal{X}_{i}$ e $\mathcal{Y}_{i}$, subvariedades do $\mathbb{R}^{n}$, com $\operatorname{dim} \mathcal{X}_{1}=\operatorname{dim} \mathcal{X}_{2}$ c dim $\mathcal{Y}_{1}=\operatorname{dim} \mathcal{Y}_{2}$, $i=1,2$.

Definição 2.25. Dizemos que o par $\left(\mathcal{X}_{1}, \mathcal{Y}_{1}\right)$ tem o mesmo tipo do contato topológico que o par $\left(\mathcal{X}_{2}, \mathcal{Y}_{2}\right)$ na origen se existe um germe de homeomorfismo $H:\left(\mathbb{R}^{n}, 0\right) \longrightarrow\left(\mathbb{R}^{n}, 0\right)$ tal que $H\left(\mathcal{X}_{1}\right)=\mathcal{X}_{2}$ e $H\left(\mathcal{Y}_{1}\right)=\mathcal{Y}_{2}$.

Notação: $\mathcal{C}^{0}-K\left(\mathcal{X}_{1}, \mathcal{Y}_{1}, 0\right)=\mathcal{C}^{0}-K\left(\mathcal{X}_{2}, \mathcal{Y}_{2}, 0\right)$.

Assim, considerando $\mathcal{X}_{i}, \mathcal{Y}_{i}, g_{i}$ e $f_{i}$ como citados acima, estamos interessados em obter uma versão topológica do Teorema 1.51. Ou seja, gostaríamos de recscrever o Teorema 1.51 do seguinte rnodo:

"Os pares de subvariedades $\left(\mathcal{X}_{1}, \mathcal{Y}_{1}\right)$ e $\left(\mathcal{X}_{2}, \mathcal{Y}_{2}\right)$ têm o mesmo tipo de contato topológico na origem $\Leftrightarrow$ as aplicações $f_{1} \circ g_{1}$ e $f_{2} \circ g_{2}$ são $\mathcal{C}^{0}$ - $\mathcal{K}$-equivalentes."

Observe que a implicação $(\Leftarrow)$ é sempre verdadeira. No entanto, não conseguimos ainda mostrar que o resultado acima vale em geral. Mostramos que é verdade para o caso de hipersuperfícies, conforme veremos na última parte do Capítulo 4. Para o caso geral, se tentamos imitar a prova de Montaldi para o caso diferenciável, esbarramos na falta de uma versão topológica do Lema de Hadamard (ver [23], [39]) para provar a implicação $(\Rightarrow)$. Ainda no Capítulo 4 , mostramos que tanto para a categoria dos germes de funções 
definívcis, quanto para a categoria dos germes de funçñes $\mathcal{C}^{0}-\mathcal{K}$-finitas, os germes são $\mathcal{C}^{0}-\mathcal{K}$ equivalentes se, e somente se, seus gráficos têm o mesmo tipo de contato topológico com o $\mathbb{R}^{n}$ na origem. No Capítulo 3, para germes de aplicações $\left(\mathbb{R}^{n}, 0\right) \rightarrow\left(\mathbb{R}^{p}, 0\right)$ com $n<p$, obtemos trivialmente este resultado como consequência do Teorema 3.6 . 


\title{
Capítulo 3
}

\section{$\mathcal{C}^{0}$ - $\mathcal{K}$-equivalência de aplicações}

\author{
Caso $n<p$
}

Neste capítulo analisamos o que ocorre com a $\mathcal{C}^{0}$ - $\mathcal{K}$-equivalência para germes de aplicações $f:\left(\mathbb{R}^{n}, 0\right) \rightarrow\left(\mathbb{R}^{p}, 0\right), n<p$. Conforme veremos no Teorema 3.6, quando $n<p$ a $\mathcal{C}^{0}-\mathcal{K}-$ equivalência não distingue germes $\mathcal{C}^{0}-\mathcal{K}$-finitos. Os excmplos a seguir ilustram o motivam este fato.

Exemplo 3.1. Os germes $f(x)=\left(x^{2}, 0\right)$ e $g(x)=(x, 0)$ são $\mathcal{C}^{0}-\mathcal{K}$-equivalentes.

De fato, observe inicialmente que os germes $f(x)=\left(x^{2}, 0\right)$ e $\psi_{1}(x)=\left(x^{2}, x^{3}\right)$ são $\mathcal{K}$-equivalentes (Proposição 1.45). O mesmo acontece com os germes $g(x)=(x, 0)$ e $\psi_{2}(x)=\left(x^{2}, x\right)$.

Além disso, $\psi_{2}(x)=\left(x^{2}, x\right)$ e $\psi_{1}(x)=\left(x^{2}, x^{3}\right)$ são $\mathcal{C}^{0}$-K -equivalentes pois o par de homeomorfismos $h(x)=x$ e $H\left(x, y_{1}, y_{2}\right)=\left(x, y_{1}, y_{2}^{3}\right)$ satisfaz as hipóteses da Definição 2.1. Assim,

$$
f \stackrel{\mathcal{K}}{\sim} \psi_{1} \stackrel{\mathcal{C}^{0} \mathcal{K}}{\sim} \psi_{2} \stackrel{\mathcal{K}}{\sim} g
$$

e, portanto, $f$ e g são $\mathcal{C}^{0}-\mathcal{K}$-equivalenles.

No entanto, observe que $x$ e $x^{2}$ não são $\mathcal{C}^{0}-\mathcal{K}$-equivalentes como germes de funções reais em uma variável.

Exemplo 3.2. Os germes $f(x)=\left(x^{2}, x^{3}\right)$ e $g(x)=\left(x^{4}, x^{5}\right)$ são $\mathcal{C}^{0}-\mathcal{K}$-equivalentes. De fato, considerando as funções controle $\rho_{1}(x)=\rho_{2}(x)=x^{2}$, seyue pela Proposição 2.22 que os germes $f=\left(f_{1}, f_{2}\right)$ e $f \cdot \rho=\left(f_{1} \rho_{1}, f_{2} \rho_{2}\right)=g$ são $\mathcal{C}^{0}-\mathcal{K}$-equivalentes. Este mesmo argumento prova que os germes $f(x)=\left(x^{2}, 0\right)$ e $g(x)=\left(x^{4}, 0\right)$ são também $\mathcal{C}^{0}-\mathcal{K}$-equivalentes.

Observação. Mais geralmente, seguindo os argumentos dos exemplos acima, não é 
difícil mostrar que $f(x)=\left(x^{l}, 0\right)$ é $\mathcal{C}^{0}-\mathcal{K}$-equivalente a $g(x)=\left(x^{s}, 0\right)$, quaisquer que sejam $l, s$ inteiros positivos.

Segue da teoria de determinação finita $|65|$ que sc $f:(\mathbb{R}, 0) \rightarrow\left(\mathbb{R}^{2}, 0\right)$ é um germe $\mathcal{K}$-finitamente determinado, então podemos supor $f \stackrel{\mathcal{C}^{0}-\mathcal{K}}{\sim}\left(t^{k}, 0\right)$, para algum $k$ inteiro pasitivo. Então temos o seguinte resultado:

Proposição 3.3. Sejam $f, g:(\mathbb{R}, 0) \rightarrow\left(\mathbb{R}^{2}, 0\right)$ germes $\mathcal{K}$-finitamente determinados. Ent tão $f$ eg são $\mathcal{C}^{0}-\mathcal{K}$-equivalentes.

Prova. Da hipótese que $f$ e $g$ são $\mathcal{K}$-finitamente determinados, segue que $f \stackrel{\mathcal{L}^{0}-\mathcal{K}}{\sim}\left(t^{k}, 0\right)$ e $g \stackrel{C^{0}-\mathcal{K}}{\sim}\left(t^{l}, 0\right)$, com $k$ e $l$ inteiros positivos. Então, pela Observação acima, $f$ e $g$ são $\mathcal{C}^{0}-\mathcal{K}$ equivalentes.

Note que a Proposição 3.3 pode ser generalizada para germes $(\mathbb{R}, 0) \rightarrow\left(\mathbb{R}^{p}, 0\right)$, de forma natural.

A seguir ilustraremos um exemplo de germes $\left(\mathbb{R}^{2}, 0\right) \rightarrow\left(\mathbb{R}^{3}, 0\right), \mathcal{C}^{0}-\mathcal{K}$-finitos, que são $\mathcal{C}^{0}-\mathcal{K}$-equivalentes.

Exemplo 3.4. Os germes $f(x, y)=(x, y, 0)$ e o cross-cap $g(x, y)=\left(x, y^{2}, x y\right)$ (ver [381) são $\mathcal{C}^{0}-\mathcal{K}$-equivalentes.

De fato, observe primeiramente que $f \stackrel{\mathcal{K}}{\sim}\left(x, y^{2}, y\right)$ e $g \stackrel{\mathcal{X}}{\sim}\left(x, y^{2}, y^{3}\right)$. Basta mostrar que $\left(x, y^{2}, y\right) \stackrel{\mathcal{C}^{0} \mathcal{K}}{\sim}\left(x, y^{2}, y^{3}\right)$.

Para isto, considere os homeomorfismos

$$
h(x, y)=\left(x^{3}, y^{3}\right) \text { e } H\left(x, y, z_{1}, z_{2}, z_{3}\right)=\left(x^{3}, y^{3}, z_{1}^{3}, z_{2}^{3}, z_{3}^{9}\right)
$$

Eles estabelecem a $\mathcal{C}^{0}$-Ke-equivalência entre os germes $\left(x, y^{2}, y\right)$ e $\left(x, y^{2}, y^{3}\right)$. Assim,

$$
f \stackrel{\mathcal{K}}{\sim}\left(x, y^{2}, y\right) \stackrel{\mathcal{C}^{0} \mathcal{K}}{\sim}\left(x, y^{2}, y^{3}\right) \stackrel{\mathcal{K}}{\sim} g
$$

e, portanto, $f$ e g são $\mathcal{C}^{0}-\mathcal{K}$-equivalentes.

De forma geral, dado um germe $f:\left(\mathbb{R}^{2}, 0\right) \rightarrow\left(\mathbb{R}^{3}, 0\right)$ de coposto 1 , podemos supor

$$
f \stackrel{\mathcal{C}^{0} \mathcal{K}}{\sim}\left(x, f_{1}(y), f_{2}(y)\right)
$$

Mas, $\left(x, f_{1}(y), f_{2}(y)\right) \stackrel{\mathcal{C}^{0} \mathcal{K}}{\sim}\left(x, a y^{k}+\ldots, b y^{l}+\ldots\right), k$ e $l$ inteiros positivos.

Se, $a \neq 0$ e $k \leq l$ obtemos $f \stackrel{\mathcal{C}^{0}-\mathcal{K}}{\sim}\left(x, y^{k}, 0\right)$.

Pergunta: $\quad\left(x, y^{k}, 0\right) \stackrel{\mathcal{C}^{0}-\mathcal{K}}{\sim}\left(x, y^{l}, 0\right)$ ? 
A resposta é sim e faremos um esboço da prova para o caso $k<l$, com $k$ par e $l$ ímpar. Os outros casos são análogos.

Primeiramente, observe que:

$$
\left(x, y^{l}, 0\right) \stackrel{\mathfrak{C}^{0}-\mathcal{K}}{\sim}(x, y, 0)
$$

devido o homeomorfismo natural $H\left(x, y, z_{1}, z_{2}, z_{3}\right)=\left(x, y, z_{1}, z_{2}^{l}, z_{3}\right)$.

Agora,

$$
\left(x, y^{k}, 0\right) \stackrel{\mathcal{K}}{\sim}\left(x, y^{k}, y^{k+1}\right) \stackrel{\mathcal{C}^{0} \mathcal{K}}{\sim}\left(x, y^{k}, y\right) \stackrel{\mathcal{K}}{\sim}(x, y, 0) .
$$

Portanto, $\left(x, y^{k}, 0\right) \stackrel{\mathcal{C}^{0}-\mathcal{K}}{\sim}\left(x, y^{l}, 0\right)$, como queríamos.

De um modo geral, no caso $n<p$, ć possível mostrar que quaisquer dois germes $\mathcal{C}^{0}$-K $\mathcal{K}$-finitamente determinados são $\mathcal{C}^{0}-\mathcal{K}$-equivalentes. A confirmação disto é dada pelo Teorema 3.6. Este teorema decorre do Lema 2.14 e do Teorema do Cone de Fukuda (ver [19]).

Teorema 3.5. (Teorema do Cone de Fukuda (19|) Suponha $n \leq p$. Então, dado um subconjunto semialgébrico $W$ de $J^{r}(n, p)$, existe um inteiro $s$, dependendo apenas de $n, p$ e r, e um subconjunto semialgébrico fechado $\Sigma_{W}$ de $\left(\pi_{r}^{s}\right)^{-1}(W)$ com codimensão $\geq 1$ tais que para qualquer aplicação $\mathcal{C}^{\infty} \quad \int: \mathbb{R}^{n} \rightarrow \mathbb{R}^{p}$ com $j^{s} f(0) \in\left(\pi_{r}^{s}\right)^{-1}(W)-\Sigma_{W}$, existe um número positivo $\varepsilon_{0}$ tal que para qualquer número $\varepsilon$ com $0<\varepsilon \leq \varepsilon_{0}$ temos

i) se $n \neq 4,5, f^{-1}\left(S_{\varepsilon}^{p-1}\right)$ é uma variedade sem fronteira homeomorfa a $(n-1)$-esfera unitária padrão $S^{n-1}$;

ii) a restrição $f: f^{-1}\left(D_{\varepsilon}^{p}\right) \rightarrow D_{\varepsilon}^{p} e ́ \mathcal{C}^{0}-\mathcal{A}$-equivalente ao cone

$$
c(f): f^{-1}\left(S_{\varepsilon}^{p-1}\right) \times[0, \varepsilon) / f^{-1}\left(S_{\varepsilon}^{p-1}\right) \times\{0\} \rightarrow S_{\varepsilon}^{p-1} \times[0, \varepsilon) / S_{\varepsilon}^{p-1} \times\{0\},
$$

onde $c(f)=(f(x), t)$.

Um germe f para o qual existe $\varepsilon_{0}$ satisfazendo i) e ii) é chamado tipo cone.

Teorema 3.6. Sejam $f, g:\left(\mathbb{R}^{n}, 0\right) \longrightarrow\left(\mathbb{R}^{p}, 0\right)$ germes de aplicações $\mathcal{C}^{0}$-K -finitamente determinados e $n<p, n \neq 4,5$. Então, $f$ e g são $\mathcal{C}^{0}-\mathcal{K}$-equivalentes.

Prova. Como $f$ (resp. $g$ ) é $\mathcal{C}^{0}-\mathcal{K}$-finitamente determinado, pelo Teorema 3.5 , existe um germe de aplicação do tipo cone $\tilde{f}:\left(\mathbb{R}^{n}, 0\right) \longrightarrow\left(\mathbb{R}^{p}, 0\right)$ tal que $f \dot{e} \mathcal{C}^{0}-\mathcal{K}$-equivalente a $\tilde{f}$ (resp. existe $g$ tal que $g$ é $\mathcal{C}^{0}-\mathcal{K}$-equivalente a $\tilde{g}$ ).

Segue do Teorema 3.5 i) que existe um homeomorfismo $\phi_{f}: S_{\varepsilon_{0}}^{n-1} \rightarrow \tilde{f}^{-1}\left(S_{\varepsilon_{0}}^{p-1}\right)$ (resp). $\left.\phi_{g}: S_{\varepsilon_{0}}^{n-1} \rightarrow \tilde{f}^{-1}\left(S_{\varepsilon_{0}}^{p-1}\right)\right)$. Agora, defina a aplicação $F: S_{\varepsilon_{0}}^{n-1} \rightarrow S_{\varepsilon_{0}}^{p-1}$ por $F(x)=\tilde{f} \circ \phi_{f}(x)$ (resp. $G(x)=\tilde{g} \circ \phi_{g}$ ). 
Por hipótese $n<p$, então $F$ e $G$ são homotópicas, ou seja, existe uma homotopia $H_{t}: S_{\varepsilon_{0}}^{n-1]} \rightarrow S_{\varepsilon_{0}}^{p-1}, t \in[0,1]$ tal que $I I_{0}=F$ e $I I_{1}=G$.

Considere $c\left(H_{\ell}\right): S_{\varepsilon_{0}}^{n-1} \times[0,1] / S_{\varepsilon_{0}}^{n-1} \times\{0\} \rightarrow S_{\varepsilon_{0}}^{p-1} \times[0,1] / S_{\varepsilon_{0}}^{p-1} \times\{0\}$ o conc desta homotopia, ou seja, $c\left(H_{\ell}\right)(x, s)=\left(H_{t}(x), s\right)$.

Então, $c\left(H_{0}\right)=c(F), c\left(H_{1}\right)=c(G) \mathrm{e} c\left(I_{t}\right)^{-1}(0)=\{0\}$ para qualquer $t \in[0,1]$. Além disso, como $c\left(H_{t}\right)$ é o cone de $I I_{t}$, por compacidade de $[0,1]$ existe um subconjunto finito $\left\{t_{0}, \ldots, t_{k}\right\}$ de $[0,1]$ tal que $0=t_{0}<\ldots<t_{k}=1 \mathrm{e}$, para qualquer inteiro $i$, com $0 \leq i \leq k-1$, c qualquer $t \in\left[t_{i}, t_{i+1}\right]$, o vetor $c\left(H_{t}\right)(x) \notin\left\{\alpha c\left(H_{t_{i}}\right) \mid \alpha \in \mathbb{R}_{-}\right\}$para qualquer $x \in S_{\varepsilon_{\mathcal{U}}}^{n-1} \times[0,1] / S_{\varepsilon_{\mathfrak{U}}}^{n-1} \times\{0\} \approx D_{\varepsilon}^{n-1}$.

Pelo Lema 2.14, $c(F)$ e $c(G)$ são $\mathcal{C}^{0}-\mathcal{K}$-equivalentes.

Portanto,

$$
f \stackrel{\mathcal{C}^{0}-\mathcal{K}}{\sim} \tilde{f} \tilde{C}^{\mathcal{C}^{0} \mathcal{A}} \sim(\tilde{f}) \stackrel{\mathcal{C}^{0}-\mathcal{R}}{\sim} c(F) \stackrel{\mathcal{C}^{0}-\mathcal{K}}{\sim} c(G) \stackrel{\mathcal{C}^{0} \sim \mathcal{R}}{\sim} c(\tilde{g}) \stackrel{\mathcal{C}^{0} \mathcal{A}}{\sim} \tilde{g} \stackrel{\mathcal{C}^{0}-\mathcal{K}}{\sim} g .
$$

Então, $f$ e $g$ são $\mathcal{C}^{0}-\mathcal{K}$-equivalentes.

Vale observar, que no caso de germes de aplicações $\mathcal{C}^{0}-\mathcal{K}$-finitos com $n<p$, segue do Teorema 3.6 que uma versão topológica dos resultados de Mather-Montaldi é automaticamente verdadeira. 


\section{Capítulo 4}

\section{$\mathcal{C}^{0}-\mathcal{K}$-equivalência de funções}

Este capítulo é dedicado à $\mathcal{C}^{0}$-K-equivalôncia dos germes de funções $\left(\mathbb{R}^{n}, 0\right) \rightarrow \mathbb{R}$. Os principais resultados apresentados aqui encontram-se em [1].

Para classificação de germes, um dos primeiros problemas que surgem é o da finitude das classes de equivalência.

T. Fukuda [18] provou que o conjunto dos germes de funções polinomiais $f:\left(\mathbb{R}^{n},(0) \longrightarrow \mathbb{R}\right.$ de grau limitado apresenta um número finito de tipos topológicos. Portanto, quando trabalhamos com funções analíticas finitamente determinadas ou, equivalentemente, com funções polinomiais, temos a finitude das classes.

Em [6]. Benedetti e Shiota provaram que o conjunto das classes de equivalência dos germes de funções polinomiais, com respeito à $\mathcal{A}$-equivalência topológica é finito. Este resultado foi generalizado por M. Coste [13] para funções definíveis em estruturas ominimais. Claramente, estes resultados são verdadeiros para a $\mathcal{K}$-equivalência topológica, já que a $\mathcal{A}$-equivalêncía topológica implica na $\mathcal{K}$-equivalência topológica. $\Lambda$ ssim, a questão da classificação destes germes pode ser perguntada e, portanto, podemos abordá-la neste capítulo.

Embora o problema da finitude esteja resolvido para germes de funções analiticas, enunciaremos a seguir um teorema de finitude na categoria dos conjuntos subanalíticos, a título de ilustração. Além disso, a prova que sugerimos é independente e utiliza uma adaptação do Lerna 2.14 para a $\mathcal{C}^{0}-\mathcal{K}$-equivalência subanalitica, isto é, basta substituirmos homeomorfismo por homeomorfismo subanalítico (isto é, o gráfico da aplicação é um conjunto subanalítico) na Definição 2.1.

Teorema 4.1. O conjunto das classes de equivalência dos germes de funçöes analíticas $f:\left(\mathbb{R}^{n}, 0\right) \longrightarrow \mathbb{R}$ módulo a $\mathcal{C}^{0}-\mathcal{K}$-equivalência subanalítica é enumerável. Além disso, para $n=2$, cada classe de $\mathcal{C}^{0}-\mathcal{K}$-equivalência subanalitica contém um representante polinomial. 
Prova. Scja $\left\{V_{1}, \ldots, V_{n}, \ldots\right\}$ o conjunto enumerável de classes de equivalência topológicas de germes de subconjuntos analíticos de $\left(\mathbb{R}^{n}, 0\right)$ (ver [29]). Para cada $V_{i}$, sejam $\left\{V_{i_{1}}, \ldots, V_{i_{k_{i}}}\right\}$ as componentes conexas de $\mathbb{R}^{n}-V_{i}$ e seja $\Sigma_{i}$ o conjunto formado por $2^{k_{i}}$ funçōes contínuas $\int: \mathbb{R}^{n}-V_{i} \longrightarrow\{-1,1\}$ e constantes em cada $V_{i_{j}}, \quad j=1, \ldots, k_{i}$.

Então, dada $g:\left(\mathbb{R}^{n}, 0\right) \longrightarrow \mathbb{R}$ analítica, existe $h:\left(\mathbb{R}^{n}, 0\right) \longrightarrow \mathbb{R}^{n}$ homeomorfismo subanalítico tal que $h\left(g^{-1}(0)\right)=V_{i}$ para algum $i$ (ver $\left.[40]\right)$.

Podemos assumir $g^{-1}(0)=V_{i}$ para algum $i$. Dessa forma, existe $f \in \Sigma_{i}$ tal que $\operatorname{sinal}(f)=\operatorname{sinal}(g)$ restrito a $\mathbb{R}^{n}-V_{i}$. Usando o Lema $2.14, f$ é $\mathcal{C}^{0}-\mathcal{K}$-subanaliticamente equivalente a $g$. Isto é possível pois os homeomorfismos construídos na prova do Lema 2.14 são subanalíticos. A segunda parte do teorema sairá como decorrência dos resultados deste capítulo, como veremos posteriormente no Teorema 4.5 .

No decorrer deste capítulo, apresentaremos na Seção 4.1 um invariante completo para germes de funções, chamado função tenda. A teoria é apresentada na linguagem das estruturas o-minimais. Usamos a versão definível do Lema 2.14 como principal ferramenta dos resultados da seção.

A Seção 4.2 é dedicada a um caso especial: os germes de funções analíticas. Para germes de função analítica o $\mathcal{K}$-invariante definido na Scção 4.1 é muito simples. $\mathrm{O}$ invariante é uma sequência finita de elementos iguais a 1 ou -1 . Provamos que todos estes invariantes, no caso $n=2$, admitem uma realização polinomial. Finalmente, para $n=2$, apresentamos uma forma normal para germes de funções $\mathcal{C}^{0}-\mathcal{K}$-finitamente determinados. Note que estes invariantes são criados para todas as singularidades definíveis e rãa apenas para o caso de singularidades genéricas, como na teoria clássica de singularidades.

\subsection{Funções tenda}

Seja $M \subset \mathbb{R}^{n}$ um politopo convexo $(n-1)$-dimensional. Seja $Z \subset M$ um subconjunto fechado tal que:

- $Z$ é uma união de faces de $M$;

- A codimensão de $Z$ em $M$ é diferente de zero.

Seja $\left\{U_{i}\right\}$ a família de componentes conexas de $M-Z$. A coleção $\left\{U_{i}\right\}$ é chamada uma $\mathcal{K}$-decomposição de $M$ e o conjunto $Z$ é chamado de lugar dos zeros de $\left\{U_{i}\right\}$.

Seja \|\|$_{M}$ uma norma em $\mathbb{R}^{n}$ tal que o politopo $M$ é realizado como uma esfera unitária. Seja 


$$
\tilde{Z}=\left\{x \in \mathbb{R}^{n}-\{0\} \mid \frac{x}{\|x\|_{M}} \in Z\right\} \text { e } \tilde{U}_{i}=\left\{x \in \mathbb{R}^{n}-\{0\} \mid \frac{x}{\|x\|_{M}} \in U_{i}\right\} .
$$

A coleção $\left\{\tilde{U}_{i}\right\}$ é chamada $\mathcal{K}$-decomposição de $\mathbb{R}^{n}$ associada a $\left\{U_{i}\right\}$ e o conjunto $\tilde{Z}$ é chamado lugar dos zeros de $\left\{\tilde{U}_{i}\right\}$.

Sejam $M_{1}, M_{2}$ politopos, $Z_{1}, Z_{2}$ o lugar dos zeros e $\left\{U_{i}^{1}\right\},\left\{U_{j}^{2}\right\}$ duas $\mathcal{K}$-decomposições. $\Lambda$ s $\mathcal{K}$-decomposições são chamadas combinatorialmente equivalentes se existem triangulações dos pares $\left(M, Z_{1}\right),\left(M, Z_{2}\right)$ e um isomorfismo simplicial entre estas triangulaçõess.

Uma função $T_{i}: \mathbb{R}^{n} \rightarrow \mathbb{R}$ dada por

$$
T_{i}(x)= \begin{cases}\operatorname{dist}\left(\mathrm{x}, \partial \tilde{\mathrm{U}}_{\mathrm{i}}\right) & \text { se } \mathrm{x} \in \tilde{\mathrm{U}}_{\mathrm{i}} \\ 0 & \text { caso contrário }\end{cases}
$$

é chamada função tenda elementar. As funções $\sum_{i} a_{i} T_{i}$, onde $a_{i}$ é igual a $-1,0$ on 1 , são chamadas funções tenda associadas à $\mathcal{K}$-decomposição $\left\{U_{i}\right\}$.

Sejam $\left\{\tilde{U}_{i}\right\}$ e $\left\{\tilde{V}_{j}\right\}$ duas $\mathcal{K}$-decomposições de $\mathbb{R}^{n}$. Sejam $\alpha, \beta$ funções tenda associadas $\grave{a} \tilde{U}_{i}$ e $\tilde{V}_{j}$ respectivamente. As funções $\alpha$ e $\beta$ são ditas combinatorialmente equivalentes se existe um germe de isomorfismo simplicial $h:\left(\mathbb{R}^{n}, 0\right) \rightarrow\left(\mathbb{R}^{n}, 0\right)$ tal que, para cada $\tilde{U}_{i}$, existe $\tilde{V}_{j}$ tal que $h\left(\tilde{U}_{i}\right)=\tilde{V}_{j}$ e sinal $[\alpha(x)]=\operatorname{sinal}[\beta(h(x))]$. Claramente, se duas funções tenda são combinatorialmente equivalentes então as $\mathcal{K}$-decomposições dos correspondentes politopos são combinatorialmente equivalentes.

Podemos adaptar a Definição 2.1 na linguagem do estruturas o-minimais do seguinte modo:

Definição 4.2. Seja $\mathcal{S}$ uma estrutura o-minimal sobre $\mathbb{R}$. Sejam $f, g:\left(\mathbb{R}^{n}, 0\right) \rightarrow \mathbb{R}$ germes de funcões contínuas definiveis em $\mathcal{S}$. Os germes $f$ e g são chamados topologicamente $\mathcal{K}$-equivalentes em $\mathcal{S}$ se existem germes de homeomorfismos definiveis em $\mathcal{S}$ $h:\left(\mathbb{R}^{n}, 0\right) \rightarrow\left(\mathbb{R}^{n}, 0\right)$ eH $:\left(\mathbb{R}^{n} \times \mathbb{R}, 0\right) \rightarrow\left(\mathbb{R}^{n} \times \mathbb{R}, 0\right)$ com a propriedade $I\left(\mathbb{R}^{n} \times\{0\}\right)=$ $\mathbb{R}^{n} \times\{0\}$ tais que os seguintes diagramas comutam:

$$
\begin{aligned}
& \left(\mathbb{R}^{n}, 0\right) \stackrel{(i d, f)}{\longrightarrow}\left(\mathbb{R}^{n} \times \mathbb{R}, 0\right) \stackrel{\pi_{n}}{\longrightarrow}\left(\mathbb{R}^{n}, 0\right) \\
& h \downarrow \quad H \downarrow \quad h \downarrow \\
& \left(\mathbb{R}^{n}, 0\right) \stackrel{(i d, g)}{\longrightarrow}\left(\mathbb{R}^{n} \times \mathbb{R}, 0\right) \stackrel{\pi_{n}}{\longrightarrow}\left(\mathbb{R}^{n}, 0\right)
\end{aligned}
$$

onde id: $\left(\mathbb{R}^{n}, 0\right) \rightarrow\left(\mathbb{R}^{n}, 0\right)$ ć a aplicação identidade e. $\pi_{n}:\left(\mathbb{R}^{n} \times \mathbb{R}, 0\right) \rightarrow\left(\mathbb{R}^{n}, 0\right)$ a projeção canônica dada por $\pi_{n}(x, t)=x,(x, t) \in \mathbb{R}^{n} \times \mathbb{R}$. 
Teorema 4.3. Seja $\mathcal{S}$ uma estrutura o-minimal sobre $\mathbb{R}$.

1. Seja $f:\left(\mathbb{R}^{n}, 0\right) \rightarrow \mathbb{R}$ um germe de funç̧ão contínua definível em $\mathcal{S}$. Então, existe uma $\mathcal{K}$-decomposição de $\mathbb{R}^{n}$ e uma função tenda $\alpha:\left(\mathbb{R}^{n}, 0\right) \rightarrow \mathbb{R}$ tal que $f$ e $\alpha$ são topologicamente $\mathcal{K}$-equivalentes em $\mathcal{S}$.

2. Duas funções tenda säo topologicamente $\mathcal{K}$-equivalentes $\mathrm{em} \mathcal{S}$ se, e somente se, elas são combinatorialmente equivalentes.

Lsamos a seguinte versão do Lema 2.14:

Lema 4.4. Seja $\mathcal{S}$ uma estrutura o-minimal sobre $\mathbb{R}$. Sejam $f, g: U \rightarrow \mathbb{R}$ funçóes continuas definiveis em $\mathcal{S}$, onde $U$ é uma vizinhança da origem em $\mathbb{R}^{n}$. Se $\frac{f(x)}{g(x)}>0$, para cada $x \in U-f^{-1}(0)$, então $f$ e g são topologicamente $\mathcal{K}$-equivalentes em $\mathcal{S}$.

Prova. Considere a família $F_{t}(x)=(1-t) f(x)+t g(x), t \in[0,1]$. Das hipóteses do lema segue que $F_{t}^{-1}(0)=f^{-1}(0)$ para todo $t \in[0,1]$, pois $f$ e $g$ têm o mesmo sinal em cada componente conexa de $U-f^{-1}(0)$. Além disso, a condição iii) do Lema 2.14 é satisfeita para $F_{t}$. Daí, seguindo os passos da prova do Lema 2.14 conseguimos exibir homeomorfismos que da maneira como são construídos, são homeomorfismos definíveis em $\mathcal{S}$. Logo, obtemos que $F_{t}$ e $F_{t^{\prime}}$ são topologicamente $\mathcal{K}$-equivalentes em $\mathcal{S}$, quaisquer que sejam $t, t^{\prime} \in[0,1]$. Em particular, $f$ e $g$ são topologicamente $\mathcal{K}$-equivalentes em $\mathcal{S}$.

\section{Prova do Teorema 4.3}

1. Seja $f:\left(\mathbb{R}^{n}, 0\right) \rightarrow \mathbb{R}$ um germe de função contínua definível em $\mathcal{S}$. Considere uma triangulação do par $\left(\mathbb{R}^{n}, f^{-1}(0)\right)$. Seja $(M, Y)$ o link desta triangulação em 0 . Scja $c Y$ um cone sobre $Y$ considerado como uma união de todos simplexos desta triangulação, tal que $\{0\}$ é um vértice e todos os outros vértices pertençam a $Y$. Por [15] existe um germe de homeomorfismo definível em $\mathcal{S}, h:\left(\mathbb{R}^{n}, c Y\right) \rightarrow\left(\mathbb{R}^{n}, f^{-1}(0)\right)$. Seja $Z$ uma união de todos os lados de $Y$ com codimensão maior que zero. Seja $\left\{\tilde{U}_{i}\right\}$ uma $\mathcal{K}$-decomposição correspondendo a $M$ e $Z$. Scja $\tilde{f}(x)=f(h(x))$. Seja $\alpha=\sum_{i} a_{i} T_{i}$ umla função tenda com $a_{i}=\operatorname{sinal}\left[\left.\tilde{f}\right|_{\tilde{U}_{i}}\right]$. Pelo Lema 4.4, $\tilde{f}$ e $\alpha$ são topologicamente $\mathcal{K}$-equivalentes em $\mathcal{S}$.

2. Sejam $\alpha$ e $\beta$ funções tenda associadas a diferentes $\mathcal{K}$-decomposições de $\mathbb{R}^{n}$. Suponha que $\alpha$ e $\beta$ são topologicamente $\mathcal{K}$-equivalentes. Então, por definição de $\mathcal{K}$-equivalĉncia topológica, um homeomorfismo definível em $\mathcal{S}, H:\left(\mathbb{R}^{n} \times \mathbb{R}, 0\right) \rightarrow\left(\mathbb{R}^{n} \times \mathbb{R}, 0\right)$ aplica o gráfico de $\alpha$ no gráfico de $\beta$. Como $H$ é homeomorfismo definível em $\mathcal{S}$, ele admite uma 
triangulação. Uma triangulação de $H$ fornece uma equivalência combinatorial entre $\alpha$ : $\beta$.

\subsection{Polinômios de 2 variáveis e $\mathcal{K}$-invariantes}

Uma $\mathcal{K}$-decomposição de $\mathbb{R}^{2}$ pode ser descrita como uma coleção finita de semi-retas com ponto inicial $(0,0) \in \mathbb{R}^{2}$. Os conjuntos $\tilde{U}_{i}$ são seções entre estas semi-retas. Uma classo de equivalência de funçôes tenda por uma equivalência combinatorial descrita na Seção 4.1 i uma função $\eta:\left\{\check{U}_{i}\right\} \rightarrow\{-1,0,1\}$.

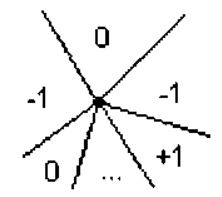

Figura 4.1:

Fin outras palavras, este invariante pode ser descrito como uma classe de equivalência de uma coleção finita de elementos $-1,0$ ou 1 por permutações cíclicas. Uma classe do equivalência $\eta$ descrita acima é chamada de $\mathcal{K}$-invariante. Un $\mathcal{K}$-invariante é chamado analítico se

1. o número de setores é par;

2. para todo $i, \eta(i) \neq 0$.

Claramente, se o germe de função $f:\left(\mathbb{R}^{n}, 0\right) \rightarrow \mathbb{R}$ ć analítico, então o $\mathcal{K}$-invariante correspondente é analítico. Dizemos que um $\mathcal{K}$-invariante $\eta$ admite uma realização algébrica se existe um polinòmio $f(x, y)$, de duas variáveis, tal que o $\mathcal{K}$-invariante do germe deste polinômio em $(0,0) \in \mathbb{R}^{2}$ é igual a $\eta$.

Teorema 4.5. Todo $\mathcal{K}$-invariante analítico admite uma realização algébrica.

Precisamos do seguinte lema.

Lema 4.6. Scjam $\gamma_{1}, \gamma_{2}:[0, c) \rightarrow \mathbb{R}^{2}$ arcos semialyébricos tais que $\gamma_{1}(0)=\gamma_{2}(0)=0$ e os vetores tangentes em $\left(0\right.$ a $\gamma_{1}$ e $\gamma_{2}$ são os mesmos. Então, existe um polinômio $p(x, y)$ tal que:

1. o conjunto $\left\{(x, y) \in \mathbb{R}^{2} \mid p(x, y)=0\right\}$ ć uma curva com dois semi-ramos $\tilde{\gamma}_{1}$ e $\tilde{\gamma}_{2}$; 
2. a curva $\left\{(x, y) \in \mathbb{R}^{2} \mid p(x, y)=0\right\}$ tem um ponto singular em $(0,0) \in \mathbb{R}^{2}$;

3. os vetores unitários tangentes a $\tilde{\gamma}_{1}$ e $\tilde{\gamma}_{2}$ em $(0,0) \in \mathbb{R}^{2}$ são os mesmos e iguais ao vetor unitário tangente a $\gamma_{1}$ em $(0,0) \in \mathbb{R}^{2}$;

4. para pequenos valores de $t \neq 0$, temos $p\left(\gamma_{1}(t)\right)>0$ e $p\left(\gamma_{2}(t)\right)>0$

5. o conjunto $\left\{(x, y) \in \mathbb{R}^{2} \mid p(x, y) \leq 0\right\}$ é limitado pelas curvas $\tilde{\gamma}_{1}$ e $\tilde{\gamma_{2}}$

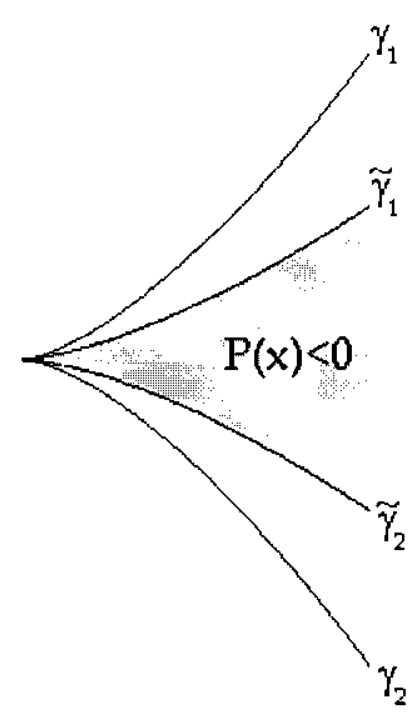

Figura 4.2:

Prova. Podemos aplicar um blowing-up em $(0,0) \in \mathbb{R}^{2}$ várias vezes e obter uma figura $X$ onde as imagens inversas das curvas $\gamma_{1}$ e $\gamma_{2}$ terão vetores tangentes diferentes. Seja $\pi: X \rightarrow \mathbb{R}^{2}$ uma composição destes blowing-ups, onde $X$ é uma variedade bi-dimensional. Seja $\tilde{\gamma}_{i}=\pi^{-1} \gamma_{i}(i=1,2)$ as imagens inversas destes arcos. Como $\tilde{\gamma}_{1}$ e $\tilde{\gamma}_{2}$ têm vetores tangentes diferentes em $\tilde{\gamma}_{1}(0)=\tilde{\gamma}_{2}(0)=x_{0}$, existe uma curva algébrica real $\beta$ com uma singularidade cuspidal tal que $\beta$ está contida na área localmente limitada por $\tilde{\gamma}_{1}$ e $\tilde{\gamma}_{2}$ eo ponto singular de $\beta$ é igual a $x_{0}$ (Figura 4.3 ).

Seja $\tilde{p}(\tilde{x})=0$ uma equação algébrica de $\beta$ tal que $\tilde{p}(\tilde{x})<0$ na área cuspidal. Aplicando $\pi$ na função $\tilde{p}$, obtemos uma função $p$ satisfazendo às afirmações do lema.

Dizemos que um $\mathcal{K}$-invariante analítico $\eta$ é alternado se, para cada $i, \eta(i) \neq \eta(i+1)$

Afirmação 1. Seja $\eta$ um $\mathcal{K}$-invariante analítico alternado. Então $\eta$ admile a seguinte realização algébrica:

$$
p(x, y)=(x+y)(2 x+y) \cdots(s x+y) .
$$




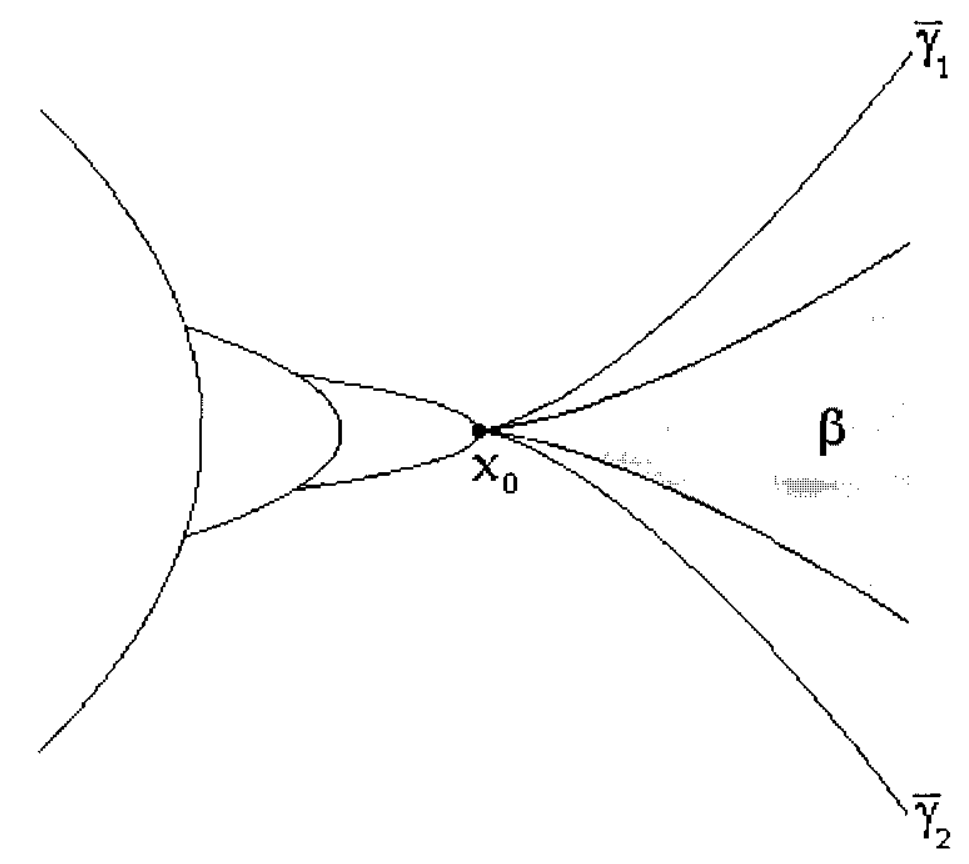

Figura 4.3:

Prova. O conjunto $\left\{(x, y) \in \mathbb{R}^{2} \mid p(x, y)=0\right\}$ é uma coleção de retas dadas por $l_{i}=\left\{(x, y) \in \mathbb{R}^{2}: i x+y=0\right\}$. Sejam $\tilde{U}_{i}, \tilde{U}_{i \dagger 1}$ dois setores tais que $\tilde{U}_{i} \cap \tilde{U}_{i+1}$ seja a reta $l_{i}$. Observe que a função $i x+y$ tem diferentes sinais em $\operatorname{int}\left(\tilde{U}_{i}\right)$ e em $\operatorname{int}\left(\tilde{U}_{i+1}\right)$. Então, a função $p$ também tem diferentes sinais em $\operatorname{int}\left(\tilde{U}_{i}\right)$ e em $\operatorname{int}\left(\tilde{U}_{i+1}\right)$. Então, como o $\mathcal{K}$-invariante de $p$ é alternado, $\eta$ admite a realização algébrica $p(x, y)=(x+y)(2 x+y) \cdots(s x+y)$. $\square$

Um $\mathcal{K}$-invariante analítico $\eta$ ć chamado duplamente alternado sc, para cada $i, \eta(i) \neq$ $\eta(i+2)$. Por exemplo, a sequência

$$
1,1,-1,-1,1,1 \ldots
$$

Afirmação \%. Um K $\mathcal{K}$-invariante analítico duplamente alternado admile a seguinte realização algébrica

$$
p(x, y)=(x+y)(2 x+y)^{2}(3 x+y)(4 x+y)^{2} \cdots(2 s x+y)^{2} .
$$

Prova. Seja $\theta \in S^{1}$. Então, para todo $r>0$, temos que sinal $[p(r \theta)]=\operatorname{sinal}[p(\theta)]$. Suponha que $\theta$ se move ao longo de $S^{1}$. Então, $p(\theta)$ não muda de sinal quando $\theta$ cruza um ponto $l_{i} \cap S^{1}$, para $i$ par, e $p(\theta)$ muda de sinal quando $\theta$ cruza um ponto $l_{i} \cap S^{\prime}$, para $i$ ímpar, onde $l_{i}:=i x+y=0$ (Figura 4.4). Então, esta configuração admite uma realização algébrica do tipo 


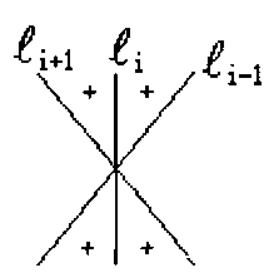

Figura 4.4:

$$
p(x, y)=(x+y)(2 x+y)^{2}(3 x+y)(4 x+y)^{2} \cdots(2 s x+y)^{2} .
$$

Prova do Teorema 4.5 Observe que o número de setores, para um $\mathcal{K}$-invariante analítico, é par, ou seja, $2 r$, para algum inteiro $r>0$. Lsaremos indução sobre $r$. Se $r=1$ a afirmação é trivial. De fato, a sequência $(-1,1)$ é realizada, por cxcmplo, por uma função $p(x, y)=x$; a sequência $(1,1)$ é realizada por $p(x, y)=x^{2}$ c a sequência $(-1,-1)$ é realizada por $p(x, y)=-x^{2}$. Suponha que todos os $\mathcal{K}$-invariantes, para todo $r \leq r_{0}$ sejam algebricamente realizados. Considere agora uma sequência com $2 r_{0}+2$ clementos. Se a sequência é duplamente alternada então, ela é realizada (ver Afirmação 2). Então podemos supor que a sequência não é duplamente alternada. Daí, existem três elementos consecutivos $\eta(i), \eta(i+1), \eta(i+2)$ tais que $\eta(i)=\eta(i+2)$. Considere outro $\mathcal{K}$-invariante obtido deste primciro substituindo estes elementos consecutivos por apenas $\eta(i)$ como na Figura 4.5:
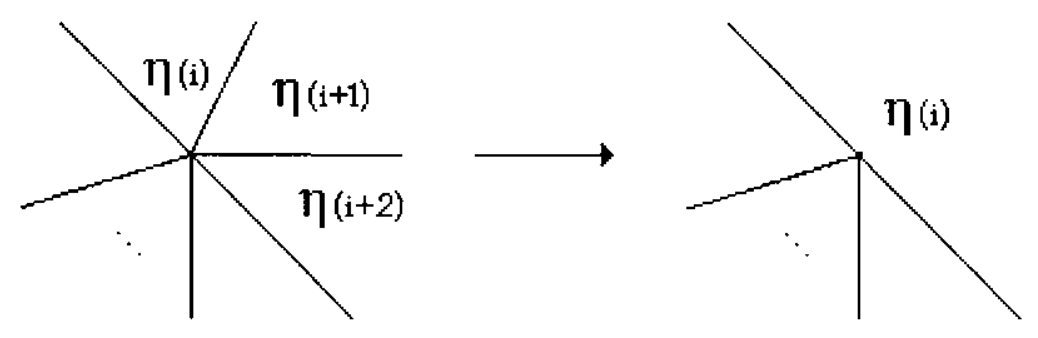

Figura 4.5:

Por hipótese de indução, a nova configuração ć algebricamente realizável. Seja $p_{1}(x, y)$ um germe de um polinômio real realizando este novo $\mathcal{K}$-invariante. Sejam $\gamma_{1}$ e $\gamma_{2}$ arcos semialgébricos limitando a área correspondente a $\eta(i)$. Pelo Lema 4.6, existe um polinômio $p(x, y)$ satisfazendo as condições do lema sendo positivo fora da área limitada pela curva $\left\{(x, y) \in \mathbb{R}^{2} \mid p(x, y)=0\right\}$. Então, a rcalização $\tilde{p}$ do $\mathcal{K}$-invariante pode ser obtida pelo produto $\tilde{p}=p_{1} . p$. 
Corolário 4.7. Seja $f(x, y)$ uma função analítica em $(0,0) \in \mathbb{R}^{2}$. Lntâo, existe um polinômio $p(x, y)$ tal que o germe de $f$ em $(0,0) \in \mathbb{R}^{2}$ é topologicamente $\mathcal{K}$-equivalente ao germe de $p$ em $(0,0) \in \mathbb{R}^{2}$.

O seguinte teorena fornece uma forma normal para todos os germes de funções em duas variáveis $\mathcal{C}^{0}-\mathcal{K}$-finitamente determinados.

Teorema 4.8. Seja $f:\left(\mathbb{R}^{2}, 0\right) \rightarrow(\mathbb{R}, 0)$ um germe de função definivel em $\mathcal{S}, \mathcal{C}^{0}-\mathcal{K}$-finitamente determinado. Então, $f$ é topologicamente $\mathcal{K}$-equivalente a um produto de funções lineares

$$
p(x, y)=(x+y)(2 x+y) \cdots(s x+y) .
$$

Prova. Como $f \dot{e} \mathcal{C}^{0}-\mathcal{K}$-finitamente determinado, $f^{-1}(0) \cap \Sigma f=\{0\}$ (Proposição 2.6). Seja $\gamma$ um semi-ramo da curva $\left\{(x, y) \in \mathbb{R}^{2} \mid f(x, y)=0\right\}$. Como $(\gamma-\{0\}) \cap \Sigma f=\emptyset$, a função $f$ muda de sinal quando cruza $\gamma$. Logo, o $\mathcal{K}$-invariante de $f$ é alternado. Pela Afirmação 1 , segue que $f$ é topologicamente $\mathcal{K}$-equivalente a um produto de funções lineares

$$
p(x, y)=(x+y)(2 x+y) \cdots(s x+y)
$$

Dado um germe de função $f:\left(\mathbb{R}^{2}, 0\right) \rightarrow(\mathbb{R}, 0)$, o conjunto $B_{\varepsilon}^{2} \cap f^{-1}(0)-\{0\}, \varepsilon>0$, é em gcral vazio ou uma união de curvas suaves, as quais chamamos semi-ramos de $f^{-1}(0)$. O número de semi-ramos coincide com o número de componentes conexas do link $S_{\varepsilon}^{1} \cap f^{-1}(0)$ e independe da escolha do representante do germe $f$. O corolário a seguir mostra que o número de semi-ramos é um invariante completo para a $\mathcal{C}^{0}$ - $\mathcal{K}$-equivalência de germes de funções $\left(\mathbb{R}^{2}, 0\right) \rightarrow(\mathbb{R}, 0), \mathcal{C}^{0}-\mathcal{K}$-finitos.

Corolário 4.9. Dois germes $\mathcal{C}^{0}-\mathcal{K}$-finitamente determinados são topologicamente $\mathcal{K}$-equivalentes se, e somente se, seus conjuntos de zeros têm o mesmo número de semi-ramos.

\subsection{Interpretação geométrica}

Conforme vimos na Definição 2.25 , dadas $\mathcal{X}_{i}, \mathcal{Y}_{i}, i=1,2$, subvariedades do $\mathbb{R}^{n}$, dizemos que $\left(\mathcal{X}_{1}, \mathcal{Y}_{1}\right)$ e $\left(\mathcal{X}_{2}, \mathcal{Y}_{2}\right)$ têm o mesmo tipo de contato topológico na origem se existe um germe de homeomorfismo $H:\left(\mathbb{R}^{n} ; 0\right) \rightarrow\left(\mathbb{R}^{n}, 0\right)$ tal que $H\left(\mathcal{X}_{1}\right)=\mathcal{X}_{2}$ e $H\left(\mathcal{Y}_{1}\right)=\mathcal{Y}_{2}$, e a notação usada para isto é

$$
\mathcal{C}^{0}-K\left(\mathcal{X}_{1}, \mathcal{Y}_{1}, 0\right)=\mathcal{C}^{0}-K\left(\mathcal{X}_{2}, \mathcal{Y}_{2}, 0\right)
$$


Para o caso de hipersuperfícies, temos o seguinte resultado:

Teorema 4.10. Sejam $\mathcal{X}_{i}, \mathcal{Y}_{i}$ subvariedades do $\mathbb{R}^{n}$ tais que dim $\mathcal{X}_{i}=\operatorname{dim} \mathcal{Y}_{i}=n-1$, $i=1$, 2 . Sejam $g_{i}:\left(\mathcal{X}_{i}, 0\right) \longrightarrow\left(\mathbb{R}^{n}, 0\right)$ germes de imersões e $f_{i}:\left(\mathbb{R}^{n}, 0\right) \longrightarrow(\mathbb{R}, 0)$ germes de submersões com $f_{i}^{-1}(0)=\mathcal{Y}_{i}, i=1,2$. Então,

$\mathcal{C}^{0}-K\left(\mathcal{X}_{1}, \mathcal{Y}_{1}, 0\right)=\mathcal{C}^{0}-K\left(\mathcal{X}_{2}, \mathcal{Y}_{2}, 0\right) \Longleftrightarrow f_{1} \circ g_{1} \quad e \quad f_{2} \circ g_{2} \quad$ são $\quad \mathcal{C}^{0}-\mathcal{X}$-equivalentes

Prova.

$\Leftrightarrow$ Como $\mathcal{C}^{0}-K\left(\mathcal{X}_{1}, \mathcal{Y}_{1}, 0\right)=\mathcal{C}^{0}-K\left(\mathcal{X}_{2}, \mathcal{Y}_{2}, 0\right)$, existe um homeomorfismo $H$ tal que

$$
\Pi\left(\mathcal{X}_{1}\right)=\mathcal{X}_{2} \text { e } H\left(\mathcal{Y}_{1}\right)=\mathcal{Y}_{2}
$$

Observe que $H\left(\mathcal{X}_{1} \cap \mathcal{Y}_{1}\right)=\mathcal{X}_{2} \cap \mathcal{Y}_{2}$, ou seja, $\mathcal{X}_{1} \cap \mathcal{Y}_{1}$ é homeomorfo a $\mathcal{X}_{2} \cap \mathcal{Y}_{2}$. Para indicar este homeomorfismo usaremos a notação $\stackrel{\text { homeo }}{\cong}$. Alćm disso, fazendo as devidas identificações e lembrando que $\mathcal{X}_{i}$ é dada parametrizada por $g_{i}$, segue que

$$
\left(f_{1} \circ g_{1}\right)^{-1}(0)=g_{1}^{-1}\left(\mathcal{X}_{1} \cap \mathcal{Y}_{1}\right) \stackrel{\text { diffco }}{\cong} \mathcal{X}_{1} \cap \mathcal{Y}_{1} \stackrel{\text { homneo }}{\cong} \mathcal{X}_{2} \cap \mathcal{Y}_{2} \stackrel{\text { difeo }}{\cong} g_{2}^{-1}\left(\mathcal{X}_{2} \cap \mathcal{Y}_{2}\right)=\left(f_{2} \circ g_{2}\right)^{-1}(0),
$$

onde $\stackrel{\text { difeo }}{\cong}$ indica que os conjuntos são difeomorfos. $\Lambda$ ssim,

$$
g_{2}^{-1} \circ H \circ g_{1}\left(\left(f_{1} \circ g_{1}\right)^{-1}(0)\right)=\left(f_{2} \circ g_{2}\right)^{-1}(0) .
$$

Portanto, $f_{1} \circ g_{1}$ e $f_{2} \circ g_{2}$ são $\mathcal{C}^{0}-\mathcal{V}$-cquivalentes. Então, pelo Corolário 2.16, $f_{1} \circ g_{1} \mathrm{c}$ $f_{2} \circ g_{2}$ são $\mathcal{C}^{0}-\mathcal{K}$-equivalentes.

$(\Leftarrow)$ Por hipótese $\operatorname{dim} \mathcal{X}_{i}-\operatorname{dim} \mathcal{Y}_{i}=n-1=k$. A idéia é tentar expressar $\mathcal{X}_{i}$ como gráfico da aplicação $\phi_{i}: \mathbb{R}^{k} \longrightarrow \mathbb{R}$ e $\mathcal{Y}_{i}$ como gráfico da aplicação nula de $\mathbb{R}^{k}$ em $\mathbb{R}$. Para isso, escolhemos um sistema de coordenadas para $\mathbb{R}^{n}$ tal que

$$
f_{1}\left(x_{1}, \ldots, x_{n}\right)=x_{n}
$$

Assim, $\mathcal{Y}_{1}=f_{1}^{-1}(0)=\mathbb{R}^{k} \times\{0\}$. Agora, escolha um subespaço $V_{1}$, 1-dimensional, transversal a $\mathcal{X}_{1}$ e a $\mathcal{Y}_{1}$, tal que $\mathbb{R}^{n}=\mathcal{Y}_{1} \times V_{1}$.

Defina $\pi: \mathbb{R}^{n}=\mathcal{Y}_{1} \times V_{1} \longrightarrow \mathcal{Y}_{1}$ a projeção no primciro fator. Assim,

$$
\left.\pi\right|_{\mathcal{X}_{1}}: \mathcal{X}_{1} \subset \mathbb{R}^{n} \longrightarrow \mathcal{Y}_{1}
$$

é um difeomorfismo o qual induz um sistema de coordenadas em $\mathcal{X}_{1}$.

Com respeito a esse sistema de coordenadas $\mathcal{Y}_{1}$ é o gráfico da aplicação nula e $\mathcal{X}_{1}$ é o gráfico de $f_{1} \circ g_{1}$, pensando em $f_{1}$ como a projeção no segundo fator: $\mathcal{Y}_{1} \times V_{1} \longrightarrow V_{1}$.

Analogamente, podemos fazer a mesma construção para $\mathcal{X}_{2}$ e $\mathcal{Y}_{2}$. 
Como por hipótese temos que $f_{1} \circ g_{1}$ e $f_{2} \circ g_{2}$ são $\mathcal{C}^{0}$ - $\mathcal{K}$-equivalentes, sabemos por definição que existe um germe de homeomorfismo $H:\left(\mathbb{R}^{k} \times \mathbb{R}, 0\right) \longrightarrow\left(\mathbb{R}^{k} \times \mathbb{R}, 0\right)$ tal que $H\left(\mathbb{R}^{k} \times\{0\}\right)=\mathbb{R}^{k} \times\{0\}$ e $H$ aplica o gráfico de $f_{1} \circ g_{1}$ no gráfico de $f_{2} \circ g_{2}$, ou seja, existe um homeomorfismo $H$ tal que $H\left(\mathcal{Y}_{1}\right)=\mathcal{Y}_{2}$ e $H\left(\mathcal{X}_{1}\right)=\mathcal{X}_{2}$.

Portanto, $\mathcal{C}^{0}-K\left(\mathcal{X}_{1}, \mathcal{Y}_{1}, 0\right)=\mathcal{C}^{0}-K\left(\mathcal{X}_{2}, \mathcal{Y}_{2}, 0\right)$.

Muitas vezes, nossa análise do contato topológico entre subvariedades do $\mathbb{R}^{N}$, se restringirá apenas aos pares de subvariedades do tipo (gráfico de $f, \mathbb{R}^{n} \times\{0\}$ ), onde $f:\left(\mathbb{R}^{n}, 0\right) \rightarrow\left(\mathbb{R}^{p}, 0\right)$. Nestes casos, quando nos referimos à versão topológica dà definição de contato proposta por Mather-Montaldi, queremos dizer que existe um germe de homeomorfismo $H:\left(\mathbb{R}^{n} \times \mathbb{R}^{p}, 0\right) \rightarrow\left(\mathbb{R}^{n} \times \mathbb{R}^{p}, 0\right)$ tal que

$$
H(\operatorname{graf}(f))=\operatorname{graf}(g) \text { e } H\left(\mathbb{R}^{n} \times\{0\}\right)=\mathbb{R}^{n} \times\{0\}
$$

ou

$$
\mathcal{C}^{0}-K\left(\operatorname{graf}(f), \mathbb{R}^{n} \times\{0\}, 0\right)=\mathcal{C}^{0}-K\left(\operatorname{gral}(g), \mathbb{R}^{n} \times\{0\}, 0\right) .
$$

A proposição a seguir é uma versão topológica do Corolário 1.52. Fla afirma que, para germes $\mathcal{C}^{0}-\mathcal{K}$-finitos, basta um homeomorfismo que leva gráfico no gráfico e preserva o $\mathbb{R}^{n} \times\{0\}$, para obtermos a $\mathcal{C}^{0}-\mathcal{K}$-equivalência. Logo, temos uma versão topológica dos resultados de Mather-Montaldi:

Proposição 4.11. Sejam $f, g:\left(\mathbb{R}^{n}, 0\right) \longrightarrow(\mathbb{R}, 0), n \geq 2$, germes $\mathcal{C}^{0}$-K - finitamente determinados. Então, $\mathcal{C}^{0}-K\left(\operatorname{graf}(f), \mathbb{R}^{n} \times\{0\}, 0\right)=\mathcal{C}^{0}-K\left(\operatorname{graf}(g), \mathbb{R}^{n} \times\{0\}, 0\right)$ se, e somente se, $f$ e g são $\mathcal{C}^{0}-\mathcal{K}$-equivalentes.

Prova. Suponha $f$ e $g$ germes tais que

$$
\mathcal{C}^{0}-K\left(\operatorname{graf}(f), \mathbb{R}^{n} \times\{0\}, 0\right)=\mathcal{C}^{0}-K\left(\operatorname{graf}(g), \mathbb{R}^{n} \times\{0\} ; 0\right),
$$

isto é, existe um germe de homeomorfismo $H:\left(\mathbb{R}^{n} \times \mathbb{R}, 0\right) \rightarrow\left(\mathbb{R}^{n} \times \mathbb{R}, 0\right)$ tal que $H(\operatorname{graf}(f))=\operatorname{graf}(g)$ e $H\left(\mathbb{R}^{n} \times\{0\}\right)=\mathbb{R}^{n} \times\{0\}$.

Como $I I(\operatorname{graf}(f))=\operatorname{graf}(g)$, temos que, em particular, existe um homeomorfismo $h$ do $\mathbb{R}^{n}$ tal que $h\left(f^{-1}(0)\right)=g^{-1}(0)$. Como $f$ (resp. $g$ ) e $\mathcal{C}^{0}-\mathcal{K}$-finito, existe uma vizinhança $U$ de 0 em $\mathbb{R}^{n}$ tal que qualquer ponto de $f^{-1}(0) \cap U-\{0\}$ (resp. $g^{-1}(0) \cap U-\{0\}$ ) é regular. Daí, como germes na origem, segue que uma das seguintes condiçôs são satisfeitas:

a) sinal $[f(x)]=\operatorname{sinal}[g \circ h(x)]$ para todo $x \in \mathbb{R}^{n}-f^{-1}(0)$, ou

b) sinal $[f(x)]-\operatorname{sinal}[-g \circ h(x)]$ para todo $x \in \mathbb{R}^{n}-f^{-1}(0)$. 
Suponha que a) ocorra. Construa a homotopia linear $F_{t}(x)=(1-t) f(x)+\operatorname{tg}(h(x))$, $t \in[0,1]$. Daí, $F_{t}$ satisfaz as hipóteses do Lcma 2.14. Logo, $f$ e $g$ são $\mathcal{C}^{0}-\mathcal{K}$-equivalentes. A recíproca ć imediata.

Mesmo na linguagem das estruturas o-minimais, o 'Teorema 4.3 também pode ser interpretado como uma versão topológica dos resultados de Mather-Montaldi. De fato, considere $\mathcal{S}$ uma estrutura o-minimal sobre $\mathbb{R}$. De modo análogo ao feito nas Definiçôes 1.50 e 2.25 , podemos definir quando duas subvariedades definíveis em $\mathcal{S}, \mathcal{X}, \mathcal{Y} \subset \mathbb{R}^{n}$, têm o mesmo tipo definível de contato topológico na origem. Ou seja, dizemos que os pares de subvariedades definíveis em $\mathcal{S},\left(\mathcal{X}_{1}, \mathcal{Y}_{1}\right)$ e $\left(\mathcal{X}_{2}, \mathcal{Y}_{2}\right)$ têm o mesmo tipo definivel de contato topológico na origem se existe um germe de homeomorfismo definível em $\mathcal{S}$ $H:\left(\mathbb{R}^{n}, 0\right) \rightarrow\left(\mathbb{R}^{n}, 0\right)$ tal que $H\left(\mathcal{X}_{1}\right)=\mathcal{X}_{2}$ e $H\left(\mathcal{Y}_{1}\right)=\mathcal{Y}_{2}$.

Em outras palavras, o Teorema 4.3 pode ser interpretado da seguinte forma: dois germes de função definíveis em $\mathcal{S}, f, g:\left(\mathbb{R}^{n}, 0\right) \rightarrow \mathbb{R}$ são $\mathcal{C}^{0}-\mathcal{K}$-equivalentes se, e somente se, os pares (graf $\left.(f), \mathbb{R}^{n} \times\{0\}\right)$ e (graf $\left.(g), \mathbb{R}^{n} \times\{0\}\right)$ têm o mesmo tipo definívol de contato topológico na origem. 


\title{
Capítulo 5
}

\section{$\mathcal{C}^{0}$ - $\mathcal{K}$-equivalência de aplicações}

\author{
Caso $n \geq p$
}

Neste capítulo, queremos estudar o que ocorre com a $\mathcal{C}^{0}-\mathcal{K}$-equivalência dos germes $f:\left(\mathbb{R}^{n}, 0\right) \rightarrow\left(\mathbb{R}^{p}, 0\right)$ com $n \geq p$, principalmente quando $p \neq 1$, pois o caso de funções foi tratado separadamente no Capítulo 4 . Um dos objetivos é mostrar em que casos a $\mathcal{C}^{0}-\mathcal{V}$-equivalência implica na $\mathcal{C}^{0}-\mathcal{K}$-equivalência. Este resultado se torna especialmente importante quando for possível construir um conjunto completo de invariantes para a $\mathcal{C}^{0}$ - $\mathcal{V}$-equivalência. Vimos que nos casos $n=p$ e para germes de funções $(p=1)$, já temos resposta para esta questão. Mas, considerando qualquer par de dimensão $(n, p)$, este é um problema que se torna mais difícil a medida que a dimensão do link da singularidade cresce. O caso mais natural para dar sequência a cstc estudo é quando $p=n-1$, pois o germe $f:\left(\mathbb{R}^{n}, 0\right) \rightarrow\left(\mathbb{R}^{n-1}, 0\right)$ define um germe de curva $X=f^{-1}(0)$. A topologia de $X$ é um dos problemas clássicos da teoria de singularidades.

As respostas que obtivemos são parciais. De um modo geral, o caso $n>p \neq 1$ ainda está incompleto. Dividimos este capítulo em duas partes. Na primeira, mostramos o que obtivemos para os germes de curvas $(p=n-1)$; na segunda, apresentamos os principais resultados do capítulo, estudando o que ocorre com a $\mathcal{C}^{0}-\mathcal{K}$-equivalôncia para famílias de germes de aplicações com $n \geq p$.

\subsection{Caso de curvas $(p=n-1)$}

Considere um germe $f:\left(\mathbb{R}^{n}, 0\right) \rightarrow\left(\mathbb{R}^{n-1}, 0\right)$. Dado $\varepsilon>0$, o conjunto $B_{\varepsilon}^{n} \cap f^{-1}(0)-\{0\} \dot{e}$ em geral vazio ou uma união de curvas suaves, as quais chamamos semi-ramos de $f^{-1}(0)$. O número de semi-ramos de $f^{-1}(0)$ em 0 coincide com o número de componentes conexas 
do link $f^{-1}(0) \cap S_{\varepsilon}^{n-1}$ e independe da escolha do representante do germe $f$. Conjecturamos que o número de semi-ramos da curva $X=f^{-1}(0)$ é um invariante completo da $\mathcal{C}^{0}-\mathcal{K}$ equivalĉncia. Para $n=2$, isto é verificado no Corolário 4.9. Para os demais casos, a idéia desta conjectura surge a partir da leitura do artigo [2]. Não temos ainda a prova no geral, mas apresentamos alguns casos onde ela é verdadeira.

Dado um germe de aplicação $f=\left(f_{1}, \ldots, f_{n-1}\right):\left(\mathbb{R}^{n}, 0\right) \rightarrow\left(\mathbb{R}^{n-1}, 0\right)$ considere o germe

$$
\left(f_{1}, \ldots, f_{n-1}, x_{1}^{2}+\ldots+x_{n}^{2}\right):\left(\mathbb{R}^{n}, 0\right) \rightarrow\left(\mathbb{R}^{n}, 0\right)
$$

e o conjunto $\Delta=\operatorname{det}\left(\frac{\partial\|x\|^{2}}{\partial x}, \frac{\partial f}{\partial x}\right)$, onde $x=\left(x_{1}, \ldots, x_{n}\right)$ é um sistema de coordenadas locais de $\mathbb{R}^{n}$ na origem e $\|x\|^{2}=x_{1}^{2}+\ldots+x_{n}^{2}$.

Então, obtemos um novo germe de aplicação

$$
(f, \Delta):\left(\mathbb{R}^{n}, 0\right) \rightarrow\left(\mathbb{R}^{n}, 0\right) .
$$

Se $(f, \Delta)^{-1}(0)=\{0\}$ como germe, podemos definir o grau de $(f, \Delta)$.

Teorema 5.1. (Aoki-Fukuda-Nishimura [2]) Suponha que $f:\left(\mathbb{R}^{n}, 0\right) \rightarrow\left(\mathbb{R}^{n-1}, 0\right)$ é um germe analítico genérico no sentido de que $(f, \Delta)^{-1}(0)=\{0\}$. Então, o número de semiramos de $f^{-1}(0)$ é igual a $2|\operatorname{grau}(f, \Delta)|$, onde $|\operatorname{grau}(f, \Delta)|$ é o valor absoluto do grau de $(f, \Delta)$.

Novamente usando Eisenbud-Levine $|16|$ podemos calcular $|\operatorname{grau}(f, \Delta)|$ em termos da $\mathbb{R}$-álgebra local $Q(f, \Delta)$ associada ao germe $(f, \Delta):\left(\mathbb{R}^{n}, 0\right) \rightarrow\left(\mathbb{R}^{n}, 0\right)$. Então temos:

Teorema 5.2. (Aoki-Fukuda-Nishimura [2]) Seja $f:\left(\mathbb{R}^{n}, 0\right) \rightarrow\left(\mathbb{R}^{n-1}, 0\right)$ um germe analítico tal que $\operatorname{dim}_{\mathbb{R}} Q(f, \Delta)<\infty$. Então, o número de semi-ramos de $f^{-1}(0)$ é igual a

$$
2\left\{\operatorname{dim}_{\mathbb{R}} Q(f, \Delta)-2 \operatorname{dim}_{\mathbb{R}} I(f, \Delta)\right\}
$$

onde $I=I(f, \Delta)$ é um ideal de $Q(f, \Delta)$ o qual é maximal com respeito a propriedade $I^{2}=0$.

É óbvio que se $\operatorname{dim}_{\mathbb{R}} Q(f, \Delta)<\infty$, então $(f, \Delta)^{-1}(0)=\{0\}$. A recíproca nem sempre é verdadeira.

Z. Szafraniec [59] generaliza o Teorema 5.1 da seguinte forma:

Teorema 5.3. (Szafraniec [59]) Sejam $f:\left(\mathbb{R}^{n}, 0\right) \rightarrow\left(\mathbb{R}^{n-1}, 0\right)$ e $\xi:\left(\mathbb{R}^{n}, 0\right) \rightarrow(\mathbb{R}, 0)$ germes analíticos tais que $f^{-1}(0)$ define um germe de curva em $\mathbb{R}^{n}$ e $\xi$ não se anula em $f^{-1}(0)-\{0\}$. Sejam 
$b_{+}-$o número de componentes conexas de $f^{1}(0) \cap\{\xi>0\}, e$

$b_{-}=$o numero de componentes conexas de $f^{-1}(0) \cap\{\xi<0\}$.

Então, $b_{+}-b_{-}-2 \operatorname{grau}(J, f)$, onde $J=\operatorname{det}\left(\frac{\partial \xi}{\partial x}, \frac{\partial f}{\partial x}\right)$, e $x=\left(x_{1}, \ldots, x_{n}\right)$ é umr sistema de coordenadas locais de $\mathbb{R}^{n}$ na origem.

Recorde que estamos interessados em mostrar as situaçoes nas quais o número de semi-ramos de um germe de curva ć um invariante completo da $\mathcal{C}^{0}$ - $\mathcal{K}$-equivalência. Na proposição a seguir, apresentamos um caso muito particular, no qual a curva não possui semi-ramos. Vejamos o que acontece quando $f^{-1}(0)=g^{-1}(0)=\{0\}$.

Proposição 5.4. Sejam $f, g:\left(\mathbb{R}^{n}, 0\right) \rightarrow\left(\mathbb{R}^{n-1}, 0\right)$ germes reais analíticos tais que $f^{-1}(0)=$ $g^{-1}(0)=\{0\}$. Então, $f$ é $\mathcal{C}^{0}-\mathcal{K}$-equivalente a $g$.

Prova. Considere $\xi:\left(\mathbb{R}^{n}, 0\right) \rightarrow(\mathbb{R}, 0)$ dado por $\xi=f_{1}^{2}+\ldots+f_{n-1}^{2}$. Observe que $\xi^{\cdots 1}(0)=\{0\}$ e $\xi(x)>0$, para todo $x \neq 0$ em $\mathbb{R}^{n}$. Seja $J=\operatorname{det}\left(\frac{\partial \xi}{\partial x} ; \frac{\partial f}{\partial x}\right)$ como no Teorema 5.3. Assim, $(f, J) \cdots(f, 0):\left(\mathbb{R}^{n}, 0\right) \rightarrow\left(\mathbb{R}^{n}, 0\right),(f, J)^{-1}(0)=\{0\}$ e pelo Teorema 5.1, temos que gran $(f, J)=\operatorname{gran}(f, 0)=0$. Podemos fazer o mesmo para $g$ e concluir que

$$
\operatorname{grau}(g, 0)=0=\operatorname{grau}(f, 0)
$$

Então, pelo l'corema $2.17,(f, 0)$ é $\mathcal{C}^{0}-\mathcal{K}$-equivalente a $(g, 0)$. Ou seja, existe um par de homeomorfismos $H:\left(\mathbb{R}^{n} \times\left[\mathbb{R}^{n-1} \times \mathbb{R}\right], 0\right) \rightarrow\left(\mathbb{R}^{n} \times\left[\mathbb{R}^{n-1} \times \mathbb{R}\right], 0\right)$ e $h:\left(\mathbb{R}^{n}, 0\right) \rightarrow\left(\mathbb{R}^{n}, 0\right)$, nas condições da Definição 2.1, tal que

$$
H(x, f(x), 0)=(h(x), g(h(x)), 0) \text { e } H(x, 0,0)=(h(x), 0,0)
$$

com $M(x, y, t)=(h(x), 0(x, y, t))$, onde $x \in \mathbb{R}^{n}, y \in \mathbb{R}^{n-1}$ e $t \in \mathbb{R}$.

Considere $H_{1}:\left(\mathbb{R}^{n} \times \mathbb{R}^{n-1}, 0\right) \rightarrow H\left(\left(\mathbb{R}^{n} \times \mathbb{R}^{n-1} \times\{0\}, 0\right)\right)$ dado por

$$
H_{1}(x, y)=H(x, y, 0)
$$

Observe que

$$
H_{1}(x, f(x))=(h(x), g(h(x)), 0), \quad H_{1}(x, 0,0)=(h(x), 0,0)
$$

e $H\left(\mathbb{R}^{n} \times \mathbb{R}^{n \cdots 1} \times\{0\}, 0\right)$ é homcomorfo a $\left(\mathbb{R}^{n} \times \mathbb{R}^{n-1}, 0\right)$. Seja $H_{2}$ um homeomorfismo entre $H\left(\mathbb{R}^{n} \times \mathbb{R}^{n-1} \times\{0\}, 0\right)$ e $\left(\mathbb{R}^{n} \times \mathbb{R}^{n-1}, 0\right)$ tal que restrito ao graf $(g)$ e restrito a $\left(\mathbb{R}^{n} \times\{0\}, 0\right)$ seja a identidade. Então, $H_{1} \circ H_{2}$ é um homeomorfismo do $\left(\mathbb{R}^{n} \times \mathbb{R}^{n-1}, 0\right)$ que aplica graf $(f)$ no graf $(g)$ e deixa o $\mathbb{R}^{n} \times\{0\}$ invariante. Logo, $f$ e $g$ são $\mathcal{C}^{0}-\mathcal{K}$-equivalentes. 
Exemplo 5.5. Os germes $f(x, y, z)=\left(x, y^{2}+z^{2}\right)$ e $g(x, y, z)=\left(x^{2}, y^{2}+z^{2}\right)$ são $\mathcal{C}^{0}-\mathcal{K}$ equivalentes. De fato, o argumento segue da Proposição 5.4. Note que como germes em uma variável real, $x$ e $x^{2}$ não são $\mathcal{C}^{0}-\mathcal{K}$-equivalentes.

Proposição 5.6. Sejam $f, g:\left(\mathbb{R}^{n}, 0\right) \rightarrow\left(\mathbb{R}^{n \cdot 1}, 0\right), n>2$, germes analíticos de coposto $\leq 1, \mathcal{C}^{0}-\mathcal{K}$-finitos tais que $f^{-1}(0)=g^{-1}(0)$. Então, $f$ e $g$ são $\mathcal{C}^{0}-\mathcal{K}$-equivalentes.

Prova. De fato, seja $x=\left(x_{1}, \ldots, x_{n}\right)$ um sistema de coordenadas con $\mathbb{R}^{n}$. Dividiremos a prova $\mathrm{cm}$ três casos:

Caso 1. Se f e g têm coposto O.

Então, $f$ e $g$ são submersões. Logo são $\mathcal{K}$-equivalentes $e$, portanto, $\mathcal{C}^{0}$-K $\mathcal{K}$-equivalentes.

Caso II. Se f e g têm coposto 1 .

Então, podemos assumir

$$
f\left(x_{1}, \ldots, x_{n}\right)=\left(x_{1}, \ldots, x_{n-2}, f_{n-1}\left(x_{n-1}, x_{n}\right)\right)
$$

e

$$
g\left(x_{1}, \ldots, x_{n}\right)=\left(x_{1}, \ldots, x_{n-2}, g_{n-1}\left(x_{n-1}, x_{n}\right)\right) .
$$

Como os germes $f$ e $g$ são $\mathcal{C}^{0}-\mathcal{K}$-finitos e $f^{-1}(0)=g^{-1}(0)$, segue que os germes $f_{n-1}, g_{n-1}:\left(\mathbb{R}^{2}, 0\right) \rightarrow(\mathbb{R}, 0)$ são também $\mathcal{C}^{0}-\mathcal{K}$-finitos e ainda podomos assumir que $f_{n-1}^{-1}(0)=g_{n-1}^{-1}(0)$. Daí, pelos resultados obtidos para funções, segue que os germes $f_{n-1}$ e $g_{n-1}$ são $\mathcal{C}^{0}-\mathcal{K}$-equivalentes. Portanto, $f$ e $g$ são $\mathcal{C}^{(0}$ - $\mathcal{K}$-equivalentes.

Caso III. Se f tem coposto 1 e g tem coposto 0.

Podemos assumir

$$
f\left(x_{1}, \ldots, x_{n}\right)=\left(x_{1}, \ldots, x_{n-2}, \int_{n \cdot 1}\left(x_{n-1}, x_{n}\right)\right)
$$

e

$$
g\left(x_{1}, \ldots, x_{n}\right)=\left(x_{1}, \ldots, x_{n-2}, x_{n-1}\right) .
$$

Fazendo o mesmo procedimento do caso anterior para os germes $f_{n-1}\left(x_{n-1}, x_{n}\right)$ e $g_{n-1}\left(x_{n-1}, x_{n}\right)=x_{n-1}$ também concluímos que $f$ e $g$ são $\mathcal{C}^{0}$-K $\mathcal{K}$-equivalentes.

Sem perda de generalidade, se $f, g:\left(\mathbb{R}^{n}, 0\right) \rightarrow\left(\mathbb{R}^{n-1}, 0\right)$ são germes analíticos que definem curvas com o mesmo número de semi-ramos, podemos supor a menos de um homeomorfismo do $\mathbb{R}^{n}$ que $f^{-1}(0)=g^{-1}(0)$. Com isto, segue da Proposição 5.6 que o número de semi-ramos é um invariante completo da $\mathcal{C}^{0}-\mathcal{K}$-equivalência no caso de germes $\mathcal{C}^{0}-\mathcal{K}$-finitos de coposto $\leq 1$. 


\subsection{Famílias de germes de aplicações}

Esta seção contém os resultados principais do Capítulo 5. Utilizamos a $\mathcal{C}^{0}-\mathcal{V}$-equivalência, a condição de (c)-regularidade e condições de fecho integral para obter informações mais precisas sobre a $\mathcal{C}^{0}-\mathcal{K}$-equivalência, para famílias de germes de aplicações.

Quando estudamos uma família de germes de aplicações, uma questão natural ć buscar condiçôes para que a família seja trivial. O estudo da trivialidade topológica de famílias de germes de aplicações e de variedades analíticas é uma das principais direções de investigação da teoria de singularidades atualmente. Para obter resultados sobre a trivialidade topológica, vários autores têm se dedicado ao estudo da Whitney equisingularidade da família (ver, por exemplo, os trabalhos de T. Gaffney, B. Teissier, M.A.S. Ruas, M.J. Saia, entre outros). Una farnília $F:\left(\mathbb{R}^{n} \times \mathbb{R},\{0\} \times \mathbb{R}\right) \longrightarrow\left(\mathbb{R}^{p}, 0\right)$ é Whitney equisingular se existem estratificações de Whitney de $\mathbb{R}^{n} \times \mathbb{R}$ e de $\mathbb{R}^{p}$, com $\{0\} \times \mathbb{R}$ sendo um estrato, tais que $F$ é uma aplicação Thom estratificada que satisfaz a condição $a_{F}$. No caso particular em que $F$ é uma família con singularidade isolada, dizemos que $F^{\prime}$ é Whitney equisingular se o par de estratos $\left(\mathcal{X}=F^{-1}(0)-(\{0\} \times \mathbb{R}), \mathcal{Y}=\{0\} \times \mathbb{R}\right)$ é Whitney regular. Em muitos trabalhos, a Whitney equisingularidade é obtida através de condições sobre o fecho integral de ideais ou módulos.

Definimos a $\mathcal{C}^{0}-\mathcal{K}$-trivialidade do scguinte modo:

Definição 5.7. Dizemos que uma deformação $F:\left(\mathbb{R}^{n} \times \mathbb{R}, 0\right) \longrightarrow\left(\mathbb{R}^{p}, 0\right)$ de um germe $F_{0}=f:\left(\mathbb{R}^{n}, 0\right) \longrightarrow\left(\mathbb{R}^{p}, 0\right)$ é $\mathcal{C}^{0}-\mathcal{K}$-trivial se existem germes de homeomorfismos

$$
h:\left(\mathbb{R}^{n} \times \mathbb{R}, 0\right) \longrightarrow\left(\mathbb{R}^{n} \times \mathbb{R}, 0\right) \text { e } H:\left(\mathbb{R}^{n} \times \mathbb{R}^{p} \times \mathbb{R}, 0\right) \longrightarrow\left(\mathbb{R}^{n} \times \mathbb{R}^{p} \times \mathbb{R}, 0\right),
$$

$h(x, t)=\left(\bar{h}_{t}(x), l\right), \bar{h}_{0}(x)=x, \bar{h}_{t}(0)=0, I I(x, y, t)=\left(\bar{H}_{t}(x, y), t\right), \vec{H}_{0}(x, y)=(x, y)$, $\ddot{H}_{t}(0,0)=(0,0), H\left(\mathbb{R}^{n} \times\{0\} \times\{t\}\right)=\mathbb{R}^{n} \times\{0\} \times\{t\}$ e tais que os seguintes diagramas comutam

$$
\begin{aligned}
& \left(\mathbb{R}^{n} \times \mathbb{R}, 0\right) \stackrel{(i d, f)}{\longrightarrow}\left(\mathbb{R}^{n} \times \mathbb{R}^{p} \times \mathbb{R}, 0\right) \stackrel{\pi_{n+1}}{\longrightarrow}\left(\mathbb{R}^{n} \times \mathbb{R}, 0\right) \\
& h \downarrow \quad H \downarrow \quad h \downarrow \\
& \left(\mathbb{R}^{n} \times \mathbb{R}, 0\right) \stackrel{(i d, F)}{\longrightarrow}\left(\mathbb{R}^{n} \times \mathbb{R}^{p} \times \mathbb{R}, 0\right) \stackrel{\pi_{n+1}}{\longrightarrow}\left(\mathbb{R}^{n} \times \mathbb{R}, 0\right)
\end{aligned}
$$

onde id $:\left(\mathbb{R}^{n} \times \mathbb{R}, 0\right) \rightarrow\left(\mathbb{R}^{n} \times \mathbb{R}, 0\right)$ é a identidade e $\pi_{n+1}:\left(\mathbb{R}^{n} \times \mathbb{R}^{p} \times \mathbb{R}, 0\right) \rightarrow\left(\mathbb{R}^{n} \times \mathbb{R}, 0\right)$ a projeção canônica.

Em outras palavras, $\bar{H}_{t}(x, y)=\left(\bar{h}_{t}(x), \theta_{t}(x, y)\right)$, com $\bar{H}_{t}(x, f(x))=\left(\bar{h}_{t}(x), F_{t}\left(\bar{h}_{t}(x)\right) e\right.$ $\theta_{t}(x, 0)=0$. 
Embora a definição acima seja para qualquer par de dimensões $(n, p)$, nossos resultados são apenas para famílias com $n \geq p$. De modo análogo à definição de $\mathcal{C}^{0}-\mathcal{K}$ trivialidade, podemos definir $\mathcal{C}^{0}-\mathcal{V}$-trivialidade (ou trivialidade do conjunto dos zeros), $\mathcal{C}^{0}-\mathcal{R}$-trivialidade, etc., de acordo com as definiçõos das equivalências: $\mathcal{C}^{0}-\mathcal{V}, \mathcal{C}^{0}-\mathcal{R}, \mathcal{C}^{0}-\mathcal{C}$ c $\mathcal{C}^{0}-\mathcal{A}$.

Como nesta seção estamos tratando apenas de famílias de germes de aplicações, diremos que os germes $f, g:\left(\mathbb{R}^{n}, 0\right) \rightarrow\left(\mathbb{R}^{p}, 0\right)$ são $\mathcal{C}^{0}-\mathcal{K}$-equivalentes em família (ou fortemente $\mathcal{C}^{0}-\mathcal{K}$-equivalentes) se para todo representante $\tilde{f}$ de $f$ e $\tilde{g}$ de $g$, os quais continuaremos denotando por $f$ e $g$, existe uma família $F: U \times[0,1] \rightarrow \mathbb{R}^{p}, U$ uma vizinhança da origem em $\mathbb{R}^{n}$, com $F(x, 0)=f(x), F(x, 1)=g(x)$ c tal que para qualquer $t \in[0,1], F_{t}$ é $\mathcal{C}^{0}-\mathcal{K}$-equivalente a $f$ (resp. $g$ ).

Claro que se a família $F$ é $\mathcal{C}^{0}-\mathcal{K}$-trivial, então $f$ e $g$ são fortemente $\mathcal{C}^{0}-\mathcal{K}$-equivalentes. No caso de funções $(p=1)$, a proposição seguinte mostra que a $\mathcal{C}^{0}-\mathcal{K}$-equivalência e a $\mathcal{C}^{0}-\mathcal{K}$-equivalência forte correspondem à mesma noção.

Proposição 5.8. Sejam $f, g:\left(\mathbb{R}^{n}, 0\right) \rightarrow(\mathbb{R}, 0)$ germes $\mathcal{C}^{0}-\mathcal{K}$-finitos, $n \geq 2$. Suponha que $f \stackrel{\mathcal{C}^{0} \_\mathcal{K}}{\sim} g$. Então, para todo representante $\tilde{f}$ de $f$ e $\tilde{g}$ de $g$, os quais continuaremos denotando por $f$ e $g$, existe um caminho $F: U \times[0,1] \rightarrow \mathbb{R}, U$ uma vizinhança da origem em $\mathbb{R}^{n}$, com $F(x, 0)=f(x), F(x, 1)=g(x)$ e tal que para todo $t \in[0,1], F_{t}$ é $\mathcal{C}^{0}-\mathcal{K}$-equivalente a $f$ (resp. g). Ou seja, $f$ e g são fortemente $\mathcal{C}^{0}-\mathcal{K}$-equivalentes.

Prova. Sejam $f$ e $g$ dois germes de funçẫo cm uma messma $\mathcal{C}^{0}-\mathcal{K}$-órbita, isto é, $f \stackrel{\mathcal{C}}{0} \mathcal{K}^{\sim} g$. Daí, existe um germe de homcomorfismo $h:\left(\mathbb{R}^{n}, 0\right) \rightarrow\left(\mathbb{R}^{n}, 0\right)$ tal que $h\left(f^{-1}(0)\right)=g^{-1}(0)$. Como $f$ (resp. $g$ ) é $\mathcal{C}^{0}-\mathcal{K}$-finito, para qualquer representante $\tilde{f}$ de $f$ (resp. $\tilde{g}$ de $g$ ) existe uma vizinhança $U$ da origem $\mathrm{cm} \mathbb{R}^{n}$ tal que todo ponto de $\tilde{f}^{-1}(0) \cap U-\{0\}$ (resp. $\left.\tilde{g}^{-1}(0) \cap U-\{0\}\right)$ é regular (ver [6̃̃]). Então, como germes na origem, uma das seguintes condições acontecem:

a) sinal $[f(x)]=$ sinal $[g \circ h(x)]$, para todo $x \in U-f^{-1}(0)$, ou

b) sinal $[f(x)]--\operatorname{sinal}[-g \circ h(x)]$, para todo $x \in U-f^{-1}(0)$.

Suponhamos que a) ocorra. Considere a homotopia linear $F_{t}:\left(\mathbb{R}^{n}, 0\right) \rightarrow(\mathbb{R}, 0)$, $t \in[0,1]$,

$$
F_{t}(x)=(1-t) f(x)+t(g \circ h)(x) .
$$

Então, $F_{0}=f, F_{1}=g \circ h, F_{t}^{-1}(0)=f^{-1}(0)$ para todo $t \in[0,1]$ e para quaisquer representantes de $f, g$ e $h$, existe uma vizinhança $U$ da origem em $\mathbb{R}^{n}$ tal que a condição 
iii) do Lema 2.14 está satisfeita. Então, pelo Lema $2.14, F_{t}$ é $\mathcal{C}^{0}-\mathcal{K}$-equivalente a $F_{t^{\prime}}$, quaisquer que sejam $t, t^{\prime} \in[0,1]$. Portanto, $f$ e $g$ são fortemente $\mathcal{C}^{0}-\mathcal{K}$-equivalentes.

Para farnília de germes de aplicações com singularidade isolada, os trabalhos de H.C. King [27] mostram que a trivialidade topológica do conjunto dos zeros implica na $\mathcal{C}^{0}-\mathcal{R}$ trivialidade. Consequentemente, na $\mathcal{C}^{0}-\mathcal{K}$-trivialidade.

Definição 5.9. Seja $n \geq p$. Dizemos que uma família de germes de aplicações polinomiais $F_{t}:\left(\mathbb{R}^{n}, 0\right) \rightarrow\left(\mathbb{R}^{p}, 0\right)$ é uma boa deformação se existe uma vizinhança da origem $U \subset \mathbb{R}^{n}$ tal que, se $x \in U$ com $x \in \Sigma F_{t}$ para algum $t \in \mathbb{R}$, então $x=0$.

Teorema 5.10. (Kin.g [27]) Seja $F_{t}:\left(\mathbb{R}^{n}, 0\right) \rightarrow\left(\mathbb{R}^{p}, 0\right), t \in \mathbb{R}$, uma familia contínua de aplicaşões polinomiais com a condição de boa deformação. Suponha que exista uma família de germes de homeomorfismos $G_{t}:\left(\mathbb{R}^{n}, 0\right) \rightarrow\left(\mathbb{R}^{n}, 0\right)$ tais que $G_{t}\left(F_{t}^{-1}(0)\right)=F_{0}^{-1}(0)$ para todo t. Então, existem uma família de germes de homeomorfismos $H_{t}:\left(\mathbb{R}^{n}, 0\right) \rightarrow\left(\mathbb{R}^{n}, 0\right)$, $t \in \mathbb{R}$, e uma vizinhança $V$ de 0 em $\mathbb{R}$ tais que $F_{t} \circ H_{t}=F_{0}$, para todo $t \in V$.

Adaptando o resultado de King para o nosso contexto do caso $p=n-1$, obtemos o seguinte corolário:

Corolário 5.11. Seja $F_{t}:\left(\mathbb{R}^{n}, 0\right) \rightarrow\left(\mathbb{R}^{n-1}, 0\right), t \in[0,1]$ uma família contínua de aplicações polinomiais com a condição de boa deformação. Suponha que $F_{t}$ defina uma família de germes de curvas $X_{\iota}=F_{t}^{-1}(0)$ com o mesmo número de semi-ramos. Então, $F_{t} \mathcal{e}^{0}{ }^{0}-\mathcal{K}$ equivalente a $F_{0}$, para todo $t \in[0,1]$.

Prova. Por simplicidade, assumiremos que para todo $t \in[0,1], X_{t}=F_{0}^{-1}(0)$, on seja, para todo $t \in[0,1], F_{t}$ define o mesmo germe de curva. Aplicando o Teorema 5.10 para a familia constante $G_{t}=i d$, segue que $F_{t}$ é $\mathcal{C}^{0}-\mathcal{R}$-equivalente a $F_{0}$, para todo $t \in[0,1] \mathrm{c}$, portanto, $\mathcal{C}^{0}-\mathcal{K}$-equivalente.

Assim, para famílias que são "boas deformações", o número de semi-ramos ć um invariante completo para a $\mathcal{C}^{0}-\mathcal{K}$-equivalência.

Neste trabalho, não abordamos o estudo dos germes quase-homogêncos, pois os tipos de resultados que procuramos já existem para esta classe de germes. Por exemplo, os trabalhos de M.A.S. Ruas e M.J. Saia (ver [52], [53]) fornecem estimativas para o grau de $\mathcal{C}^{0}-\mathcal{K}$-determinação e condições para a $\mathcal{C}^{0}-\mathcal{K}$-trivialidade dos germes de aplicações quasehomogêneas em função dos pesos e graus de homogencidade. Estes resultados foram estendidos por C.H. Soares Jr. [58] para uma classe mais geral, obtendo estimativas para 
a. $\mathcal{C}^{0}-\mathcal{K}$-trivialidade em famílias que satisfazem uma condição de não-degeneração com relação a um poliedro de Newton.

Nos resultados que mostraremos a seguir, diremos que $F:\left(\mathbb{R}^{n} \times[0,1], 0\right) \rightarrow\left(\mathbb{R}^{p}, 0\right)$, co uma família fracamente $\mathcal{C}^{0}-\mathcal{K}$-trivial, se a definição topológica de Montaldi é satisfeita para os pares de subvariedades $\left(\operatorname{graf}\left(F_{t}\right), \mathbb{R}^{n} \times\{0\}\right)$, para todo $t \in[0,1]$. Ou seja, existe uma família de homeomorfismos $H:\left(\mathbb{R}^{n} \times \mathbb{R}^{p} \times[0,1], 0\right) \rightarrow\left(\mathbb{R}^{n} \times \mathbb{R}^{p} \times[0,1], 0\right)$ tal que

$$
H_{t}\left(\operatorname{graf}\left(F_{t}\right)\right)=\operatorname{graf}\left(F_{0}\right) \text { e } H_{t}\left(\mathbb{R}^{n} \times\{0\}\right)=\mathbb{R}^{n} \times\{0\},
$$

para todo $t \in[0,1]$, ou nas notações do Capítulo 2,

$$
C^{0}-K\left(\operatorname{graf}\left(F_{t}\right), \mathbb{R}^{n} \times\{0\}, 0\right)=C^{0}-K\left(\operatorname{graf}\left(F_{0}\right), \mathbb{R}^{n} \times\{0\}, 0\right) .
$$

Dada a família a um parâmetro $F:\left(\mathbb{R}^{n} \times \mathbb{R},\{0\} \times \mathbb{R}\right) \rightarrow\left(\mathbb{R}^{p}, 0\right)$ de germes $\mathcal{C}^{0}-\mathcal{K}$-finitos, considere $F_{t}(x)=F(x, t), X_{t}=F_{t}^{-1}(0)-\{0\} \subset\left(\mathbb{R}^{n}, 0\right)$, para todo $t \in \mathbb{R}$,

$$
\mathcal{X}=F^{-1}(0)-(\{0\} \times \mathbb{R}) \subset\left(\mathbb{R}^{n} \times \mathbb{R},\{0\} \times \mathbb{R}\right) \text { e } \mathcal{Y}=\{0\} \times \mathbb{R}
$$

Se o par $(\mathcal{X}, \mathcal{Y})$ acima satisfaz a condição de (c)-regularidade com relação a uma função não-negativa $\rho: \mathbb{R}^{n} \rightarrow \mathbb{R}$ tal que $\rho^{-1}(0)=\mathcal{Y}$ (Definição 1.20), dizemos que a família $X_{t}$, dos conjuntos de zeros de $F_{t}$, é (c)-regular.

Teorema 5.12. Seja $F:\left(\mathbb{R}^{n} \times[0,1],\{0\} \times[0,1]\right) \rightarrow\left(\mathbb{R}^{p}, 0\right), n \geq p$, uma família a um parâmetro de germes $\mathcal{C}^{0}-\mathcal{K}$-finitos. Suponha que a família $X_{t}=F_{t}^{-1}(0)-\{0\}$ seja (c)-regular com relação a uma função $\rho$. Então, $F$ é fracamente $\mathcal{C}^{0}-\mathcal{K}$-trivial.

Prova. Seja $V=\left\{(x, y, t) \in \mathbb{R}^{n} \times \mathbb{R}^{p} \times[0,1] \mid y=0\right.$ ou $\left.y-F(x, t)=0\right\}$ um conjunto semialgébrico em $\mathbb{R}^{n} \times \mathbb{R}^{p} \times[0,1]$.

Chame $J=[0,1]$ e considere a seguinte estratificação $\Xi$ de $V$ :

- $\mathcal{Y}_{0}=\{0\}_{n} \times\{0\}_{p} \times J$;

- $\mathcal{Y}_{1}=\left\{(x, 0, t) \in \mathbb{R}^{n} \times\{0\}_{p} \times J\right\} \cap\left\{F^{-1}(0)-\left(\{0\}_{n} \times J\right)\right\}$;

- $\mathcal{Y}_{21}=\left\{(x, 0, t) \in \mathbb{R}^{n} \times\{0\}_{p} \times J\right\} \cap\left\{\left(\mathbb{R}^{n} \times J\right)-F^{-1}(0)\right\}$;

- $\mathcal{Y}_{22}=\left\{(x, F(x, t), t\} \in \mathbb{R}^{n} \times \mathbb{R}^{p} \times J \mid F(x, t) \neq 0\right\}$.

Afirmação. A estratificação $\Xi$ é (c)-regular com relação a função $\rho$.

De fato, como por hipótese a família $X_{t}$ é (c)-regular, obtemos a (c)-rcgularidade para o par de estratos $\left(\mathcal{Y}_{1}, \mathcal{Y}_{0}\right)$. Para os outros pares de estrato $\left(\mathcal{Y}_{i}, \mathcal{Y}_{j}\right)$ de $\Xi$, a condição 
de (c)-regularidade é trivialmente satisfeita pois $\mathcal{Y}_{j}$ é subvariedade de $\mathcal{Y}_{i}$. Portanto a Afirmaşăo está demonstrada.

O fato do par $\left(\mathcal{Y}_{1}, \mathcal{Y}_{0}\right)$ ser (c)-regular, e portanto a cstratificação $\Xi$ ser (c)-regular, implica que o campo de vetores $\frac{\partial}{\partial t}$ em $\mathcal{Y}$ se levantia a um campo de vetores integrável, tangente aos estratos da estratificação e controlado pelo sistema de tubos $\rho=$ constante. A integrabilidade deste campo implica na existência de uma família de homeomorfismos $H_{t}:\left(\mathbb{R}^{n} \times \mathbb{R}^{p}, 0\right) \rightarrow\left(\mathbb{R}^{n} \times \mathbb{R}^{p}, 0\right)$, preservando estratos, satisfazendo a propriedade $\rho\left(H_{t}(x, y)\right)=\rho(x, y)$ e trivializando a estratificação (para maiores detalhes ver Bekka [3]). Ou seja, existe uma família de homeomorfismos $H_{t}$ do $\mathbb{R}^{n} \times \mathbb{R}^{p}$ que preserva $\mathbb{R}^{n} \times\{0\}$ e leva (graf $\left.\left(F_{t}\right)\right)$ no graf $\left(F_{0}\right)$. Portanto, $F$ é fracamente $\mathcal{C}^{0}-\mathcal{K}$-trivial.

Observe que a $\mathcal{C}^{0}$ - $\mathcal{K}$-trivialidade de $F$ não implica a condição de (c)-regularidade da família $X_{t}=F_{t}^{-1}(0)-\{0\}$. De fato, considere a família $F:\left(\mathbb{R}^{2} \times \mathbb{R}, 0\right) \rightarrow(\mathbb{R}, 0)$

$$
F(x, y, t)=(1-t) x+t y\left(x^{2}+y^{2}\right)
$$

A família $F$ é $\mathcal{C}^{0}-\mathcal{K}$-trivial mas $X_{t}$ não é (c)-regular (ver [54]).

A proposiçâo a seguir é uma aplicação do Teorema 5.12 para germes de classe $C^{r}$.

Proposição 5.13. Considere a família $F:\left(\mathbb{R}^{n} \times(-\varepsilon, \varepsilon),\{0\} \times(-\varepsilon, \varepsilon)\right) \rightarrow\left(\mathbb{R}^{p}, 0\right), n \geq p$, tal que $F(x, t)=f_{t}(x)$, onde $f_{t}:\left(\mathbb{R}^{n} ; 0\right) \rightarrow\left(\mathbb{R}^{p}, 0\right),|t|<\varepsilon$, é uma deformação de uma aplicação $f=f_{0}$ de classe $C^{r}$ com $j^{r} f_{t}(0)=j^{r} f(0)$. Suponha que exista uma constante $\alpha>0$ tal que

$$
\sum_{i_{1} \leq \ldots \leq i_{p}, 1}\left|\frac{D\left(f_{1}, \ldots, f_{p}, \rho\right)}{D\left(x_{i_{1}}, \ldots, x_{i_{p+1}}\right)}(x)\right|^{2}+\sum_{i=1}^{p} f_{i}^{2}(x) \geq c|x|^{2 r}
$$

para $|x|<\alpha$, onde $\rho(x)=x_{1}^{2}+\ldots+x_{p}^{2}$ é uma função controle e a primeira parcela da desigualdade (5.1) representa a soma dos quadrados dos $(p+1)$-menores da matriz jacobiana de $(f, \rho)$. Então, $F$ é fracamente $\mathcal{C}^{0}-\mathcal{K}$-trivial.

Prova. Scjam $V$ e $\Xi$ como no Teorema 5.12.

Afirmação. A estratificação $\Xi$ é (c)-regular.

Do mesmo modo como antes, basta verificar a condição de (c)-regularidade para o par de estratos $\left(\mathcal{Y}_{1}, \mathcal{Y}_{0}\right)$ pois para os outros pares de estratos, a condição de (c)-regularidade é trivialmente satisfeita.

Segue de (5.1) que existe um $\beta>0$ tal que

$$
\sum_{i_{1} \leq \ldots \leq i_{p+1}}\left|\frac{D\left(f_{t, 1}, \ldots, f_{t, p}, \rho\right)}{D\left(x_{i_{1}}, \ldots, x_{i_{p+1}}\right)}(x)\right|^{2}+\sum_{i=1}^{p} f_{t, i}^{2}(x) \geq \frac{c}{2}|x|^{2 r},
$$


para $|x|<\beta$ c $|t|<\varepsilon$. De (5.2),

$$
\left|\operatorname{grad}_{(x, t)} F_{i}\right| \geq \frac{c}{2}|x|^{r-1}
$$

$\operatorname{em}\left\{(x, 0, t) \in \mathbb{R}^{n} \times\{0\}_{p} \times(-\varepsilon, \varepsilon)\right\} \cap\left\{F^{-1}(0)-\left(\{0\}_{n} \times(-\varepsilon, \varepsilon)\right)\right\} \cap\{|x|<\beta\} \quad(1 \leq i \leq p)$

$$
\sum_{i_{1} \leq \ldots \leq i_{p+1}}\left|\frac{D\left(F_{1}, \ldots, F_{t}, \rho\right)}{D\left(x_{i_{1}}, \ldots, x_{i_{p+1}}\right)}(x, t)\right|^{2} \neq 0
$$

$\operatorname{em}\left\{(x, 0, t) \in \mathbb{R}^{n} \times\{0\}_{p} \times(-\varepsilon, \varepsilon)\right\} \cap\left\{F^{-1}(0)-\left(\{0\}_{n} \times(-\varepsilon, \varepsilon)\right)\right\} \cap\{|x|<\beta\} \quad(1 \leq i \leq p)$. Além disso, como $j^{r} f_{t}(0)=j^{r} f(0)$, existem constantes $d, \delta>0$ tais que

$$
\left|\frac{\partial F_{i}}{\partial t}(x, t)\right| \leq d|x|^{r}
$$

para $\{|x|<\delta\}$ e $|t|<\varepsilon$

Então, segue de (5.3) e (5.4) que o par $\left(\mathcal{Y}_{1}, \mathcal{Y}_{0}\right)$ c (a)-regular e por (5.5) temos que a condição $(\mathrm{m})$ está satisfeita. Para maiores detalhes ver $|4|$. Pela Proposição 1.22, o par $\left(\mathcal{Y}_{1}, \mathcal{Y}_{0}\right)$ é (c)-regular. Logo, temos a mesma conclusão dada no 'Teorema 5.12.

Observe que a condição (5.1) é natural no nosso contexto, pois ela está relacionada com a definiçãa de $\mathcal{C}^{(0)}-\mathcal{K}$-determinação finita do germe $f$ (ver Proposiçãa 2.7).

Definição 5.14. Um $r$-jato $z \in J^{r}(n, p)$ é $\mathcal{C}^{0}$ - $\mathcal{V}$-suficiente no conjunto dos germes de classe $C^{s}, \mathcal{E}_{n, p}^{s}=\left\{g \in \mathcal{E}_{n, p} \mid g\right.$ é de classe $\left.C^{s}\right\}, s \geq r$, se quaisquer dois germes $f, g \in \mathcal{E}_{n, p}^{s}$ com $j^{r} f(0)=j^{r} g(0)=z$ sấo $\mathcal{C}^{0}$ - -equivalentes.

Proposição 5.15. Seja $f:\left(\mathbb{R}^{n}, 0\right) \rightarrow\left(\mathbb{R}^{p}, 0\right), n \geq p$, um germe de classe $C^{r}$ tal que $j^{r} f(0)$ é $\mathcal{C}^{0}-\mathcal{V}$-suficiente em $\mathcal{E}_{n, p}^{r}$. Seja $g:\left(\mathbb{R}^{n}, 0\right) \rightarrow\left(\mathbb{R}^{p}, 0\right)$ um germe de classe $C^{r}$ tal que $j^{r} f(0)=j^{r} g(0)$. Então, o graf $(f)$ e o graf $(g)$ têm o mesmo tipo de contato topológico com o $\mathbb{R}^{n} \times\{0\}$ na origem.

Prova. Considere a homotopia linear

$$
F(x, t)=(1-t) f(x)+t g(x), \quad t \in[0,1] .
$$

Seja $\Xi$ a estratificação dada no Teorcma 5.12. Como fizemos antes, é possível mostrar que $\Xi$ é (c)-regular (para detalhes ver 14]). Daí, como nos resultados anteriores, $F$ é fracamente $\mathcal{C}^{0}$ - $\mathcal{K}$-trivial e, portanto, o graf $(f)$ e o graf $(g)$ têm o mesmo tipo de contato topológico com o $\mathbb{R}^{n} \times\{0\}$ na origem.

Os resultados que apresentamos acima estabelecem condições que garantem a $\mathcal{C}^{0}-\mathcal{K}$ trivialidade fraca. Finalizamos esta seçâo com o Teorema 5.20, no qual obtemos uma 
condição suficiente para $\mathcal{C}^{0}-\mathcal{K}$-trivialidade. Isto é feito construindo convenientes campos de vetores integráveis, utilizando uma condição de fecho integral. Este mesmo tipo de argumento aparcee em muitos trabalhos [52], [55], [17], entre outros. O conceito de fecho integral de ideais ou módulos é usado por vários autores para a resolução de problemas de trivialidade de famílias.

Sejam $A_{n}$ o ancl dos germes de funções analíticas e $X \subset \mathbb{R}^{n}$ um conjunto analítico. Definimos $A_{X, x}^{p}$ o módulo formado por p-cópias de

$$
A_{X, x}=A_{n} /\langle\text { funçồes que definem } X \text { numa vizinhança de } x \in X\rangle .
$$

Definição 5.16. Sejam $X$ um conjunto analítico real, e $\mathcal{M}$ um subrnódulo de $A_{X, x}^{p}$. Então, o fecho integral de $\mathcal{M}$ em $A_{X, x}^{p}$, denotado por $\overline{\mathcal{M}}$, é o conjunto dos germes $h \in A_{X, x}^{p}$ tais que para toda curva analitica $\phi:(\mathbb{R}, 0) \rightarrow(X, x)$, temos $九 \circ \phi \in\left(\phi^{*}(\mathcal{M})\right) A_{1}$.

Sejam $\mathcal{M}$ um submódulo de $A_{X, x}^{p},[\mathcal{M}]$ a matriz dos geradores de $\mathcal{M}$, e $J_{k}(\mathcal{M})$ o ideal gerado pelos menores $k \times k$ de $[\mathcal{M}]$.

Lema 5.17. (Gaffney, [22]) Suponha que $h \in A_{X, x}^{p}, \mathcal{M} \subset A_{X, x}^{p}, J_{k+11}((h, \mathcal{M}))=0$. Então,

$$
J_{k}(\mathcal{M}) . h \subseteq \mathcal{M} . J_{k}((h, \mathcal{M}))
$$

A conexão entre fecho integral de ideais e módulos é dada pela seguinte proposição:

Proposição 5.18. (Gaffnèy, [22/) Suponha que $h \in A_{X, x}^{p}, \mathcal{M} \subset A_{X, x}^{p}$ Então, $h \in \overline{\mathcal{M}}$ se, e somente se, $J_{k}((h, \mathcal{M})) \subseteq \overline{J_{k}(\mathcal{M})}$, onde $k$ é o maior inteiro tal que $J_{k}((h, \mathcal{M})) \neq 0$.

No que segue, $\Gamma^{\prime}(E)$ denotará o conjunto das seções do fibrado vetorial $E$.

Proposição 5.19. (Gaffney [220]) Suponha $h \in A_{X, x}^{p}, \mathcal{M} \subset A_{X, x}^{p}$. Então, $h \in \overline{\mathcal{M}}$ se, e somente se, para cada escolha de geradores $\left\{s_{i}\right\}$ de $\mathcal{M}$, existe uma vizinhança $U$ de $x$ lal que pura toda $\phi \in \Gamma\left(\operatorname{Hom}\left(\mathbb{R}^{p}, \mathbb{R}\right)\right)$,

$$
\|\phi(z) \cdot h(z)\| \leq \operatorname{csup}{ }_{i}\left\|\phi(z) \cdot s_{i}(z)\right\|
$$

para todo $z \in U$.

Uma deformação analítica $F:\left(\mathbb{R}^{n} \times \mathbb{R}, 0\right) \rightarrow\left(\mathbb{R}^{p}, 0\right)$ de un germe $F_{0}:\left(\mathbb{R}^{n}, 0\right) \rightarrow\left(\mathbb{R}^{p}, 0\right)$ é chamada boa $\mathcal{K}$-deformação se existe uma vizinhança $U$ da origem em $\mathbb{R}^{n}$ tal que

$$
U \cap F_{t}^{-1}(0) \cap \Sigma F_{t}-\{0\}=\emptyset
$$

para todo $t \in \mathbb{R}$. 
Teorema 5.20. Seja $F:\left(\mathbb{R}^{n} \times \mathbb{R}, 0\right) \rightarrow\left(\mathbb{R}^{p}, 0\right)$ uma boa $\mathcal{K}$-deformação analítica de um germe $F_{0}=f:\left(\mathbb{R}^{n}, 0\right) \rightarrow\left(\mathbb{R}^{p}, 0\right)$. Considere $F_{t}(x)=F(x, t)$. Se

$$
\frac{\partial F}{\partial t} \in \overline{\mathrm{d} F_{t}\left(m_{n} \theta_{n+1}\right)+m_{n} F^{\star}\left(m_{p}\right) \theta_{F_{i}}}
$$

então $F$ é $\mathcal{C}^{0}-\mathcal{K}$-trivial.

Prova. Por simplicidade, denotarmos $h=\frac{\partial F}{\partial t}$ e $\mathcal{M}=\mathrm{d} F_{t}\left(m_{n} \theta_{n+1}\right)+m_{n} F^{\star}\left(m_{p}\right) \theta_{F_{t}}$. Consideremos $\left\{\alpha_{1}, \ldots, \alpha_{m}\right\}$ os geradores de $m_{n} \theta_{n+1}$ onde cada elemento $\alpha_{s}$ é do tipo $x_{i} \frac{\partial}{\partial x_{j}}$, para algum $i=1, \ldots, n$ e algum $j=1, \ldots, n+1$. Sejam $\left\{\mathrm{d} F_{t}\left(\alpha_{1}\right), \ldots, \mathrm{d} F_{t}\left(\alpha_{m}\right)\right\}$ os geradores do submódulo d $F_{t}\left(m_{n} \theta_{n+1}\right)$ e $\left\{x_{l} F_{t, i} e_{j}, l=1, \ldots, n ; i=1, \ldots p ; j=1, \ldots, p\right\}$ os geradores do submódulo $m_{n} F^{\star}\left(m_{p}\right) \theta_{F_{t}}$.

Sejam $\left\{\rho_{1}, \ldots, \rho_{r}\right\}$ os geradores de $J_{p}(\mathcal{M})$ e $\rho=\sum_{i=1}^{r} \rho_{i}^{2}$.

Como por hipótese, $F$ é uma boa $\mathcal{K}$-deformação analítica, então $\rho(x)=0 \Leftrightarrow x=0$. Observe que

$$
\rho_{k} \cdot h \in J_{p}(\mathcal{M}) \cdot h
$$

Então, pelo Lema 5.17,

$$
\rho_{k} \cdot h \in \mathcal{M} \cdot J_{p}((h, \mathcal{M}))
$$

Portanto, podemos escrever

$$
\rho_{k} \cdot h=\sum_{j}^{m} \mathrm{~d} F_{t}\left(\alpha_{j}\right) a_{k j}+\sum_{l, i, j}^{n, p, p} x_{l} F_{t, i} e_{j} b_{i j k},
$$

$\operatorname{com} a_{k j}, b_{i j k} \in J_{p}((h, \mathcal{M}))$.

Como $\rho_{k}$ é um gerador de $J_{p}(\mathcal{M})$, podemos assumir que $\rho_{k}$ é um menor $p \times p$ da matri\% $[\mathcal{M}$ ]. De acordo com as notações usadas no Capítulo 2 (Proposição 2.7) e na expressão (5.1), podemos assumir, que os $\rho_{k}^{2}$ são exatamente os elementos que aparecem em

$$
N_{C} F_{t}(x)=\sum_{i=1}^{p} F_{t, i}^{2}(x) \text { ou } N_{\mathcal{R}} F_{t}(x)=\sum_{i_{1} \leq \ldots \leq i_{p+1}}\left|\frac{D\left(F_{l, 1}, \ldots, F_{t, p}\right)}{D\left(x_{i_{1}}, \ldots, x_{i_{p+1}}\right)}(x)\right|^{2} .
$$

Assim,

$$
\rho=\sum_{i=1}^{r} \rho_{i}^{2}=N_{\mathcal{C}} F_{t}(x)+N_{\mathcal{R}} F_{t}(x)=N_{\mathcal{K}} F_{t}(x) .
$$

Segue dos resultados de Gaffney [21] que podemos explicitar os elementos que aparecem em (5.7). Por exemplo, se $\rho_{k}$ é o determinante de um menor $p \times p M$ da matriz jacobiana (com respeito a $x$ ) de $\mathrm{d} F_{t}$, podemos escrever

$$
(\operatorname{det} M)\left[\begin{array}{c}
h_{1} \\
\vdots \\
h_{p}
\end{array}\right]=\mathrm{d} F_{t}\left[\sum_{s=1}^{p} \sum_{r=1}^{p}\left[\operatorname{cof}\left(\frac{\partial\left(F_{t}\right)_{r}}{\partial x_{i_{s}}}\right) h_{r}\right] \frac{\partial}{\partial x_{i_{s}}}\right],
$$


onde cof representa os cofatores da matriz. Seja $\hat{M}=\operatorname{cof}\left(M^{t}\right)$.

De (5.8) segue que

$$
M . \hat{M}\left[\begin{array}{c}
h_{1} \\
\vdots \\
h_{p}
\end{array}\right]=\mathrm{d} F_{t}\left[\begin{array}{c}
0 \\
\vdots \\
0 \\
\operatorname{cof}\left(\frac{\partial F_{t, 1}}{\partial x_{i_{1}}}\right) h_{1}+\ldots+\operatorname{cof}\left(\frac{\partial F_{t, p}}{\partial x_{i_{1}}}\right) h_{p} \\
\operatorname{cof}\left(\frac{\partial F_{t, 1}}{\partial x_{i_{p}}}\right) h_{1}+\ldots+\operatorname{cof}\left(\frac{\partial F_{t, p}}{\partial x_{i_{p}}}\right) h_{p} \\
0 \\
\vdots \\
0
\end{array}\right] .
$$

As linhas de índices $k$, com $k \neq i_{s}$ são nulas.

Denotamos por

$$
{ }^{\star} M_{t}^{J} h=\sum_{s=1}^{p} \sum_{r=1}^{p}\left[\operatorname{cof}\left(\frac{\partial\left(F_{t}\right)_{r}}{\partial x_{i_{s}}}\right) h_{r}\right] \frac{\partial}{\partial x_{i_{s}}},
$$

onde $J$ enumera os possiveis $p \times p$-menores de $\mathrm{d} F_{l}$.

Então,

$$
N_{\mathcal{R}} F_{t} \cdot h=\sum_{j=1}^{l}\left[\mathrm{~d} F_{t}\left({ }^{*} M_{t}^{J}\left(\operatorname{det} M_{t}^{J}\right) h\right] .\right.
$$

Do mesmo modo, se consideramos $\rho_{k} \cdot h$ em (5.7), com $\rho_{k}^{2}$ igual a uma parcela da soma $N_{\mathcal{C}} F_{t}$, podemos explicitar a cxpressão $\rho_{k}^{2} \cdot h$ e concluir que

$$
N_{\mathcal{C}} F_{t} \cdot h=\sum_{i=1}^{p}\left[F_{t, i} \cdot h\right] F^{\star}\left(y_{i}\right) .
$$

Estes cálculos são feitos com detalhes por Ruas em [51]

Voltando à expressão (5.7) temos que

$$
\rho \cdot h=\mathrm{d} F_{t}\left[\sum_{k, j}^{r, m} a_{k j} \rho_{k} \alpha_{j}\right]+\sum_{l, i, j, k}^{n, p, p, r} \rho_{k} b_{i j k} x_{l} e_{j} F_{t, i} .
$$

Pela hipótese $h \in \overline{\mathcal{M}}$, e pela Proposição 5.18 , temos que

$$
J_{p}((h, \mathcal{M})) \subseteq \overline{J_{p}(\overline{\mathcal{M}})}
$$

Então, segue que $a_{k j}, b_{i j k} \in \overline{J_{p}(\mathcal{M})}$.

Considere o campo $\varepsilon(x, t)=\sum_{k, j}^{r, m} \frac{a_{k j} \rho_{k} \alpha_{j}}{\rho}$. 
Usando a versão da Proposição 5.19 para ideais (ver [22]), temos que se $a_{k j} \in \overline{J_{p}(\mathcal{M})}$ então

$$
\begin{gathered}
\left|a_{k j}\right| \leq c_{1} \sup _{i}\left\{\text { geradores de } J_{p}(\mathcal{M})\right\}, \text { ou seja, } \\
\left.\left|a_{k j}\right| \leq c_{1} \sup _{i}\left\{\mid \rho_{i}\right\}\right\}
\end{gathered}
$$

$\Lambda$ ssim, $|\varepsilon(x, t)| \leq c|x|$, o que implica que o campo $\varepsilon$ é contínuo e integrável pois satisfaz uma condição Lipschitz ao longo da solução $0 \times \mathbb{R}$ (ver Kuo [28]).

Seja

$$
\eta(x, y, t)=\sum_{l, i, j, k}^{n, p, p, r} \frac{\left(\rho_{k} b_{i j k} x_{l} e_{j}\right) y_{i}}{\rho} .
$$

Como $b_{i j k} \in \overline{J_{p}(\mathcal{M})}$, novamente temos que

$$
\left|b_{i j k}\right| \leq c_{2} \sup _{i}\left\{\left|\rho_{i}\right|\right\}
$$

e, portanto, $|\eta(x, y, t)| \leq c|x||y|$, o que implica que $\eta$ é integrável.

Quando escrevemos os elementos de forma explicita, como nas expressões (5.10) e (5.11), basta observar que os elementos $a_{k j}, b_{i j k} \in \overline{J_{p}(\mathcal{M})}$ neste caso são dados por

$$
a_{k j}={ }^{\star} M_{t}^{J} h \text { e } b_{i j k}=F_{i, t} .
$$

Com isso podemos escrever as expressões explícitas dos campos $\varepsilon$ e $\eta$ e verificar que eles são integráveis, como fizemos anteriormentc.

Como $\varepsilon$ e $\eta$ são integráveis, o fluxo gerado por eles fornece os homeomorfismos que estabelecem a $\mathcal{C}^{0}-\mathcal{K}$-trivialidade da farnília $F$.

Seria interessante obter um resultado análogo ao Teorema 5.20 mas com a hipótese

$$
\frac{\partial F}{\partial t} \in \overline{\mathrm{d} F_{t}\left(m_{n} \theta_{n+1}\right)+F^{\star}\left(m_{p}\right) \theta_{F_{t}}}
$$

Pretendemos dar continuidade a este estudo.

Teorema 5.21. (Gaffney [22]) Seja $F:\left(\mathbb{R}^{n} \times \mathbb{R}, 0\right) \rightarrow\left(\mathbb{R}^{p}, 0\right)$ uma familia analitica tal que

$$
\frac{\partial F}{\partial t} \in \overline{\left\{x_{i} \frac{\partial F_{t}}{\partial x_{j}}\right\}_{\mathcal{A}_{X}^{p}} .}
$$

Então, o par de estratos $\left(\mathcal{X}=F^{-1}(0)-(\{0\} \times \mathbb{R}), \mathcal{Y}=\{0\} \times \mathbb{R}\right)$ é Whitney regular.

Se o par $(\mathcal{X}, \mathcal{Y})$ acima é Whitney regular, então $(\mathcal{X}, \mathcal{Y})$ é (c)-regular (ver [3]). Daí, pclo Teorema 5.12 , a família $F$ é fracamente $\mathcal{C}^{0}-\mathcal{K}$-trivial. Ou scja, obtemos o corolário: 
Corolário 5.22. Seja $F:\left(\mathbb{R}^{n} \times \mathbb{R}, 0\right) \rightarrow\left(\mathbb{R}^{p}, 0\right)$ uma família analítica de germes $\mathcal{C}^{0}-\mathcal{K}$ finilos. Se

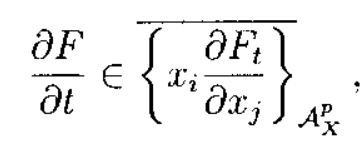

então, $F$ é fracamente $\mathcal{C}^{0}-\mathcal{K}$-trivial.

O Teorema 5.20 é um análogo para o caso real de um resultado geral obtido por J.N Tomazella [61]. No caso complexo, vale a volta do Teorema 5.21 . 


\section{Capítulo 6}

\section{Equivalência de contato bi-Lipschitz (K-bi-Lipschitz equivalência)}

Como é conhecido em teoria de singularidades, a classificação de germes de aplicações módulo difcomorfismos apresenta muita rigidez. Observe, por exemplo, a família

$$
F_{t}(x, y)=x y(x-y)(x-t y), \quad 0<t<1
$$

introduzida por Whitney (1965). Neste caso, para quaisquer $t \neq t^{\prime} \in(0,1)$, não ć possivel construir um difeomorfismo $H:\left(\mathbb{R}^{2}, 0\right) \rightarrow\left(\mathbb{R}^{2}, 0\right)$, de classe $C^{1}$, tal que $F_{t}=F_{t^{\prime}} \circ H$

Por outro lado, a classificação topológica pode ser muito flexivel (ver Teorema 3.6). Lntão, é natural pensar em classificações de germes obtidas por meio de relações de equivalências mais fracas do que diferenciáveis e mais fortes do que topológicas. Isso motiva, por exemplo, o estudo da equivalência bi-Lipschitz dos germes de aplicações. Muitos autores têm estudado as propriedades e invariantes desta relação de equivalência ([41], [50], [7], [17], [25] entre outros).

Seja $c \in \mathbb{R}$ um número real positivo. Lma aplicação $I: U \subset \mathbb{R}^{n} \rightarrow \mathbb{R}^{p}$ é chamada c-Lipschitz, ou simplesmente Lipschitz, se satisfaz:

$$
\|H(x)-H(y)\| \leq c\|x-y\|, \quad \forall x, y \in U .
$$

Quando $n=p$ e $H$ admite inversa Lipschitz, dizemos que $H$ é bi-Lipschitz. Ilomeomorfismos bi-Lipschitz são interessantes por pelo menos duas razões:

1. Eles têm boas propriedades que são de interesse geral. Por exemplo, preservam conjuntos de medida nula, o grupo de todos os homeomorfismos bi-Lipschitz tem uma topologia natural, etc... 
2. Eles têm interessantes propriedades métricas. Por exemplo, a propriedade de Lojasiewicz: se $X$ e $Y$ são conjuntos analíticos então para algum $c>0$ e $k>0$ temos, localmente,

$$
\operatorname{dist}(x, Y) \geq k \operatorname{dist}(x, X \cap Y)^{c},
$$

para $x \in X$, onde dist é uma função distância. $O$ melhor expoente $c=c(X, Y)$ é um interessante invariante métrico do par $(X, Y)$.

Vale obscrvar que essas propriedades são preservadas por homeomorfismos bi-Lipschitz mas não necessariamente por homeomorfismos arbitrários. O artigo de T. Mostowski [41] fornece um método para construir homeomorfismos bi-Lipschitz (via campos de vetores) cntre germes de conjuntos analíticos.

Dois germes $f, g:\left(\mathbb{R}^{n}, 0\right) \rightarrow\left(\mathbb{R}^{p}, 0\right)$ são chamados bi-Lipschitz equivalentes se existe um germe de aplicação bi-Lipschitz $H:\left(\mathbb{R}^{n}, 0\right) \rightarrow\left(\mathbb{R}^{n}, 0\right)$ tal que $f=g \circ H$. Esta equivalência é também conhecida como $\mathcal{R}$-bi-Lipschitz equivalência, pois o germe dc homeomorfismo bi-Lipschitz $H$ atua à direita.

J.-P. Henry e A. Parusinski [25] mostraram que a equivalência bi-Lipschitz dos germes de funções analíticas tem moduli, isto é, existem infinitas classes de bi-Lipschitz equivalência para estes germes.

Exemplo 6.1. Considere a família a 1-parâmetro $F_{t}:\left(\mathbb{R}^{2}, 0\right) \longrightarrow(\mathbb{R}, 0)$ dada por $F_{t}(x, y)=x^{3}-3 t^{2} x y^{4}+y^{6}, t \in \mathbb{R}$. Então, para quaisquer $t \neq t^{\prime}, t, t^{\prime}>0$ temos que $F_{t}$ não é bi-Lipschitz equivalente a $F_{t^{\prime}}$, ou seja, não existe um germe de homeomorfismo bi-Lipschitz $H:\left(\mathbb{R}^{2}, 0\right) \longrightarrow\left(\mathbb{R}^{2}, 0\right)$ tal que $F_{t}=F_{t^{\prime}} \circ H$.

Em particular, isto mostra que a equivalência bi-Lipschitz dos germes de funções analíticas reais admite moduli contínuo.

Por outro lado, os tipos bi-Lipschitz dos germes de variedades reais analíticas não têm moduli ([41], [48], [47]). Isso é consequência do seguinte teorema:

Teorema 6.2. (Mostowski [41]) Dado c $>0$, considere o conjunto de todos os conjuntos semialgébricos com complexidade $\leq c$. Fntão, o conjunto dos tipos Lipschitz é finito.

O conceito de complexidade que aparece no enunciado do teorema acima pode ser interpretado na tcoria semialgébrica como sendo a seguinte soma:

número de variávcis + número de desigualdades - grau, 
dos polinômios que definem os conjuntos semialgébricos em questão (para maiores detalhes ver $[41 \mid)$.

A teoria dos invariantes da equivalência bi-Lispchitz está sendo recentemente desenvolvida. Por exemplo, o teorcma a seguir mostra que a multiplicidade é um invarianto desta rclação de equivalência.

Teorema 6.3. (Fernandes-Ruas (17)) Sejam $f, g:\left(\mathbb{R}^{n}, 0\right) \rightarrow(\mathbb{R}, 0)$ germes de funções analíticas. Se $f$ e g são bi-Lipschitz equivalentes, entäo $f$ e $g$ têm a mesma multiplicidade.

Considerando um germe de função analítica $f:\left(\mathbb{R}^{n}, 0\right) \rightarrow(\mathbb{R}, 0)$,

$$
f(x)=f_{m}(x)+f_{m+1}(x)+\ldots,
$$

onde $f_{i}$ é uma forma homogênea de grau $i$ e $f_{m} \neq 0$, recordamos que a multiplicidade de $f, m_{f}$, é definida por $m_{f}:=m$.

Henry e Parusinski [26] definem um invariante bi-Lipschitz que varia continuamento em muitas famílias de germes de funções analíticas. A prova é similar a do caso complexo feita por eles em [25], embora mais delicada. Para um germe $f$ o invariante é dado $\mathrm{cm}$ termos dos coeficientes líderes das expansões assintóticas de $f$ ao longo de certos conjuntos onde é possível comparar $|x||\operatorname{grad} f(x)| \operatorname{com}|f(x)|$.

Ao analisar os resultados de [25] e [41], propomos neste capítulo introduzir uma relação de equivalência mais ampla do que a equivalência bi-Lipschitz: a saber, a equivalência de contato bi-Lipschitz ou $\mathcal{K}$-bi-Lipschitz equivalência. Os principais resultados deste capítulo encontram-se em [9].

Uma motivação para estudar as aplicações bi-Lipschitz está relacionada com uma importante questão em aberto na teoria clássica de singularidades: o problema da estabilidade bi-Lipschitz. As idéias de Whitney (1960) de aproximar aplicações diferenciávcis por aplicações com propriedades especialmente simples, como mergulhos e imersões, deranı origem à noção de estabilidade. Mather, por volta de 1970, determinou os pares de dimensões $(n, p)$ para os quais o conjunto das aplicações $C^{\infty}$-estáveis é denso no conjunto $C_{p r}^{\infty}\left(\mathbb{R}^{n}, \mathbb{R}^{p}\right)=\left\{f:\left(\mathbb{R}^{n}, 0\right) \rightarrow\left(\mathbb{R}^{p}, 0\right) \mid f\right.$ é própria $\}$ com a topologia de Whitney. Estes valores dos pares $(n, p)$ são conhecidos como boas dimensões de Mather. Para aplicações de classe $C^{1}$, Mather mostrou que a densidade não ocorre, ou seja, o conjunto das aplicações $C^{1}$-estáveis não é denso cm $C_{p r}^{\infty}\left(\mathbb{R}^{n}, \mathbb{R}^{p}\right)$ para $(n, p)$ fora das boas dimensões. No caso topológico, ao contrário, a $\mathcal{C}^{0}$-estabilidade é densa em $C_{p r}^{\infty}\left(\mathbb{R}^{n}, \mathbb{R}^{p}\right)$, fora das boas dimensões. Então, uma pergunta natural é saber se no complementar das boas dimensõcs de Mather, as aplicações bi-Lipschitz estáveis são densas em $C_{p r}^{\infty}\left(\mathbb{R}^{n}, \mathbb{R}^{p}\right)$. Além disso, 
sabemos pelos Teoremas 1.43 e 1.44 , que a $\mathcal{K}$-equivalência foi introduzida por Mather para reduzir o problema da classificação das aplicações $C^{\infty}$-estávcis para o problema da classificação de $\mathbb{R}$-álgebras isomorfas. É uma questão aberta se esta mesma abordagem se aplica ao caso bi-Lipschitz, o que motiva introduzir o estudo da $\mathcal{K}$-bi-Lipschitz equivalência dos germes de aplicações diferenciáveis.

Definição 6.4. Dois germes $f, g:\left(\mathbb{R}^{n}, 0\right) \longrightarrow\left(\mathbb{R}^{p}, 0\right)$ são chamados contato bi-Lipschitz equivalentes (ou simplesmente $\mathcal{K}$-bi-Lipschitz equivalentes) se existem germes de homeomorfismos bi-Lipschitz $h:\left(\mathbb{R}^{n}, 0\right) \longrightarrow\left(\mathbb{R}^{n}, 0\right)$ e $H:\left(\mathbb{R}^{n} \times \mathbb{R}^{p}, 0\right) \longrightarrow\left(\mathbb{R}^{n} \times \mathbb{R}^{p}, 0\right)$ tais que $H\left(\mathbb{R}^{n} \times\{0\}\right)=\mathbb{R}^{n} \times\{0\}$ e os seguintes diagramas comutam

$$
\begin{aligned}
& \left(\mathbb{R}^{n}, 0\right) \stackrel{(i d, f)}{\longrightarrow}\left(\mathbb{R}^{n} \times \mathbb{R}^{p}, 0\right) \stackrel{\pi_{n}}{\longrightarrow}\left(\mathbb{R}^{n}, 0\right) \\
& h \downarrow \quad I \downarrow \downarrow h \downarrow \\
& \left(\mathbb{R}^{n}, 0\right) \stackrel{(i d, g)}{\longrightarrow}\left(\mathbb{R}^{n} \times \mathbb{R}^{p} ; 0\right) \stackrel{\pi_{n}}{\longrightarrow}\left(\mathbb{R}^{n} ; 0\right)
\end{aligned}
$$

onde id $:\left(\mathbb{R}^{n}, 0\right) \longrightarrow\left(\mathbb{R}^{n}, 0\right)$ é a aplicação identidade do $\mathbb{R}^{n}$ e $\pi_{n}:\left(\mathbb{R}^{n} \times \mathbb{R}^{p}, 0\right) \longrightarrow\left(\mathbb{R}^{n}, 0\right)$ a projeçâo canônica.

Em outrus palavrus, $H$ é dudo por $H(x, y)=(h(x), \theta(x, y)), \operatorname{com} \theta(x, 0)=0.0$ homeomorfismo bi-Lipschitz $H$ aplica o gráfico de $f$ no gráfico de g, enquanto h atua em $\mathbb{R}^{n}$.

Em particular, definimos a $\mathcal{C}$-bi-Lipschitz equivalência quando $h=i d$.

$\Lambda$ Definiçũo 6.4 á a versão bi-Lipschitz da $\mathcal{K}$-equivalência de Mather.

Exemplo 6.5. Os germes $f(x, y)=x y(x-y)\left(x-\frac{y}{2}\right)$ e $g(x, y)=x y(x-y)\left(x-\frac{y}{5}\right)$ são $\mathcal{K}$-bi-Lipschitz equivalentes. Na verdade estes germes são bi-Lipschitz equivalentes ([17]).

Exemplo 6.6. Ao contrário do que acontece no Exemplo 6.1, existem infinitos valores do parâmelro $t$ para os quais $F_{t}(x, y)=x^{3}-3 t^{2} x y^{4}+y^{6}$ estão em uma mesma $\mathcal{K}$-bi-Lipschitz órbita. (Isto é consequência do Teorema 6.14 que provaremos na Seção 6.1).

Considerando o problema de classificação de germes com rolação a $\mathcal{K}$-bi-Lipschitz equivalência, precisamos saber se este problema é tratável ou não. Por isso, da mesma forma como citado nos Capítulos 2 e 4, queremos entender se o número de classes da $\mathcal{K}$-bi-Lipschitz equivalência é finito. Responderemos isto no Teorema 6.14 para germes de funções polinomiais.

A partir de agora nos restringiremos ao estudo da $\mathcal{K}$-bi-Lipschitz equivalência dos germes de funçoos. 
Definição 6.7. Dadas as funções $f, g: \mathbb{R}^{n} \rightarrow \mathbb{R}$, denotamos $f \lesssim g$ quando existe uma vizinhança $U$ da origem em $\mathbb{R}^{n}$ e uma constante real positiva c tal que $f(x) \leq c g(x)$, para todo $x \in U$. Denotamos $f \approx g$ quando ocorre $f \lesssim g$ e $g \lesssim f$. Em outras palavras, $f \approx g$ se existem constantes reais positivas $c_{1}, c_{2}$ tais que

$$
c_{1} g(x) \leq f(x) \leq c_{2} g(x), \quad \forall x \in U,
$$

$U$ uma vizinhança da origem em $\mathbb{R}^{n}$. Chamaremos $\approx$ de ordern de contato na origem.

Segue da definição acima que se dois germes $f$ e $g$ têm a mesma ordem de contato na origem $(f \approx g)$ então os germes dos conjuntos de zcros são iguais, isto é, $f^{-1}(0)=g^{-1}(0)$ em uma vizinhança $U$ da origem.

Decorre dos trabalhos de B. Teissier [60] e Gaffney |22|, que a definição $f \approx g$, para germes reais analíticos, implica que $\overline{\langle f\rangle_{\mathbb{R}}}=\overline{\langle g}_{\mathbb{R}}$, onde $\overline{\langle f\rangle_{\mathbb{R}}}$ c o fecho integral real de $f$. Lembramos que o fecho integral real de um ideal $I, \bar{I}_{\mathbb{R}}$, ć o conjunto $\operatorname{dos} h \in A_{n}$ tais que para toda curva analítica real $\gamma:(\mathbb{R}, 0) \rightarrow\left(\mathbb{R}^{n}, 0\right), h \circ \gamma \in \gamma^{\star}(I)$, onde $\gamma^{\star} \dot{0}$ a aplicação induzida dada por $\gamma^{\star}(u)=u \circ \gamma$, para todo $u \in I$.

Exemplo 6.8. Os germes $f(x, y)=x^{2}+y^{4}$ e $g(x, y)=x^{2}+y^{2}$ não tem a mesma ordem de contato na origem, embora $f^{-1}(0)=g^{-1}(0)=\{0\}$.

Teorema 6.9. Sejam $f, g:\left(\mathbb{R}^{n}, 0\right) \rightarrow(\mathbb{R}, 0)$ germes de funções Lipschitz. Então, $f$ e $g$ são $\mathcal{C}$-bi-Lipschitz equivalentes se, e somente se, uma das seguintes condiçóes acontece:

i) $f \approx g$

ii) $f \approx-g$.

Prova. Suponha que os germes de funções Lipschitz $f$ e $g$ sejam $\mathcal{C}$-bi-Lipschitz equivalentes. Considere então $H:\left(\mathbb{R}^{n} \times \mathbb{R}, 0\right) \rightarrow\left(\mathbb{R}^{n} \times \mathbb{R}, 0\right)$ um germe de homcomorfismo bi-Lipschitz tal que $I(x, 0)=(x, 0)$ e $H(x, f(x))=(x, g(x))$.

Sejam

$$
V_{+}=\left\{(x, y) \in\left(\mathbb{R}^{n} \times \mathbb{R}, 0\right) \mid y>0\right\} \text { e } V_{-}=\left\{(x, y) \in\left(\mathbb{R}^{n} \times \mathbb{R}, 0\right) \mid y<0\right\}
$$

Afirmação 1. Uma das seguintes condições acontece:

1) $I I\left(V_{+}\right)=V_{+} \quad$ e $\quad H\left(V_{-}\right)=V_{-}$, ou

2) $H\left(V_{+}\right)=V_{-}$e $H\left(V_{-}\right)=V_{+}$ 
De fato, suponha, por absurdo, que existam pontos $a, b, a^{\prime}$ e $b^{\prime}$ tais que $a \in V_{+}, b \in V_{.}$, $H(a)=a^{\prime} \in V_{+}$e $H(b)=b^{\prime} \in V_{+}$. Daí, considere um caminho em $V_{+}$ligando $a^{\prime}$ e $b^{\prime}$. 'Tomando a imagem inversa deste caminho, obtemos um outro caminho ligando $a$ e $b$ que passa por $\mathbb{R}^{n} \times\{0\}$. Mas isto é um absurdo, pois $H(x, 0)=(x, 0)$. Assim, a Afirmação 1 está demonstrada.

Suponha que 1) ocorra. Neste caso, as funçôes $f$ e $g$ têm o mesmo sinal em cada componente conexa do conjunto $f(x) \neq 0$. Além disso,

$|g(x)|=\|(x, 0)-(x, g(x))\|=\|H(x, 0)-H(x, f(x))\| \leq c_{2}\|(x, 0)-(x, f(x))\|=c_{2}|f(x)|$, onde $c_{2}$ é um número real positivo. Procedendo de modo análogo com a inversa de $H$, podemos mostrar também que existe $c_{1}>0$ tal que

$$
c_{1}|f(x)| \leq|g(x)|
$$

e, portanto, $f \approx g$.

Suponha que 2) ocorra, isto é, $H\left(V_{+}\right)=V_{-}$e $H\left(V_{-}\right)=V_{+}$.

Considere $\xi:\left(\mathbb{R}^{n} \times \mathbb{R}, 0\right) \rightarrow\left(\mathbb{R}^{n} \times \mathbb{R}, 0\right)$ um germe de aplicação definido por:

$$
\xi(x, y)=(x,-y)
$$

Aplicando os mesmos argumentos anteriores para $\xi \circ H$, podemos concluir que $f \approx-g$. Reciprocamente, suponha que $f \approx g$ (para $f \approx-g$ a prova ć análoga). Vamos construir um germe de aplicação

$$
\begin{aligned}
H:\left(\mathbb{R}^{n} \times \mathbb{R}, 0\right) & \rightarrow\left(\mathbb{R}^{n} \times \mathbb{R}, 0\right) \\
(x, y) & \mapsto(x, \theta(x, y))
\end{aligned}
$$

do seguinte modo:

$$
H(x, y)= \begin{cases}(x, 0) & \text { se } y=0 \\ \left(x, \frac{g(x)}{f(x)} y\right) & \text { se } 0 \leq|y| \leq|f(x)| \\ (x, y-f(x)+g(x)) & \text { se } 0 \leq|f(x)| \leq|y| \\ (x, y) & \text { nos demais pontos. }\end{cases}
$$

Afirmação 2. H é um germe de aplicação bi-Lipschitz.

De fato, $H$ é injetiva pois, para qualquer $x^{\star}$ fixado, temos que $\theta\left(x^{\star}, y\right)$ é uma função contínua e monótona. Claramente $H$ é Lipschitz se $0 \leq|f(x)| \leq|y|$. Mostremos que $H$ 
é Lipschitz se $0 \leq|y| \leq|f(x)|$. Para isto, é suficiente mostrar que todas as derivadas parciais $\frac{\partial \theta}{\partial x_{i}}$ são limitadas neste domínio, para todo $i=1, \ldots, n$. Com efeito,

$$
\frac{\partial \theta}{\partial x_{i}}=\frac{\left(\frac{\partial g}{\partial x_{i}} f(x)-\frac{\partial f}{\partial x_{i}} g(x)\right) y}{(f(x))^{2}}=\frac{\partial g}{\partial x_{i}} \frac{y}{f(x)}-\frac{\partial f}{\partial x_{i}} \frac{g(x)}{f(x)} \frac{y}{f(x)}
$$

Como $|y| \leq|f(x)|$, então $\frac{y}{f(x)}$ é limitada. A expressão $\frac{g(x)}{f(x)}$ ó limitada pois estamos supondo que $f \approx g$. Mais ainda, $\frac{\partial g}{\partial x_{i}} \mathrm{e} \frac{\partial f}{\partial x_{i}}$ são limitadas pois $f$ e $g$ são funções Lipschit $z$.

Como $H^{-1}$ pode ser construída da mesma forma como (6.1), podemos concluir que $I^{-1}$ ć também Lipschitz e, portanto, $H$ é um germe de aplicação bi-Lipschitz e a Afirmação 2 está demonstrada.

Portanto, como $H(x, y)=(x, \theta(x, y))$ é um germe de homeomorfismo bi-Lipschitz tal que $H(x, 0)=(x, 0)$ e $H(x, f(x))=(x, g(x))$, temos que $f$ e $g$ são $\mathcal{C}$-bi-Lipschitz equivalentes.

Definição 6.10. Seja $h:\left(\mathbb{R}^{n}, 0\right) \rightarrow\left(\mathbb{R}^{n}, 0\right)$ um germe de homeomorfismo bi-Lipschitz. Definimos $f \stackrel{h}{\approx} g$ quando $f \approx g \circ h$.

Corolário 6.11. Sejam $f, g:\left(\mathbb{R}^{n}, 0\right) \rightarrow(\mathbb{R}, 0)$ germes de funções Lipschitz. Então, $f$ e $g$ são $\mathcal{K}$-bi-Lipschitz equivalentes se, e somente se, uma das seguintes condições acontece:

i) $f \stackrel{h}{\approx} g$,

ii) $f \stackrel{h}{\approx}-g$.

O Corolário acima mostra que a condição de ordem de contato na origem $\stackrel{h}{\approx}$ é um invariante completo para a $\mathcal{K}$-bi-Lipschitz equivalência.

Definição 6.12. Dois germes de funções Lipschitz são chamados $\mathcal{K}$-M-bi-Lipschitz equivalentes (ou equivalentes por contato no sentido de Mather-Montaldi) se existe um germe de aplicação bi-Lipschitz $M:\left(\mathbb{R}^{n} \times \mathbb{R}, 0\right) \rightarrow\left(\mathbb{R}^{n} \times \mathbb{R}, 0\right)$ tal que $M\left(\mathbb{R}^{n} \times\{0\}\right)=\mathbb{R}^{n} \times\{0\}$ e $M(\operatorname{graf}(f))=\operatorname{graf}(g)$. A aplicação $M$ é chamada de aplicação de Mather-Montaldi.

O teorema a seguir caracteriza a $\mathcal{K}$-bi-Lipschitz equivalchncia através das idéias geométricas de Mather-Montaldi.

Teorema 6.13. Dois germes de funções Lipschitz $f, g:\left(\mathbb{R}^{n}, 0\right) \rightarrow(\mathbb{R}, 0)$ são $\mathcal{K}$ - $\mathcal{M}$-biLipschitz equivalentes se, e somente se, eles são $\mathcal{K}$-bi-Lipschitz equivalentes. 
Prova. É claro que $\mathcal{K}$-bi-Lipschitz equivalência implica $\mathcal{K}$-M-bi-Lipschitz equivalência.

Sejam $f$ e $g$ germes $\mathcal{K}$ - $\mathcal{M}$-bi-Lipschitz cquivalentes. Então, existe um homeomorfismo bi-Lipschitz $M$ satisfazendo

$$
M\left(\mathbb{R}^{n} \times\{0\}\right)=\mathbb{R}^{n} \times\{0\} \text { e } M(\operatorname{graf}(f))=\operatorname{graf}(g) .
$$

Seja $h:\left(\mathbb{R}^{n}, 0\right) \rightarrow\left(\mathbb{R}^{n}, 0\right)$ definido por $h(x)=\pi_{n}(M(x, f(x)))$.

A firmação 1. hó um germe de aplicação bi-Lipschitz.

De fato, como $g$ é uma função Lipschitz, a projeção $\pi_{n_{\mid \text {graf }(g)}}$ é uma aplicação biLipschitz. Pelo mesmo argumento, a aplicação $x \mapsto(x, f(x))$ é bi-Lipschitz. Por definição, a aplicação de Mather-Montaldi $M$ é bi-Lipschit\%. Logo, a aplicação $h$ é bi-Lipschitz c a Afirmação 1 está provada.

Afirmação 2. Uma das seguintes condições é verdadeira:

i) $\int \approx g \circ h$,

ii) $f \approx-g \circ h$.

De fato, como $M$ é uma aplicação bi-Lipschitz, temos que existem constantes reais positivas $c_{1}$ e $c_{2}$, tais que

$$
c_{1}|f(x)| \leq\|M(x, f(x))-M(x, 0)\| \leq c_{2}|f(x)| .
$$

Mas, pela construção acima

$$
\|M(x, f(x)\}-M(x, 0)\|=\|(h(x), g(h(x)))-M(x, 0)\| \geq|g(h(x)\}| .
$$

Logo, $|g(h(x))| \leq c_{2}|f(x)|$.

Usando o mesmo procedimento, para a aplicação $M^{-1}$, obtemos

$$
c_{1}|f(x)| \leq|g(h(x))|
$$

Como $M$ é um homeomorfismo e $M\left(\mathbb{R}^{n} \times\{0\}\right)=\mathbb{R}^{n} \times\{0\}$, assim como no Teorema 6.9 podemos concluir que, para todo $x \in \mathbb{R}^{n}$,

$$
\operatorname{sinal}[f(x)]=\operatorname{sinal}[g(h(x))]
$$

ou, para todo $x \in \mathbb{R}^{n}$,

$$
\sin a l[f(x)]=\operatorname{sinal}[-g(h(x))] .
$$


Portanto, a Afirmação es está provada.

Finalizando a prova do 6.13.

Usando a Afirmação 2 obtemos, pelo Teorema 6.9, que $f$ e $g \circ h$ são $\mathcal{C}$-bi-Lipschit\% equivalentes. Pela Afirmação $1, f$ and $g$ são $\mathcal{K}$-bi-Lipschitz equivalentes.

\subsection{Teorema de finitude}

Lista seção é dedicada à prova da finitude dos tipos $\mathcal{K}$-bi-Lipschitz dos germes de funçõos polinomiais.

Teorema 6.14. Considere $\mathcal{P}_{k}\left(\mathbb{R}^{n}\right)$ o conjunto de todos os polinômios de $n$ variáveis com grau menor ou igual a $k$. Então o conjunto de classes de equivalência, com respeito a $\mathcal{K}$-bi-Lipschitz equivalência, dos germes em 0 dos polinômios em $\mathcal{P}_{k}\left(\mathbb{R}^{n}\right)$ é finito.

Prova. Scjam $f \in \mathcal{P}_{k}\left(\mathbb{R}^{n}\right)$ e $X_{f}$ um germe do conjunto $\mathbb{R}^{n} \times\{0\} \cup$ graf $(f)$. Pelo Teorema de Parusinski [47], existe uma estratificação scrnialgébrica finita $\left\{\mathcal{S}_{i}\right\}_{i=1}^{p(k)}$ de $\mathcal{P}_{k}\left(\mathbb{R}^{n}\right)$ satisfazendo as seguintes condições:

i) Cada $\mathcal{S}_{i}$ ć uma variedade semialgébrica conexa.

ii) Para cada $\mathcal{S}_{i}$ e para cada $f \in \mathcal{S}_{i}$, existe uma vizinhança $U_{f}$ tal que $U_{f} \Gamma_{i} \mathcal{S}_{i}$ é conexa por caminhos e para qualquer $\tilde{f} \in U_{f} \cap \mathcal{S}_{i}$ e para qualquer caminho $\gamma:[0,1] \rightarrow U_{f} \cap \mathcal{S}_{i}$ ligando $f$ a $\tilde{f}$ (isto é, $\gamma(0)=f$ e $\gamma(1)=\tilde{f}$ ), existe uma família de germes de aplicações bi-Lipschitz $\rho_{t}:\left(\mathbb{R}^{n} \times \mathbb{R}, 0\right) \rightarrow\left(\mathbb{R}^{n} \times \mathbb{R}, 0\right)$, onde $\rho_{0}=i d_{\mathbb{R}^{n} \times \mathbb{R}}$ e $\rho_{t}\left(X_{f}\right)=X_{\gamma(t)}$.

A condição ii) implica que, para pequenos valores de $t, \rho_{t}$ é uma aplicação de MatherMontaldi. De fato, podemos considerar a distância de Hausdorff para garantir que localmente, $\rho_{t}\left(\mathbb{R}^{n} \times\{0\}\right)=\left(\mathbb{R}^{n} \times\{0\}\right)$ e $\rho_{t}(\operatorname{graf}(f))=\operatorname{graf}(\bar{f})$, onde $\bar{f}=\gamma(t)$, para algum $t$ pequeno.

Mas, como $\mathcal{S}_{i}$ é um conjunto conexo, então esta propriedade é global, ou seja, para quaisquer dois polinômios $f_{1}, f_{2} \in \mathcal{S}_{i}$, existe uma aplicação de Mather-Montaldi $M:\left(\mathbb{R}^{n} \times \mathbb{R}, 0\right) \rightarrow\left(\mathbb{R}^{n} \times \mathbb{R}, 0\right)$ tal que $M\left(X_{f_{1}}\right)=X_{f_{2}}$.

Observe que qualquer classe de equivalência da $\mathcal{K}$ - $\mathcal{M}$-bi-Lipschit/ cquivalência cm $\mathcal{P}_{k}\left(\mathbb{R}^{n}\right)$ é uma união finita de alguns estratos da estratificação $\left\{\mathcal{S}_{i}\right\}_{i=1}^{p(k)}$. Pelo Teorema 6.13 , o conjunto das classes de equivalência com respcito à $\mathcal{K}$-bi-Lipschitz equivalência é também finito. 


\subsection{Resultados adicionais}

Dadas as funções $f, g: \mathbb{R}^{n} \rightarrow \mathbb{R}$, denotamos por $f \simeq g$ quando existe uma vizinhança $U$ da origem em $\mathbb{R}^{n}$ e constantes reais positivas $c_{1}$ e $c_{2}$ tais que

$$
c_{1}|f(x)| \leq|g(x)| \leq c_{2}|f(x)|, \quad \forall x \in U
$$

Em particular, se os germes de funções $f$ e $g$ satisfazem $f \simeq g$ então os germes dos conjuntos de zero coincidem em $U$, isto é, $f^{-1}(0)=g^{-1}(0)$ nà vizinhança $U$.

Para germes $\mathcal{C}^{0}-\mathcal{K}$-finitos, podemos obter um resultado análogo ao Teorema 6.9 , substituindo $\approx$ por $\simeq$, do seguinte modo:

Proposição 6.15. Sejam $f, g:\left(\mathbb{R}^{n}, 0\right) \rightarrow(\mathbb{R}, 0), n \geq 2$, germes de funções Lipschitz, $\mathcal{C}^{0}-\mathcal{K}$-finitamente determinadas. Então, $f$ eg são $\mathcal{C}$-bi-Lipschitz equivalentes se, e somente se, $f \simeq g$.

Prova. De fato, se $f$ e $g$ são $\mathcal{C}$-bi-Lipschiť equivalentes, então $f \simeq g$, pelos mesmos argumentos usados no Teorema 6.9. Mais ainda, como já vimos $f \approx g$.

Reciprocamente, se $f \simeq g$, existe uma vizinhança $\bar{U}$ de 0 em $\mathbb{R}^{n}$ tal que $f^{-1}(0)=g^{-1}(0)$ cm $\bar{U}$. Como $f$ (resp. $g$ ) è $\mathcal{C}^{0}-\mathcal{K}$-finito, existe uma vizinhança $U^{\prime}$ da origem em $\mathbb{R}^{n}$ tal que qualquer ponto de $f^{-1}(0) \cap U^{\prime}-\{0\}$ (resp. $g^{-1}(0) \cap U^{\prime}-\{0\}$ ) é ponto regular. Seja $U=\vec{U} \cap U^{\prime}$. Então, como germes na origem, uma das seguintes condições acontece:

a) $\operatorname{sinal}[f(x)]=\operatorname{sinal}[g(x)], \quad \forall x \in U-f^{-1}(0)$, ou

b) sinal $[f(x)]=\operatorname{sinal}[-g(x)], \quad \forall x \in U-f^{-1}(0)$.

Suponha que a) ocorra. Então, $f \simeq g$ implica $f \approx g$ e pelo Teorema 6.9 o resultado seguc. Concluímos o mesmo se b) ocorre.

Definição 6.16. Seja $h:\left(\mathbb{R}^{n}, 0\right) \rightarrow\left(\mathbb{R}^{n}, 0\right)$ um germe de homeomorfismo bi-Lipschitz. Definimos $f \stackrel{h}{\simeq} g$ quando $f \simeq g \circ h$.

Proposição 6.17. Sejam $f, g:\left(\mathbb{R}^{n}, 0\right) \rightarrow(\mathbb{R}, 0)$ germes de funções Lipschitz. Então, $f$ e $g$ são $\mathcal{K}$-bi-Lipschitz equivalentes se, e somente se, $f \stackrel{h}{\simeq} g$ e $f^{\mathcal{C}^{0}-\mathcal{K}} \sim$.

Prova. Suponha $f$ e $g \mathcal{K}$-bi-Lipschitz equivalentes. Então, existem homeomorfismos bi-Lipschitz: $H:\left(\mathbb{R}^{n} \times \mathbb{R}, 0\right) \rightarrow\left(\mathbb{R}^{n} \times \mathbb{R}, 0\right)$ e $h:\left(\mathbb{R}^{n}, 0\right) \rightarrow\left(\mathbb{R}^{n}, 0\right)$ tais que

$$
H(x, 0)=(h(x), 0) \text { e } H(x, f(x))=(h(x), g(h(x)))
$$


Então,

$$
|g(h(x))|=\|(h(x), 0)-(h(x), g(h(x)))\|=\|H(x, 0)-H(x, f(x))\| \leq c_{1}|f(x)| .
$$

Procedendo de modo análogo com a inversa de $H$ obtemos $c_{2}|f(x)| \leq|g(h(x))|, c_{2}>0$. Portanto, $f \simeq g \circ h$, ou seja, $f \stackrel{h}{\simeq} g$. Além disso, é claro que se $f$ e $g$ são $\mathcal{K}$-bi-Lipschitz equivalentess entĩo estes germes são $\mathcal{C}^{0}-\mathcal{K}$-equivalentes.

Reciprocarrıente, se $f \stackrel{\mathcal{C}^{0} \mathcal{X}}{\sim} g$ : temos que existe uma vizinhança $U$ da origem em $\mathbb{R}^{n}$ tal que $f^{-1}(0)=(g \circ h)^{-1}(0)$ em $U$. Além disso, como já vimos anteriormente, temos que os sinais de $f$ e $g \circ h$ ou coincidem nas componentes conexas de $U-f^{-1}(0)$ ou são trocados. Suponha que uma das duas situações aconteça. Usando este fato e a hipótese $f \stackrel{h}{\simeq} g$, isso implica que $f \approx g \circ h$. Portanto, pelo Tcorema $6.9, f$ e $g \circ h$ são $\mathcal{C}$-bi-Lipschitz equivalentes, e entã̃o, $f$ e $g$ são $\mathcal{K}$-bi-Lipschitz equivalentes.

O resultado acima mostra que a $\mathcal{C}^{0}-\mathcal{K}$-equivalência e a condição $\stackrel{h}{\simeq}$ formam um sistema completo de invariantes para a $\mathcal{K}$-bi-Lipschitz equivalência dos germes de funções.

\subsection{Outros invariantes da $\mathcal{K}$-bi-Lipschitz equivalência}

Seja $X \subset \mathbb{R}^{n}$ um conjunto semialgébrico conexo. Podemos considerar $X$ um espaço métrico com respeito a duas diferentes métricas: a cuclidiana $\left(\mathrm{d}_{e}\left(x_{1}, x_{2}\right)=\| x_{1}-x_{2}||\right)$ e a intrínsica $\left(\mathrm{d}_{\text {int }}\left(x_{1}, x_{2}\right)=\inf \left\{|\gamma| \mid \gamma\right.\right.$ é um caminho ligando $x_{1}$ a $\left.x_{2}\right\}$.)

Proposição 6.18. (Lojasiewicz [30]) Existe um número racional $0<\alpha \leq 1$ e um número real $k>0$ tal que para cada $x_{1}, x_{2} \in X$ temos

$$
\mathrm{d}_{\text {int }}\left(x_{1}, x_{2}\right) \leq k\left\|x_{1}-x_{2}\right\|^{\alpha} .
$$

O número $\alpha_{0}$ definido como o máximo de tais números racionais $\alpha$ é chamado expoente de Lojasiewicz de $X$.

Proposição 6.19. O expoente de Lojasiewicz é um invariante da $\mathcal{K}$-bi-Lipschitz equivalência.

Prova. A idéia da prova é a mesma usada cm [8], p. 27.

Sejam $f, g:\left(\mathbb{R}^{n}, 0\right) \rightarrow(\mathbb{R}, 0)$ germes $\mathcal{K}$-bi-Lipschitz cquivalentes. Então, pelo Teorema 6.13, $f$ e $g$ são $\mathcal{K}$-M-bi-Lipschitz equivalentes. Considere $X=\mathbb{R}^{n} \times\{0\} \cup \operatorname{graf}(f)$ 
e $Y=\mathbb{R}^{n} \times\{0\} \cup \operatorname{graf}(g)$. Logo, existe um homeomorfismo bi-Lipschitz $M$ tal que $M(X)=Y$. Então, existe $k_{1}>0$ tal que para cada $y_{1}, y_{2} \in Y$,

$$
\mathrm{d}_{\text {int }}\left(y_{1}, y_{2}\right) \leq k_{1} \mathrm{~d}_{\text {int }}\left(M^{-1}\left(y_{1}\right), M^{-1}\left(y_{2}\right)\right) .
$$

Mas, por (6.2),

$$
\mathrm{d}_{i n t}\left(M^{-1}\left(y_{1}\right), M^{-1}\left(y_{2}\right)\right) \leq k\left\|M^{-1}\left(y_{1}\right)-M^{-1}\left(y_{2}\right)\right\|^{\alpha_{0}(X)} .
$$

Como $M$ é bi-Lipschitz, existe $k_{2}>0$ tal que

$$
\left\|M^{-1}\left(y_{1}\right)-M^{-1}\left(y_{2}\right)\right\| \leq k_{2}\left\|y_{1}-y_{2}\right\|
$$

Então,

$$
\mathrm{d}_{i n t}\left(y_{1}, y_{2}\right) \leq k_{1} k k_{2}^{\alpha_{0}(X)}|| y_{1}-y_{2} \|^{\alpha_{0}(X)} .
$$

Portanto, $\alpha_{0}(Y) \geq \alpha_{0}(X)$. Considerando agora a inversa $M^{-1}$ de $M$, obtemos

$$
\alpha_{0}(X) \geq \alpha_{0}(Y)
$$

Logo, $\alpha_{0}(X)=\alpha_{0}(Y)$, como queríamos.

Pelo Tcorema 6.9, se $f \mathrm{e} g$ são germes de funções analíticas, $\mathcal{C}$-bi-Lipschitz equivalentes, então $f \approx g$ c, portanto, eles têm a mesma multiplicidadc. Fntretanto, a multiplicidade não é um invariante completo para a $\mathcal{C}$-bi-Lipschit» equivalência. De fato, os germes

$$
f(x, y)=x^{2}+y^{2} \text { e } g(x, y)=x^{2}+y^{4}
$$

têm a mesma multiplicidade 2 , mas não têm a mesma ordem, ou seja, $f \approx g$ não ocorre.

A proposição a seguir mostra um caso particular onde a multiplicidade torna-se um invariante completo da $\mathcal{C}$-bi-Lipschitz equivalência.

Proposição 6.20. Sejam $f, g:\left(\mathbb{R}^{n}, 0\right) \rightarrow(\mathbb{R}, 0)$ germes de funções analíticas tais que $f^{-1}(0)=g^{-1}(0)=\{0\}$. Sejam $X=\mathbb{R}^{n} \times\{0\} \cup \operatorname{graf}(f)$ e $Y=\mathbb{R}^{n} \times\{0\} \cup \operatorname{graf}(g)$. Suponha que

$$
m_{f}=m_{y}=\alpha_{0}(X)=\alpha_{0}(Y)
$$

Então, $f$ éC-bi-Lipschitz equivalente a $g$.

Prova. Observe que para qualquer curva $\gamma:[0,1] \rightarrow \mathbb{R}^{n}$, temos que

$$
f(\gamma(t)) \lesssim t^{m_{f}}=t^{\alpha_{0}(X)} \lesssim f(\gamma(t)) .
$$

Logo, $t^{m_{f}} \approx f(\gamma(t))$. Idem para $g$. Mas, $t^{m_{f}}=t^{m_{g}}$. Logo, $f \approx g$. Portanto, pelo Teorema $6.9, f$ c $\mathcal{C}$-bi-Lipschitz equivalente a $g$. 


\section{Comentários finais}

1) Conforme vimos na Proposição 1.45 e no Corolário 1.46, Mather [33] estabelece condições que caracterizam a $\mathcal{C}$ e a $\mathcal{K}$-equivalência. Com os resultados destc trabalho obtidos no Capitulo 4, para germes de funçöes reais $\mathcal{C}^{0}-\mathcal{K}$-equivalentes, e no Capítulo 6, para germes de funçõos reais $\mathcal{K}$-bi-Lipschitz equivalentes, estabelecemos as versões topológica e bi-Lipschit: dos resultados de Mather para o caso de funçōs.s. Observe os quadros a seguir:

\begin{tabular}{|c|c|c|c|}
\hline Caso diferenciável & $f \stackrel{\mathcal{C}}{\sim}_{g}$ & $\langle f\rangle=\langle g\rangle$ & $\exists M_{1 \times 1} \mid f=M \cdot g$ \\
\hline Caso bi-Lipschitz & $f \stackrel{\mathcal{C}-\text { bil }}{\sim} g$ & $\overline{\langle f\rangle_{R}}=\overline{\langle g\rangle_{\mathbb{R}}}$ & $f \approx g$ \\
\hline Caso topológico & $\int \stackrel{\mathcal{C}^{0} \mathcal{C}}{\sim} g$ & $\operatorname{sinal}(f)=\operatorname{sinal}(g)$ & $\exists \alpha, \beta$ tendas $\mid \alpha \stackrel{\mathcal{C}^{0} \sim \mathcal{C}}{\sim} f, \beta \stackrel{\mathcal{C}^{10}-\mathcal{C}}{\sim} g$ \\
\hline
\end{tabular}

\begin{tabular}{|c|c|c|c|}
\hline Caso difcrenciável & $f \stackrel{\mathcal{K}}{\sim} g$ & $\langle f\rangle=\langle g \circ h\rangle$ & $\exists M_{1 \times 1} \mid f=M \cdot g \circ h$ \\
\hline Caso bi-Lipschitz & $f^{\mathcal{K}} \stackrel{\text { bil }}{\sim} g$ & $\overline{\langle f} \overline{\rangle}_{\mathbb{R}}=\overline{\langle g \circ h\rangle}$ & $f \approx g \circ h$ \\
\hline Caso topológico & $f^{\mathcal{C}^{0}-\mathcal{K}} \sim_{g}$ & $\operatorname{sinal}(f)=\operatorname{sinal}(g \circ h)$ & $\exists \alpha, \beta$ tendas $\mid \alpha^{\mathcal{C}^{0} \sim \mathcal{C}} \sim, \beta^{\mathcal{C}^{0} \sim \mathcal{C}} \sim g \circ h$ \\
\hline
\end{tabular}

Além disso, juntando os resultados obtidos na tese com os resultados de MatherMontaldi, obtemos o seguinte resultado:

$$
\begin{gathered}
f \stackrel{G}{\sim} g \Leftrightarrow \exists \text { umr } G \text {-isomorfismo } H \text { tal que } \\
H\left(\mathbb{R}^{n} \times\{0\}\right)=\mathbb{R}^{n} \times\{0\} \text { e } I I(\operatorname{graf}(f))=\operatorname{graf}(g),
\end{gathered}
$$

onde $G$ representa a equivalência de contato nas versões $C^{\infty}$, bi-Lipschitz e topológica, respectivamente.

2) O estudo de germes do aplicaçốes com respeito a $\mathcal{A}$-equivalência é motivo de grande interesse em teoria de singularidades. L'm dos problemas interessantes é responder ao problema do reconhecimento, ou sçja, dizer quando dois germes são $\mathcal{A}$-equivalentes. Esta 
mesma pergunta ocorre no caso topológico, para a $\mathcal{C}^{0}$-A-equivalência. Nesta linha de pesquisa, Nishimura [44], [46] propõe um método de construir a $\mathcal{A}$-cquivalência ou a $\mathcal{C}^{0}-\mathcal{A}$ equivalência para dois germes, a partir de uma $\mathcal{K}$-equivalência entre eles. Isso mais uma vez ressalta a importância da $\mathcal{K}$-equivalência, e é um tipo de argumento bastante usado pelos autores que buscam classificar $\mathcal{A}$-órbitas. Observe que a $\mathcal{A}$-classificação apresenta $\mathcal{C}^{\infty}$ moduli e então, dentro de uma $\mathcal{K}$-órbita existem infinitas $\mathcal{A}$-órbitas.

3) As direções que pretendemos seguir num estudo futuro são as seguintes:

i) obter melhores resultados para a $\mathcal{C}^{0}-\mathcal{K}$-equivalência dos germes de aplicações com $n>p$, encontrando possíveis invariantes, e estabelecendo um resultado geométrico análogo aos propostos para germes de funções;

ii) aprofundar o estudo dos germes de funções bi-Lipschitz, tentando encontrar outros invariantes, formas normais e, iniciar o estudo do caso de germes de aplicaçôes;

iii) a partir do nosso resultado de finitude para os tipos $\mathcal{K}$-bi-Lipchitz dos germes de funções, investigar o que acontece no caso dos germes de aplicações, ou seja, será que para germes de aplicações polinomiais existe um número finito de $\mathcal{X}$-bi-Lipchitz classes de equivalência?

iv) motivados pelo que acontece no caso $C^{\infty}$ (Teoremas 1.43 e 1.44 ), verificar se esta mesma abordagem se verifica para o caso bi-Lipschitz.

v) sabendo que, o conjunto das aplicaçõos $C^{0}$-estáveis é denso no conjunto

$$
C_{p r}^{\infty}\left(\mathbb{R}^{n}, \mathbb{R}^{p}\right)=\left\{f:\left(\mathbb{R}^{n}, 0\right) \rightarrow\left(\mathbb{R}^{p}, 0\right) \mid f \text { é própria }\right\}
$$

e que as aplicaçōes $C^{1}$-estáveis não são densas, uma pergunta natural é a seguinte:

Fora das boas dimensões de Mather, o conjunto das aplicações bi-Lipschitz estáveis é denso em $C_{p r}^{\infty}\left(\mathbb{R}^{n}, \mathbb{R}^{p}\right)$ ?

Resolver esta questão significa completar o estudo da estabilidade para os germes de aplicações diferenciáveis. 


\section{Referências Bibliográficas}

[1] S. Alvarez, L. Birbrair, J.C.F. Costa, A.C.G. Finandes, Topological K-equivalence of analytic function-germs, submetido para publicação (2005).

[2] K. Aoki, T. Fukuda, T. Nishimura, On the number of branches of the zero locus of a map-germ $\left(\mathbb{R}^{n}, 0\right) \rightarrow\left(\mathbb{R}^{n-1}, 0\right)$, Topology and Computer Science, S. Suzuki ed. Kinokuniya Co. Ltd. Tokyo (1987) 347-363.

[3] K. Bekka, C-régulurité et trivialité topologique (French) [C-regularity and topological triviality] Singularity theory and its applications, Part I (Coventry, 1988/1989), Lecture Notes in Math. 1462, Springer, Berlin (1991) 42-62.

[4] K. Bokka, S. Koikc, Kuo condition, an inequality of Thom's type and (c)-regularity, 'lopology 37 (1998), no. 1, 45-62.

[5] R. Benedetti, J.J. Risler, Real algebraic and semialyebraic sets, Actualités Mathématiques, Hermann, Éditeurs Des Sciences et des Arts (1990).

[6] R. Benedetti, M. Shiota, Finiteness of semialgebraic types of polynomial functions, Math. Z. 208 (1991), no. 4, 589-596.

17] L. Birbrair, Local bi-Lipschitz classification of 2-dimensional semialgebraic sets, Houston J. Math. 25 (1999), no. 3, 453-472.

[8] L. Birbrair, F. Cano, Characteristic expoents of semialgebraic singularities, Math. Nachr. 276 (2004) 23-30.

[9] L. Birbrair, J.C.F. Costa, A.C.G. Fernandes, M.A.S. Ruas, Bi-Lipschitz Kequivalence of function-germs, aceito para publicação no Proc. Amer. Math. Soc. (2005).

[10] J. Bochnak, Relèvement des jets, Lecture Notes in Math. 275, Springer, Berlin (1972) 106-118. 
[11] J. Bochnak, M. Coste, M.-F. Roy, Real algebruic geometry, Springer-Verlag, Berlin (1998).

[12] J.W. Bruce, M.A.S. Ruas, M.J. Saia, A Note on Determinacy, Proc. Amer. Math. Soc. (1992), no. 3, 865-887.

[13] M. Coste, Topological types of fewnomials, Singularities Symposium-Lojasiewicz 70 (Kraków, 1996; Warsaw 1996), Banach Center Publ. 44, Polish Acad. Sci., Warsaw (1998) 81-92.

[14] M. Coste, An introduction to o-minimal geometry, Dottorato di Ricerca in Matematica, Dip. Mat. Univ. Pisa, Instituti Editoriali e Poligrafici Internazionali (2000).

[15] L. van den Dries, Tame topology and o-minimal structures, London Math. Soc., Lecture Note Series 248, Cambridge University Press, Cambridge (1998).

[16] D. Eisenbud, H.I Levine, An algebraic formula for the degree of a $\mathcal{C}^{\infty}$ map-germ, Ann. Math. 106 (1977) 19-44.

[17] A.C.G. Fernandes, M.A.S. Ruas, Bilipschitz determinacy of quasihomogeneous germs, Glasgow Math. J. 46 (2004), no. 1, 77-82.

[18] T. Fukuda, Types topologiques des polynômes, Publ. Math. I.E.H.S. 46 (1976) 87-106.

[19] T. Fukuda, Local topological properties of differentiable mappings I, Inventiones Math. 65 (1981), no. 2, 227-250.

$[20]$ A. Gabrielov, Complements of subanalytic sets and existential formulas for analytic: functions, Inventiones Math. 125 (1996), no. 1, 1-12.

[21] 'T. Gaffney, Properties of finitely determined germs, Ph.D. Thesis, Brandcis University (1975).

[22] T. Gaffney, Integral closure of modules and Whitney equisingularity, Inventiones Math. 107 (1992), no. 2, 301-322.

[23] C.G. Gibson, Singular points of smooth mappings, Research Notes in Math. 25, Pitman, London (1979).

[24] C.G. Gibson, E.J.N. Looijenga, A.A. du Plesis, K. Wirthmuller, Topological stability of smooth mappings, Lecture Notes in Math. 552, Springer-Verlag, Berlin-New York (1976). 
[25] J.-P. Henry, A. Parusinski, Existence of moduli for bi-Lipschitz equivalence of analytic function-germs, Compositio Math. 136 (2003), no. 2, 217-235.

[26] J.-P. Henry, A. Parusinski, Invariants of bi-Lipschitz equivalence of real analytic: functions, Geometric Singularity Theory, Banach Center P'ubl. 65, Polish Acad. Sci., Warsaw (2004) 67-75.

[27] H.C. King, Topological type in families of germs, Inventiones Math. 62 (1980) 1-13.

[28] T.C. Kuo, On $C^{0}$-sufficiency of jets of potential functions, Topology 8 (1969), $167-$ 171.

[29] S. Lojasiewicz, Triangulations of semi-analytic sets, Ann. Sc. Norm. Super. Pisa, Cl. Sci. (3) 18 (1964) 449-474.

[30] S. Lojasiewicz, Sur les ensembles semi-analytiques (French) Actes du Congrès International des Mathématiciens (Nice, 1970), Tome 2, 237-241.

[31] E.J.N. Loojenga, Isolated singular points on complete intersections, London Math. Soc., Lecture Note Series 77, Cambridge University Press, Cambridge (1984).

[32] J. Martinet, Déploiements versels des applications différentiables et classification des applications stables, Lecture Notes in Math. 535, Springer, Berlin (1976) 1-44.

[33| J. Mather, Stability of $\mathcal{C}^{\infty}$-mappings, III: finitely determined map-germs, Publ. Math. I.H.E.S. 35 (1969) 127-156.

[34] J. Mather, Stability of $\mathcal{C}^{\infty}$-mappings, IV: classification of stable map-germs by $\mathbb{R}$ algebras, Publ. Math. I.H.E.S. 37 (1970) 223-248.

[35] J. Mather, Stability of $C^{\infty}$ mappings, VI: the nice dimensions, Proc. of Liverpool Singularities-Symposium, I (1969/70) 207-253. Lecture Notes in Math. 192, Springer, Berlin (1971).

[36] J. Mather, Stratifications and mappings, Proc. Conference on Dynamical Systems, ed. by M. Peixoto, Academic Press (1973) 195-232.

[37] J.W. Milnor, Singular points of complex hypersurfaces, Annals of Math. Studies, no. 61, Princeton University Press (1968).

[38] D. Mond, On the classification of germs of maps from $\mathbb{R}^{2}$ to $\mathbb{R}^{3}$, Proc. London Math. Soc. (3) 50 (1985), no. 2, 333-369. 
|39| J. Montaldi, On contact between submanifolds, Michigan Malh. J. 33 (1986) 195-199.

|40| T. Mostowski, Topological equivalence between analytic and algebraic sets, Bull. Polish Acad. Sci. Math. 32 (1984), no. 7-8, 393-400.

[41] T. Mostowski, Lipschitz equisingularity, Dissertationes Math. 243 (1985).

[42] I. Nakai, On topological types of polynomial mappings, Topology 23 (1984), no. 1 , $45-66$.

[43] A.T.A. Nascimento, Distância Geodésica em conjuntos subunalíticos, Dissertação de Mestrado, LFC (2003).

|44| T. Nishimura, Topological types of finitely $\mathcal{C}^{0}-\mathcal{K}$-determined map-germs, Transactions of the Amer. Math. Soc., Vol 312, (1989), no. 2, 621-639.

|45| T. Nishimura, Topological $\mathcal{K}$-equivalence of smooth map-germs, Stratifications, Singularities and Differential Equations, I (Marseille 1990, Honolulu, Hll 1990), Travaux en Cours, 54, Hermann, Paris (1997) 82-93.

[46| T. Nishimura, Criteria for right-lefl equivalence of smooth map-germs, Topology 40 (2001), no. 3, 433-462.

[47| A. Parusinski, Lipschitz properties of semi-analytic sets, Ann. Inst. Fourier (Grenoble) 38 (1988), no. 4, 189-213.

[48] A. Parusinski, Lipschitz stratification of subanalytic sets, Ann. Sci. Ecole Norm. Sup. (4) 27 (1994), no. 6, 661-696.

|49| G. Perclman, Ricci Flow and Geometrization of Three-Manifolds, Massachusetts Institute of Technology Department of Mathematics Simons Lecture Series, http://www-math.mit.edu/conferences/simons;

The Entropy Formula for the Ricci Flow and Its Geometric Application (2002) htt.p://www.arxiv.org/abs/math.DG/0211159;

Ricci Flow with Surgery on Three-Manifolds (2003) http://www.arxiv.org/abs/math.DG/0303109

[50] J.-J. Risler, D. Trotman, Bi-Lipschitz invariance of the multiplicity, Bull. London Math. Soc. 29 (1997) 200-204. 
[51] M.A.S. Ruas, $C^{l}$-determinação finita e aplicações, Tese de Doutorado, ICMC-USP (1983).

[52| M.A.S. Ruas, On the degree of $C^{l}$-determinacy, Math. Scand. 59 (1986) 59-70.

[53] M.A.S. Ruas, J.M. Saia, $C^{l}$-determinacy of weighted homogeneous germs, Hokkaido Math. J. 26 (1997) 89-99.

[54] M.A.S. Ruas, R.N. dos Santos, Real Milnor fibrations and (c)-regularity, Manuscripta Math. 117 (2005), no. 2, 207-218.

[55] M.A.S. Ruas, J.N. Tomazella, Topological trimality of families of functions on analytic varieties, Nagoya Math. J. 175 (2004), 39-50.

[56] O. Sacki, Topological types of complex isolated hypersurface singularities, Kodai Math. J. 12 (1989) 23-29.

|57| M.J. Saia, Poliedros de equisingularidade de germes pré-quase-homogêneos, Tese de Doutorado, ICMC-USP (1991).

[58| C.H. Soares Jr, Poliedros de Neuton e trivialidade em famílias de aplicações, Tese de Doutorado, ICMC-USP (2003).

[59| Z. Szafranice, On the number of branches of an 1-dimensional semianalytic set, Kodai Math. J. 11 (1988) 78-85.

[60| B. Teissier, Multiplicities polaires, sections planes, et conditions de Whitney, Lectures Notes in Math. 961 (1982) 314-491.

[61| J.N. Tomazella, Seções de variedades analíticas, Tese de Doutorado, ICMC-USP (1999)

|62| J.C. Tougeron, Idéaux de fonctions différentiables I, Ann. Inst. Fourier 18 (1968) $177-240$.

[63| J.C. Tougeron, Idéaux de fonctions différentiables, Ergebnisse, Band 71, SpringerVerlag (1972).

[64] D. Trotman, Comparing regularity conditions on stratifications, Singularities, Part 2 (Arcata, Calif., 1981) Proc. Sympos. Pure Math., 40, Amer. Math. Soc., Providence, R.I. (1983) 575-586. 
[65] C.T.C. Wall, Finite determinacy of smooth map-germs, Bull. London Math. Soc. 13 (1981), no. 6, 481-539. 